\title{
Graphene and its derivatives: understanding the main chemical and medicinal chemistry roles for biomedical applications
}

\author{
Tais Monteiro Magne ${ }^{1} \cdot$ Thamires de Oliveira Vieira $^{1} \cdot$ Luciana Magalhães Rebelo Alencar $^{2}$. \\ Francisco Franciné Maia Junior ${ }^{3}$. Sara Gemini-Piperni ${ }^{4}$. Samuel V. Carneiro ${ }^{5}$. Lillian M. U. D. Fechine . $^{5}$ \\ Rafael M. Freire $^{6} \cdot$ Kirill Golokhvast $^{7,8} \cdot$ Pierangelo Metrangolo $^{9} \cdot$ Pierre B. A. Fechine $^{5} \cdot$ Ralph Santos-Oliveira $^{1,10}$ (i)
}

Received: 25 June 2021 / Accepted: 30 August 2021 / Published online: 6 September 2021

(C) Islamic Azad University 2021

\begin{abstract}
Over the past few years, there has been a growing potential use of graphene and its derivatives in several biomedical areas, such as drug delivery systems, biosensors, and imaging systems, especially for having excellent optical, electronic, thermal, and mechanical properties. Therefore, nanomaterials in the graphene family have shown promising results in several areas of science. The different physicochemical properties of graphene and its derivatives guide its biocompatibility and toxicity. Hence, further studies to explain the interactions of these nanomaterials with biological systems are fundamental. This review has shown the applicability of the graphene family in several biomedical modalities, with particular attention for cancer therapy and diagnosis, as a potent theranostic. This ability is derivative from the considerable number of forms that the graphene family can assume. The graphene-based materials biodistribution profile, clearance, toxicity, and cytotoxicity, interacting with biological systems, are discussed here, focusing on its synthesis methodology, physicochemical properties, and production quality. Despite the growing increase in the bioavailability and toxicity studies of graphene and its derivatives, there is still much to be unveiled to develop safe and effective formulations.
\end{abstract}

\section{Graphic abstract}

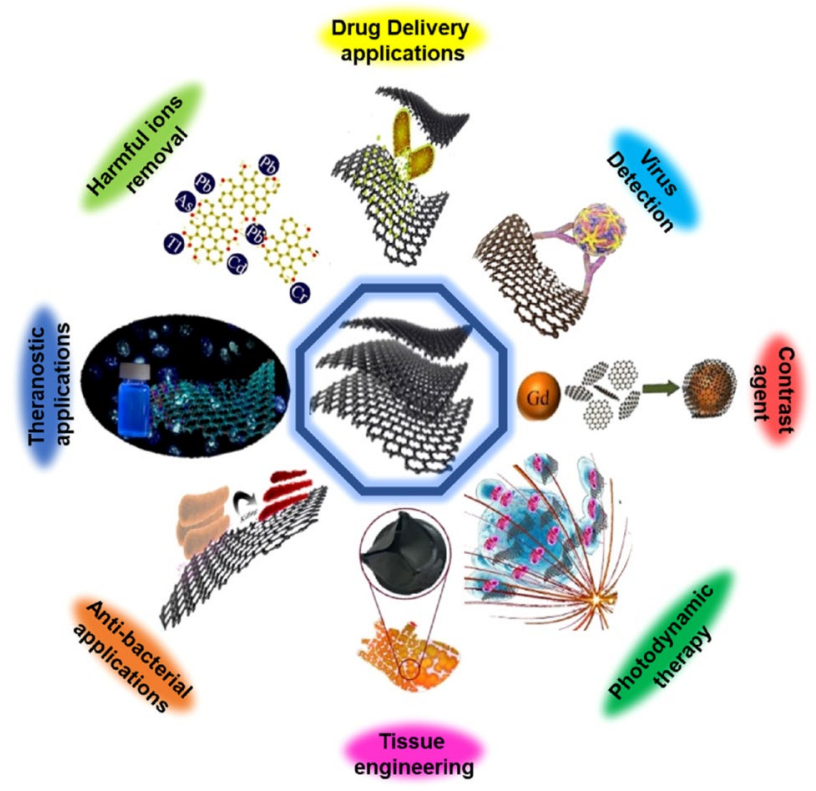

Keywords Graphene $\cdot$ Nanomaterials $\cdot$ Carbon $\cdot$ Nanoparticles $\cdot$ Nanomedicine

Extended author information available on the last page of the article 


\section{Introduction}

Advancement in nanotechnology and its applications to the field of medicines and pharmaceuticals have revolutionized the twentieth century. Nanotechnology studies small structures with a size range varying from 1 to $1000 \mathrm{~nm}$, and at least one dimension. Thus, nanotechnological-based materials have a broad range of applications and create various nanomaterials and nanodevices [1,2].

The application of nanotechnology in the medical field can assist in developing new means of diagnosis and treatment used in cancer, a disease whose early diagnosis is essential for the success of therapy. In this sense, particles of an organic nature (carbon-based nanoparticles, polymers, micelles, or lipids) or inorganic (semiconductor, noble metal, or magnetic nanoparticles) may be used for both diagnosis and cancer therapy [3-8]. Nanomedicine is one of the most promising nanotechnology applications in medicine and is identified as an essential strategy for diagnosis and cancer therapy. Applying nanoparticles allows the diffusion of drugs more quickly in the body and directs the drug to the specific target [9].

Carbon-based compounds have significant applications in biomedicine (Fig. 1). Among the carbon-based nanosystems, graphene and its derivative have increased carbon-based compound's employment in the biomedical area because of its unique structure and properties. Recently, Serrano-Aroca et al. [10] have proposed to use carbon-based nanomaterial against COVID-19. Regarding the chemistry of graphene and its derivative, the existence of functional groups, such as carboxylic acid $(\mathrm{COOH})$, hydroxyls $(\mathrm{OH})$, and epoxides (COC), on its surface, especially in graphene oxide (GO) and reduced graphene oxide (rGO), allows graphene to be coupled to different biomolecules, expanding the diversity of its biomedical applications [11-14].

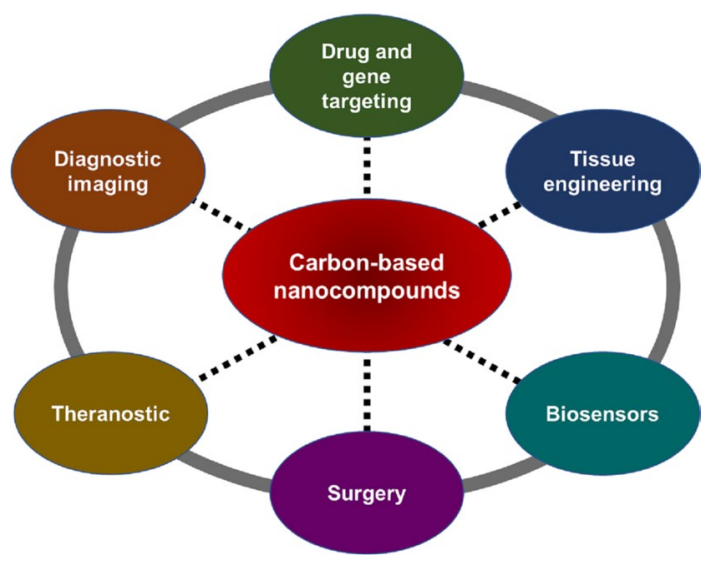

Fig. 1 Possible applications of carbon-based nanocompounds in the biomedical field. Source: authors
GO was first produced in 1860 by Brodie using graphite, potassium chloride, and nitric acid [15, 16]. Staudenmaier later introduced changes in the method that led to an increase in the oxidation process. Small amounts of chloride were added throughout the reaction, and the acidity increased by adding sulfuric acid [17]. The most used method for graphite oxide preparation is the Hummers method, developed in 1958 , and uses potassium permanganate $\left(\mathrm{KMnO}_{4}\right)$, sodium nitrate $\left(\mathrm{NaNO}_{3}\right)$, and sulfuric acid $\left(\mathrm{H}_{2} \mathrm{SO}_{4}\right)$ [18]. Sulfuric acid aids graphite oxidation with $\mathrm{KMnO}_{4}$, as it acidifies the $\mathrm{pH}$. Also, the $\mathrm{Mn}_{2} \mathrm{O}_{7}$ formed by the reaction of sulfuric acid and $\mathrm{KMnO}_{4}$ possesses strong oxidation ability, which plays a crucial role in forming graphene oxide. Hammer's method is adapted from Brodie's graphite oxide synthesis. In Brodie's methodology, potassium chlorate is added to graphite slurry in fuming nitric acid [19, 20]. Additionally, it adds alcohol, carboxylic acid, and deep oxide groups to the graphite molecule [21].

GO has been used in studies with prostate cancer [22], glioma [23], lung cancer [24], and esophageal cancer [25]. Additionally, it can be used as a carrier for drugs such as doxorubicin (DOX) [26], 5-fluorouracil [27], docetaxel [28], among other chemotherapies. It can also be applied in different techniques for the treatment and diagnosis of cancer. For instance, to selectively target cancer stem cells [29]. Finally, the use of graphene sheets showed to be efficient against SARS-Cov- 2 by direct interaction with cell receptors as surface proteins [30].

This review highlights the research conducted with graphene and its derivative and its use in forming conjugates with molecules of different classes applied in developing techniques for the therapy and diagnosis. The results show that biosensors, imaging examinations, and nano drugs can be produced by conjugating graphene and its derivative with other compounds, including metals, biomolecules, polymers, and many others.

\section{Review}

\section{Carbon-based nanomaterials}

Carbon-based nanomaterials are constituted of carbon atoms. These carbon atoms have covalent bonds composed of $\mathrm{sp}^{2}$ orbitals with a hexagonal arrangement, which originates from three types of nanostructures (1) zero-dimensional fullerenes and carbon quantum dots; (2) one-dimensional, carbon nanotubes; (3) and two-dimensional, graphene [31]. There are countless applications of this class of nanomaterials [32], including targeting drugs [33], biosensors [34-36], photoeletrochemisty [37], energy storage [38], and tissue engineering [39]. Also, carbon-based nanomaterials have demonstrated efficient antibacterial, antifungal, and antiviral 
properties. According to Rivera-Briso et al. [40], carbonbased nanomaterial showed relevant antibacterial activity avoiding biofilm formation. Carbon-based nanomaterial has demonstrated an important use as an antibacterial nanoagent, especially against Staphylococcus aureus and methicillin-resistant Staphylococcus epidermidis infections [41, 42], multidrug-resistant Staphylococcus epidermidis [43], and Gram-Positive Multidrug-Resistant Pathogens [44].

An important aspect related to carbon-based nanomaterial is the potential to enhance the biological properties of polymers. In this direction, Serrano-Aroca et al. [45] have crosslinked alginate films with GO to increase water perfusion by compressing this nanomaterial. Also, alginate-graphene oxide has been used to stabilize hydrogels [46-48] and increase biomedical applications [49-51].

\section{Graphene and its derivatives}

Graphene, the mother of all graphitic forms of carbon, consists of a crystalline material with $\mathrm{sp}^{2}$ hybridized carbon atoms, forming a hexagonal arrangement with a structure similar to honeycombs [52]. Graphene possesses an atomic thickness, which allows fluid permeability. The graphene honeycomb shape also enables its application for size-selective transport through its 2D nanochannels between adjacent stacked graphene sheets [53]. Due to all these features, graphene is considered a new building block for membranes [54], able to carry out effective sieving comparable to conventional materials.

Graphene is a recent addition to carbon allotropes and must outperform all other, becoming a helpful material for life and materials sciences due to its many intrinsic properties [55]. Because it has a very flexible structure, graphene can take different forms, such as sphere, ellipsoid, or even tubular (fullerene), cylinder [carbon nanotube (NTC)], or "stacked" graphene sheets (graphite). Bhuyan et al. reported that this material was made by two English scientists, Andre Geim and Konstantin Novoselov, at the University of Manchester, through an exfoliation from graphite giving the Nobel prize in physics to both [56]. Torres reported the graphene family had drawn the attention of an increasing number of researchers working in various areas from composites to molecular electronics [57]. The ability of graphene and its derivative to interact with other molecules through several processes that may include physical [58] and chemical [59] processes is one of these materials' main advantages.

The basic building blocks of all carbon nanostructures are a single graphical layer functionally $\mathrm{C}-\mathrm{C}$ carbon atoms linked in a hexagonal honeycomb structure that forms 3D bulk graphite when the layers of graphical honeycomb trusses are stacked and connected by a weak Van der Waals force. A graphite layer is monoatomic or single-layer graphene, while two to three graphite layers are bilayer and tri-layer graphene, respectively [56].

An interesting derivative of graphene is its reduced form rGO (reduced graphene oxide). rGO presents the formation of the layered deposit, accompanied by the removal of the oxygenated groups of graphene oxide (GO) through electrochemical oxidation, where the carboxylic acids pass in the form of $\mathrm{CO}_{2}$, producing a film of rGO, having a much lesser number of oxygen functional groups than GO [60]. Another example has been reported by Palanisamy et al. [61]. In their study using an rGO modified electrode, $8 \mu \mathrm{L}$ of GO solution was dropped on pre-cleaned GCE and dried in an air oven. Then, GO composite modified electrode was transferred into an electrochemical cell containing $\mathrm{pH} 5$ and applied a constant potential of $-1.4 \mathrm{~V}$ for $300 \mathrm{~s}$. Finally, GO was transformed into rGO. The rGO can be obtained from chemical, thermal, microwave, or photochemical methods, reducing its oxygen content [62]. Thus, it is expected that on the surface of rGO, there are eventually hydroxyl and epoxide groups, with only a partial reduction of GO. However, the main differences between $\mathrm{GO}$ and $\mathrm{rGO}$ are the predominance of carboxyl's, many defects, and lower oxygen content in the reduced form [63]. Recently, Ansari and Siddiqui [64] have used Ficus carica, to promote the deoxygenation of graphene oxide with good results (Fig. 2).

A growing area of science is the use of rGO in different polymeric materials. The interest stems from the possibility of preparing conductive composites with tailored electrical characteristics, possibly using the high inherent mechanical properties related to strong and rigid $\mathrm{sp}^{2}$ hybridized

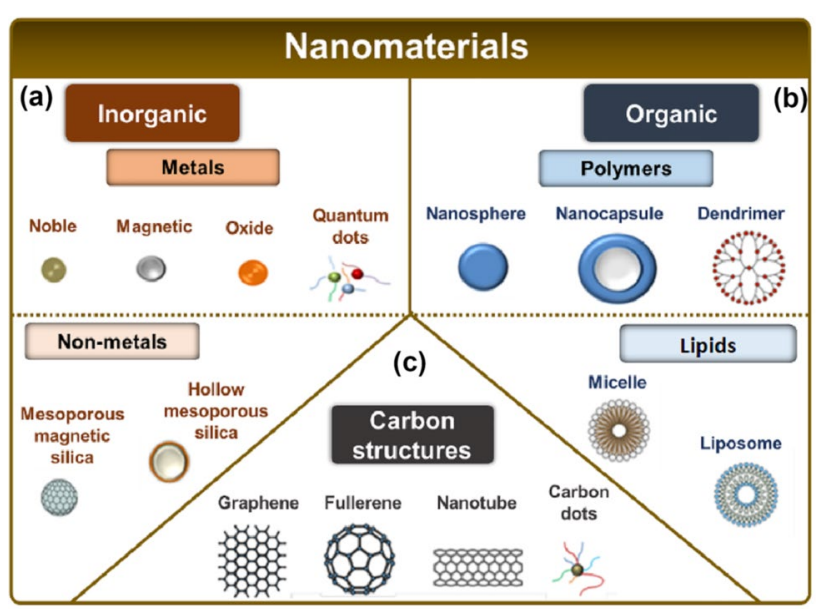

Fig. 2 Classification of nanomaterials according to their classes: a Inorganic nanomaterials, consisting of metallic (noble, magnetic, oxide, and quantum dots) and non-metallic (mesoporous silica with magnetic core and mesoporous silica hollow) nanomaterials; b organic nanomaterials, consisting of polymers (nanosphere, nanocapsules, and dendrimers) and lipids (micelles and liposome); and c carbon nanomaterials, consisting of graphene, fullerene, nanotubes, and carbon dots. Source: authors 
interconnected carbon atoms. Most studies on the applied use of rGO rely on the Hummers method used to exfoliate GO sheets and from graphite [65]. Graphite is the most well-known raw material used in the top-down approach to synthesize reduced rGO, while graphite oxide is obtained by treating the graphite with strong oxidizing agents. This material can be defined as a set of functionalized sheets of graphene formed by different functional oxygen groups, such as epoxides, hydroxides, and carboxyl. According to the report of Lavin-Lopez et al.[66], the incorporation of oxygen groups in graphite makes its structure less hydrophobic, allowing the separation of its layers in an aqueous solution by sonication to obtain GO [66]. Also, the presence of oxygen groups in $\mathrm{GO}$ and $\mathrm{rGO}$ has demonstrated increased hydrophilicity [67].

The method presents a vigorous laboratory route for the raw material that is beneficial because of the natural dispersion of the leaves resulting from the polarity of the surface introduced, which facilitates the separation of the leaves in the aqueous medium [65].

GO is another derivative of graphene considered one of the most visible results of graphene research in terms of prospects for large-scale production and commercialization. GO can be easily prepared by oxidation-exfoliation of graphite in solutions under stirring [68]. It is a two-dimensional (2D) crystalline structure made of carbon and organized in a hexagonal pattern with several functional oxygen groups on the surface. GO is considered a stronger tempering element with great potential in biodetection applications because of $\mathrm{sp}^{2}$ hybridization, $\pi-\pi$ and/or $n-\pi$ orbital interactions, and its versatile bioconjugation chemicals [69]. The functional groups of GO can be easily combined with other biomolecules and biomaterials. Additionally, the advantage of GO, compared to graphene, is its ease of dissolution in water and other solvents due to the functional groups of oxygen [70].

In the GO synthesis process, graphite oxide is first prepared by forming hydroxyl or carboxyl groups, covalently bound to a graphite planar carbon network. The material then is treating with oxidizing agents, such as sulfuric and nitric acid exfoliated in GO single-layer or monolayer containing a high density of deformations in its structure. Due to its capability to form a stable suspension in water, $\mathrm{GO}$ is one of the main product segments in the graphene market. Figure 3 shows graphene sheets of small size can be obtained by subsequent reduction of GO, which can eliminate most of its oxygen-containing functional groups and partially recover its carbon network linked to $\mathrm{sp}^{2}$ [71]. Thus, to conclude, graphene is a single carbon layer of graphite structure, describing its nature by the analogy of a polycyclic aromatic hydrocarbon of quasi-infinite size. The $\mathrm{rGO}$ is the preparation obtained from reduced graphene oxide by thermal, chemical, or electrical treatments.
Pristine graphene is another derivative of graphene studied, whose surface lacks chemical substances functionality to allow covalent modifications without interrupting its orbital $\pi$-system [72]. Taniselass et al. reported that the reduction of GO is the widely accepted route because this strategy can achieve pristine graphene [73]. The ease of production of pristine graphene, through the liquid phase method, allows us to generate the first high content of graphene bio-hybrids, which are commercially viable and exhibit superior biocompatibility [74].

The graphene has another origin called graphene quantum dots (GQDs), a quantum form of this material with small sizes (preferable less than $10 \mathrm{~nm}$ ), containing some layers of the material. This type of graphene may show high optical absorption in the UV region, enhancing electroluminescence [75]. The GQDs have been widely studied in recent years due to their unique structure-related properties, such as optical and electrical. Due to intrinsic inert carbon property and chemically and physically stable GQDs, they are considered a new class of quantum dots. Additionally, they are environmentally friendly due to their non-toxic properties and inertia in the biological environment and have attracted worldwide interest from the academic and industry sectors [76, 77].

In this regard, GQDs are nanomaterials exploited for medical applications have shown excellent performances, which could replace some traditional materials in this field. There have been continuous efforts in controlling these materials for other new medical applications due to recent promising results in the area [78]. Among these applications, we can mention electrochemical immunosensors [79], bioimaging [80], and drug delivery [81].

GQDs present several new nanomaterial features, including quantum confinement, size, and edge effects. Its advantages, such as low toxicity, water-solubility, high biocompatibility, and ease of functionalization, are crucial for biomedical applications. They have also attracted growing interests in catalysis, bioimage, and flowering detection [82].

Due to versatility, ranging from compounds to flexible electronics, several scientists have a growing interest in different fields. In biomedical applications, graphene is involved in the administration and targeting of drugs, genes, imaging agents, biosensors, and tissue engineering, with contributions to nanomedicine. With exciting results and success attained, there are still problems regarding the manufacture of biomedical devices, including the lack of standardization in the production of the members of the graphene family requiring attention [83]. Finally, it is essential to notice that GO nanosheets can be crosslinked by coordination chemistry with divalent cations of $\mathrm{Ca}, \mathrm{Zn}$, and $\mathrm{Sr}$ to form more extensive materials in the form of irregular tubes of GO [84]. 
Fig. 3 a Graphene oxide obtained by graphite exfoliation. b Is presented the reduced graphene oxide structured; as the graphene oxide defect. c Reactive groups present in graphene oxide and reduced graphene oxide. Source: authors (a)
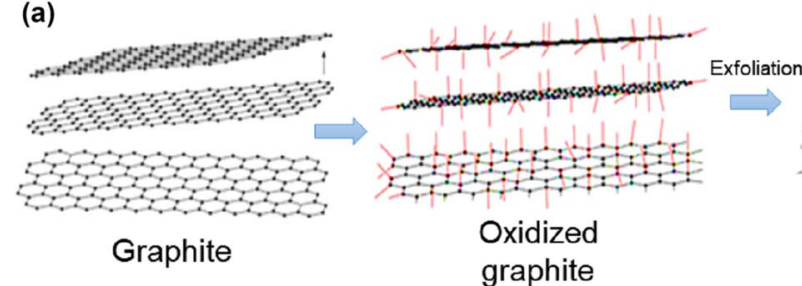

Oxidized

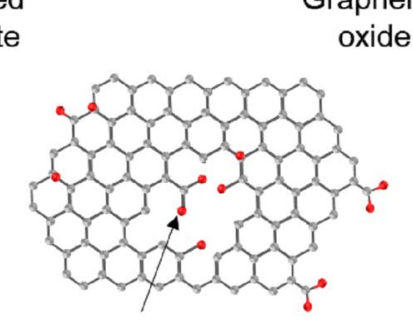

Graphene oxide defect

(c)
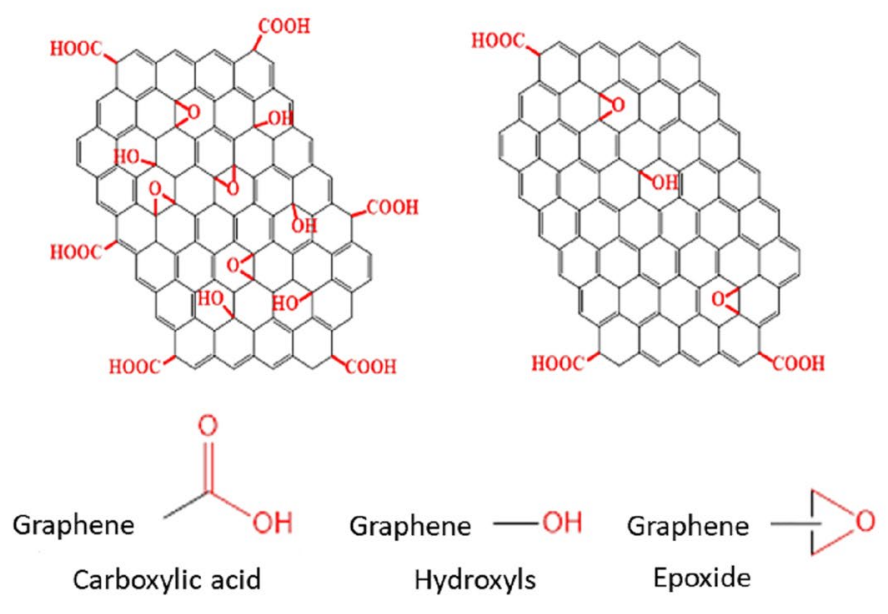

\section{Properties and methods of obtaining graphene}

According to Vieira and Vilar, the properties of graphene (thermal, electrical, and mechanical) make this product an excellent competitor for a variety of applications in the field of nanoscience and nanotechnology. The electrical conductivity (up to $2.104 \mathrm{~S} \mathrm{~cm}^{-1}$ ) and electronic mobility $\left(2.105 \mathrm{~cm}^{2} \mathrm{~V}^{-1} \mathrm{~s}^{-1}\right.$, which is more than 100 times greater than that of silicon) in the graphene monolayer result from a small effective mass. Since the electronic structure of a graphene monolayer overlaps two conical points in the Brillouin zone, charge carriers can be understood as "massless" or "no mass" electrons or Dirac fermions [85].

Graphene has a large specific theoretical area (2630 $\left.\mathrm{m}^{2} \mathrm{~g}^{-1}\right)$ [86], high intrinsic mobility $\left(200,000 \mathrm{~cm}^{2} \mathrm{~V}^{-1} \mathrm{~s}^{-1}\right)$ [87], Young's modulus (1.0 TPa) [88], thermal conductivity $\left(5000 \mathrm{~W} \mathrm{~m}^{-1} \mathrm{~K}^{-1}\right)$ [89]. Additionally, the optical transmittance $(97.7 \%)$ and good electrical conductivity deserve attention and support capacity and current density of $108 \mathrm{~A} \mathrm{~cm}^{-2}$, among several potential applications for applications such as transparent conductive electrodes. Bhuyan et al. reported that its potential could not be fully explored without economically developed surface techniques for synthesizing high-quality and high-area graphene [56].

The most common way of obtaining graphene is through graphite. However, it can also be obtained through recycling. Recent research shows that graphene allows the evaporation of water without allowing other liquids and gases to escape from the container, thus, making it fit for the desalination of sea water [90].

Different processes such as the adhesive tape method, exfoliation, mechanical delamination, silicon carbide synthesis, chemical vapor deposition (CVD), microwave treatment, oxidation, and chemical reduction methods have been explored by many researchers worldwide [91]. Graphene synthesis methods include top-down and bottom-up approaches. The first one involves breaking graphite stacked layers to yield graphene sheets, while in the bottom-up processes, one starts with structures smaller than graphene to reach the desired dimension $[92,93]$. Thus, for example, the exfoliation method is the primary representative of the top-down approach and CVD as a viable bottom-up method to obtain graphene. 
According to Reina et al. [94], CVD is a procedure considered inexpensive, which produces high-quality monolayer and low-layer graphene with a low number of defects being significantly useful for creating bioelectronic devices [94]. CVD is made of poly and monocrystalline metals, particularly copper or nickel surfaces. Additionally, when these substrates are unsuitable for end-use, transferring graphene methods to appropriate surfaces can be performed.

The wide variety of methods for synchronizing graphene in sizes, such as the process of mechanical exfoliation and cleavage, CVD, or chemical reduction of GO, can be grouped into two categories: bottom-up or down-up. The bottom-up approach synthesizes graphene starting with carbon atoms or molecules and accumulates in graphene deposited on a substrate. In contrast, in the top-down approach, a pattern generated on a large scale (graphite) is decreased to graphene [66].

There are four primary methods for oxidizing graphiteto-graphene oxide: Brodie (BRGO), Staudenmaier (STGO), Hofmann (HOGO), and Hummers (HUGO) methods [95-98]. These methods are performed in an acidic environment combined with potent oxidizing agents, such as potassium permanganate or potassium perchlorate. The technique used intensely influences the amount of specific functional groups $(\mathrm{OH}, \mathrm{COOH}$, ketones, and epoxides) and the degree of oxidation (C/O ratio) [12, 99]. Graphene sheets in different oxidation states can be obtained by modulating the other acid conditions in the exfoliation method. For example, GO with varying oxidation states was achieved at increasing graphite and $\mathrm{KMnO}_{4}(1: 2,1: 4,1: 6,1: 8,1: 10$, and 1:12) [100]. Theoretical calculations indicated that epoxy groups create a bridge between adjacent carbon atoms and enlargement in lattice constant and $\mathrm{C}-\mathrm{O}$ bond length and shortening of $\mathrm{C}-\mathrm{C}$ bond length during progressive oxidation.

\section{Structure of graphene oxide and theoretical simulation-models}

In 1939, Hofmann and Holstd built a structural GO model in which many epoxy groups were randomly distributed in the graphite layer [101], while Ruess and Vogt) updated this model by incorporating the hydroxyl entities and alternating the basal plane structure ( $\mathrm{sp}^{2}$ hybridized model) with $\mathrm{sp}^{3}$ hybridized carbon system [102]. Furthermore, Scholz and Boehm proposed a less ordered structure with double $\mathrm{C}-\mathrm{C}$ bonds and $\mathrm{C}=\mathrm{C}$ bonds periodically cleaved in the conjugated carbon layers, hydroxyl, and carbonyl groups in different environments, free of ether oxygen [103]. Additionally, Nakajima and Matsuo proposed a lattice structure similar to the graphite intercalating compound (GIC) in stage 2 . They suggested it because graphite oxide fluorination provides the same X-ray diffraction pattern as stage 2 graphite fluoride $\left(\mathrm{C}_{2} \mathrm{~F}\right)$ [15].
Lerf et al. characterize their GO by ${ }^{13} \mathrm{C}$ and ${ }^{1} \mathrm{H}$ nuclear magnetic resonance (NMR) and subsequently observed the $60 \mathrm{ppm}$ line best related to epoxide groups (1,2-ethers) other than 1,3-ethers, and the $130 \mathrm{ppm}$ line to aromatic entities and conjugated double bonds [104]. The carbon atoms attached to the $\mathrm{OH}$ groups slightly distorted their tetrahedral structure, resulting in partial wrinkles of the layers. They proposed an almost flat carbon grid structure with randomly distributed aromatic regions with benzene rings and six-membered aliphatic rings. Savazzi et al. reported that the Lerf and Klinowski model ( $\mathrm{L}-\mathrm{K}$ model) becomes one of the most acceptable for moderately oxidized samples. However, all the previous models could not explain the origin of GO planar acidity, a well-known chemical property for GO [105]. Szabó et al. proposed a slightly modified model based on Scholz-Boehm model. They were based on the results of the elementary analysis, transmission electron microscopy, X-ray diffraction, diffuse reflectance Fourier transform spectroscopy (FTIR) in the infrared, X-ray photoelectron spectroscopy, and electronic rotation resonance in addition to NMR. They then proposed a carboxylic acid-free model, comprising two distinct domains: related cyclohexyl species, interspersed with tertiary alcohols and 1,3-ethers, and a corrugated network of keto/quinoid species [106].

Table 1 shows the GO structure studied by a wide variety of methods. The results obtained by solid-state NMR [107, 108], X-ray photoelectron spectroscopy (XPS) and X-ray absorption edge spectroscopy (XANES) [109-111], infrared by FTIR [112], and Raman spectroscopy [113] suggest the best description of the GO leaf structure is given by the Lerf-Klinowski model [104] and updated by Gao et al. (2009). This model represents each GO particle by the basal plane of $\mathrm{sp}^{3}$ hybridized carbon atoms with chemically linked hydroxyl and epoxy functional groups located near each other on both sides of the plane [114]. Carboxyl groups terminate the edges of the basal plane with five- or six-membered lactol rings $(\mathrm{O}-\mathrm{C}-\mathrm{O})$, ketone, and tertiary alcohol ester [115]. Fuji and Enoki demonstrated that GO particles always contain some primary domains of grapheme [116]. The content and size of domains are related to the conditions of the GO exfoliation/oxidation process. The intensity of the $\mathrm{sp}^{3}$ hybridization of the $\mathrm{C}-\mathrm{O}$ bond that induces a structural distortion of the basal plane revealed by electronic energy loss spectroscopy (EELS) is related to the conditions for obtaining GO and the initial graphite structure [117].

One of the disadvantages of adding sodium nitrate in the Hummers method is that it can increase oxidation in the basal planes of graphite leaves, which has caused enormous stress resulting in rupture and peeling [118]. This way, it is advantageous to opt for a process based on the complete removal of $\mathrm{NaNO}_{3}$ [119] or to replace it with $\mathrm{K}_{2} \mathrm{~S}_{2} \mathrm{O}_{8}$ [120], $\mathrm{H}_{3} \mathrm{PO}_{4}$ [121], or $\mathrm{K}_{2} \mathrm{FeO}_{4}$ [19]. The elimination of nitrate is 
Table 1 Method of preparation of graphene oxide

\begin{tabular}{|c|c|c|c|c|c|}
\hline References & Carbon source & Oxidizing agents & $\begin{array}{l}\text { Time for } \\
\text { graphite } \\
\text { oxide }\end{array}$ & Temperature $\left({ }^{\circ} \mathrm{C}\right)$ & Factors \\
\hline Brodie [16] & Graphite & $\mathrm{KClO}_{3}, \mathrm{HNO}_{3}$ & 3/4 days & 60 & Oldest method \\
\hline Staudenmaier [17] & Graphite & $\mathrm{KClO}_{3}, \mathrm{HNO}_{3}, \mathrm{H}_{2} \mathrm{SO}_{4}$ & $96 \mathrm{~h}$ & Ambient temperature & Increased efficiency \\
\hline et al. [18] & Graphite-44micro & $\mathrm{KMno}_{4}, \mathrm{NaNO}_{3}, \mathrm{H}_{2} \mathrm{SO}_{4}$ & $<2 \mathrm{~h}$ & $<20-35-98$ & $\begin{array}{l}\text { No water and less than } 2 \mathrm{~h} \\
\text { of processing }\end{array}$ \\
\hline Fu et al. [303] & Graphite & $\mathrm{KMnO}_{4}, \mathrm{NaNO}_{3}, \mathrm{H}_{2} \mathrm{SO}_{4}$ & $<2 \mathrm{~h}$ & 35 & $\begin{array}{l}\mathrm{NaNO}_{3} \text { validated not } \\
\text { necessary }\end{array}$ \\
\hline Shen et al. [304] & $\begin{array}{l}\text { Colloidal graphite- } \\
\text { 10micro }\end{array}$ & Benzoyl peroxide & $10 \min$ & 110 & Fast and non-acidic \\
\hline Su et al. [305] & $\begin{array}{l}\text { Sonicated gra- } \\
\text { phene }<3000 \text { micro }\end{array}$ & $\mathrm{KMnO}_{4}, \mathrm{H}_{2} \mathrm{SO}_{4}$ & $4 \mathrm{~h}$ & Ambient temperature & Large graphene oxide \\
\hline Marcano et al. [121] & Graphite-150micro & $\mathrm{H}_{2} \mathrm{SO}_{4}, \mathrm{H}_{3} \mathrm{PO}_{4}, \mathrm{KMnO}_{4}$ & $12 \mathrm{~h}$ & 50 & $\begin{array}{l}\text { Less manganese impurity } \\
\text { and acid }\end{array}$ \\
\hline Sun et al. [142] & Expanded graphite & $\mathrm{KMnO}_{4}, \mathrm{H}_{2} \mathrm{SO}_{4}$ & $1.5 \mathrm{~h}$ & 90 & $\begin{array}{l}\text { High performance con- } \\
\text { fined to size and safe }\end{array}$ \\
\hline Eigler et al. [306] & Graphite-300micro & $\mathrm{KMnO}_{4}, \mathrm{NaNO}_{3}, \mathrm{H}_{2} \mathrm{SO}_{4}$ & $16 \mathrm{~h}$ & 10 & $\begin{array}{l}\text { High quality graphene } \\
\text { oxide }\end{array}$ \\
\hline Panwar et al. [307] & Graphite & $\begin{array}{l}\mathrm{H}_{2} \mathrm{SO}_{4}, \mathrm{H}_{3} \mathrm{PO}_{4}, \mathrm{KMnO}_{4}, \\
\mathrm{HNO}_{3}\end{array}$ & $3 \mathrm{~h}$ & 50 & High performance \\
\hline Peng et al. [308] & Graphite $>10$ micro & $\mathrm{K}_{2} \mathrm{FeO}_{4}, \mathrm{H}_{2} \mathrm{SO}_{4}$ & $1 \mathrm{~h}$ & Ambient temperature & $\begin{array}{l}\text { High performance and less } \\
\text { pollution }\end{array}$ \\
\hline $\begin{array}{l}\text { Rosillo-Lopez et al. } \\
\text { [309] }\end{array}$ & - & $\mathrm{HNO}_{3}$ & $20 \mathrm{~h}$ & Ambient temperature & Nano-sized GO \\
\hline Yu et al. [19] & Graphite—44micro & $\begin{array}{l}\mathrm{K}_{2} \mathrm{FeO}_{4}, \mathrm{H}_{2} \mathrm{SO}_{4} \\
\mathrm{KMnO}_{4}, \mathrm{H}_{3} \mathrm{BO}_{4}\end{array}$ & $5 \mathrm{~h}$ & $<5-35-95$ & $\begin{array}{l}\text { Less manganese impurity } \\
\text { and less acid }\end{array}$ \\
\hline Dimiev et al. [310] & Graphite-44micro & $\begin{array}{l}\left(\mathrm{NH}_{4}\right)_{2} \mathrm{~S}_{2} \mathrm{O}_{8}, \mathrm{H}_{2} \mathrm{SO}_{4}- \\
\text { Fumegante, } \mathrm{H}_{2} \mathrm{SO}_{4} \\
98 \%\end{array}$ & $3 / 4 \mathrm{~h}$ & Ambient temperature & High performance \\
\hline Pei et al. [311] & Graphite-44micro & $\mathrm{H}_{2} \mathrm{SO}_{4}$ & $<5 \min$ & Ambient temperature & $\begin{array}{l}\text { Slightly oxidized, } 25 \mathrm{~nm} \\
\text { thick, } 100 \% \text { conversion }\end{array}$ \\
\hline Ranjan et al. [312] & Graphite-44micro & $\mathrm{H}_{2} \mathrm{SO}_{4}, \mathrm{H}_{3} \mathrm{PO}_{4}, \mathrm{KMnO}_{4}$ & $>24 \mathrm{~h}$ & $<35 / 95$ & $\begin{array}{l}\text { Electrochemical support; } \\
\text { High efficiency and high } \\
\text { performance; Cool the } \\
\text { exothermic reaction to } \\
\text { keep safe }\end{array}$ \\
\hline Tian et al. [313] & Graphite -80 mesh & $\mathrm{H}_{2} \mathrm{SO}_{4}, \mathrm{NaNO}_{3}, \mathrm{KMnO}_{4}$ & $2 \mathrm{~h}$ & Ambient temperature & $\begin{array}{l}\text { Good application in super- } \\
\text { capacitors }\end{array}$ \\
\hline Hou et al. [314] & Graphite & $\mathrm{H}_{2} \mathrm{SO}_{4}, \mathrm{NaNO}_{3}, \mathrm{KMnO}_{4}$ & $4 \mathrm{~h} / 30 \mathrm{~min}$ & $35 / 95$ & $\begin{array}{l}\text { High performance and bet- } \\
\text { ter controllable structure }\end{array}$ \\
\hline Silva et al. [315] & $\begin{array}{l}\text { Graphite powder (CBG } \\
\text { Mining) }\end{array}$ & $\mathrm{H}_{2} \mathrm{SO}_{4}, \mathrm{KMnO}_{4}$ & $10 \mathrm{~min}$ & 70 & $\begin{array}{l}\text { Microwave digestion; GO } \\
\text { nanosheets about } 0.9 \mathrm{~nm} \\
\text { thick }\end{array}$ \\
\hline
\end{tabular}

Adapted and updated from Sun [316]

more ecological, as it completely stops the production of toxic gases such as $\mathrm{NO}_{2}$ and $\mathrm{N}_{2} \mathrm{O}_{4}$ [121].

Additionally, it avoids the absorption of nitrogen contamination in the GO structure. Recently, it was demonstrated that the exclusion of $\mathrm{NaNO}_{3}$ does not affect the yield of the general reaction, and GO is obtained with almost the same properties as conventional methods [119]. Nitrate-free approaches are generally lighter than the original Hummers method. The careful control of water quantity and reaction temperature leads GO with hydroxyl and epoxy rich GO domains or more carbonyl rich GO domains (Fig. 4), respectively [122].

\section{${ }^{13} \mathrm{C}$ nuclear magnetic resonance in the solid-state}

$\mathrm{GO}$ is one or a few thick atomic layers of carbon atoms attached to different groups, such as epoxy, hydroxyl, carbonyl, and carboxyl. The exact structure of GO is still a 
Fig. 4 The synthesis of GOs with controlled species of oxygenated groups. Adapted from Jin et al. 2011 [173]

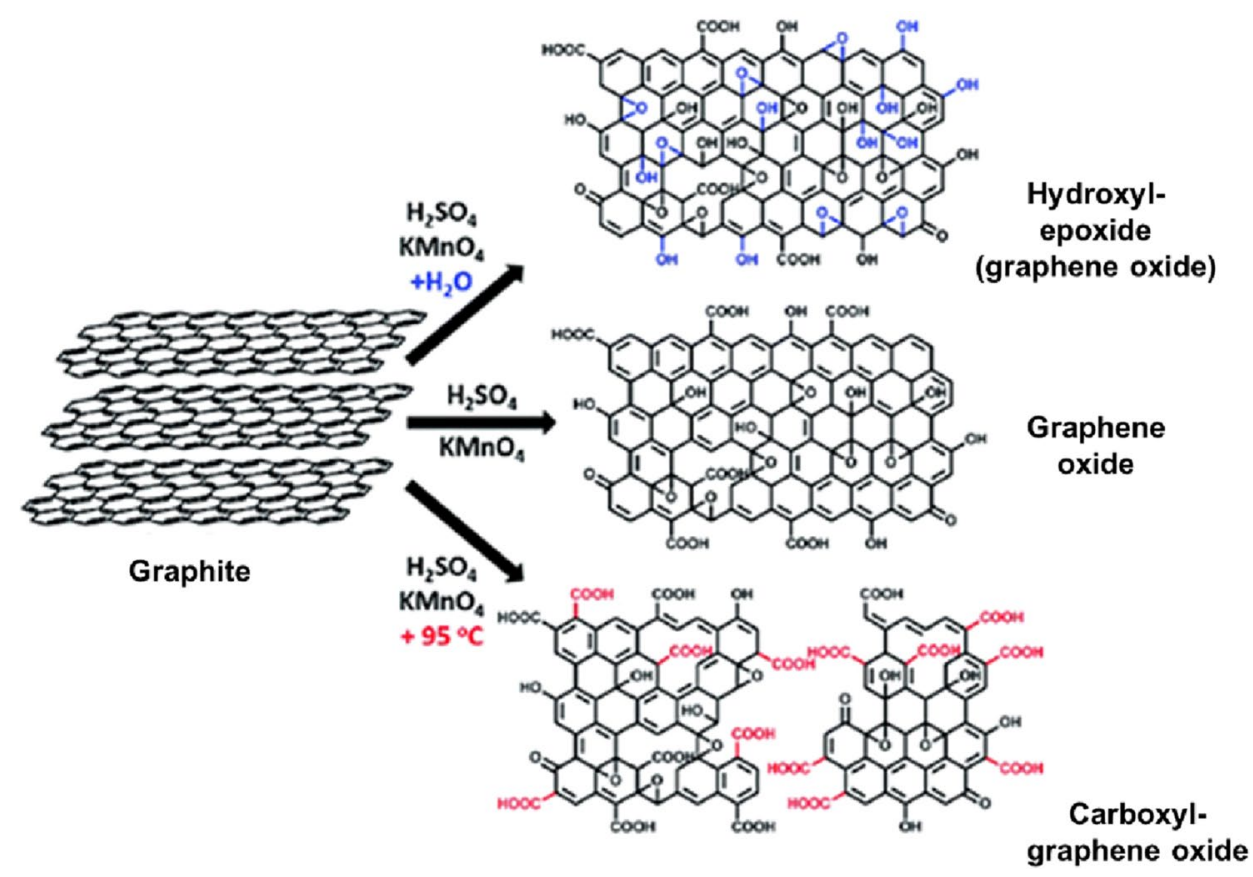

cause for debate. The nature, quantity, and distribution of the different groups in GO depend on the synthesis method, while the reduction depends on the process and the nature of the reducing agent [106]. Nuclear magnetic resonance in the solid-state (NMR) is a good technique for analyzing the structure of the materials. The ${ }^{13} \mathrm{C}$ and ${ }^{1} \mathrm{H}$ NMR spectra of GO exhibit three prominent peaks at 60, 70, and $130 \mathrm{ppm}$ [117]. In some studies, four peaks were found at 57.6, 69.2, 92.9, and $166.3 \mathrm{ppm}$. In high resolution, the ${ }^{13} \mathrm{C}$ NMR spectra of GO also shown three smaller peaks at 101,167 , and 191 ppm, respectively [118].

GO leaves are gigantic molecules that fall into the category of colloids. GO dispersions in water are too diluted to be analyzed by the Nuclear magnetic resonance in a liquid phase. In the literature, the most powerful and precise technique for characterizing $\mathrm{GO}$ is solid-state ${ }^{13} \mathrm{C} \mathrm{NMR}$. Due to the low natural abundance of ${ }^{13} \mathrm{C}(1.1 \%)$, the signal-tonoise ratio is low when measuring regular samples. The long acquisition time is usually a necessity for good quality data. Therefore, Cai et al. prepared a C-enriched GO sample and clarified its chemical structure to a new level [107].

Interestingly, although the isotopic labeling of GO has dramatically improved the resolution of the solid-state nuclear magnetic resonance (SSNMR) analysis, there are still some unassigned peaks in this work. Subsequently, other reports were published with more detailed attributions of those peaks, such as a new identification of the 101-ppm peak, which has long been ignored by previous researchers [121].

Figure 5 shows a typical comparison of a cross-polarization spectrum from ${ }^{1} \mathrm{H}$ to ${ }^{13} \mathrm{C}$ and a direct pulse of ${ }^{13} \mathrm{C}$

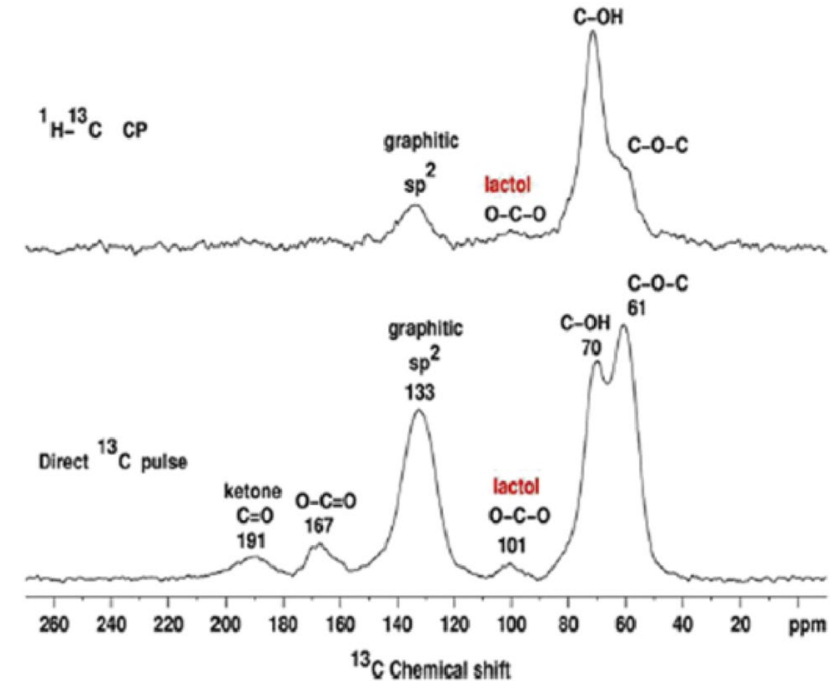

Fig. 5 A 1 H-13 C cross-polarization (CP) GO spectrum obtained with $7.6 \mathrm{kHz}$ MAS and a contact time of $1 \mathrm{~ms}(67,000$ scans, at the top) and a $13 \mathrm{C}$ direct pulse spectrum obtained with $12 \mathrm{kHz}$ MAS and a $90^{\circ} 13 \mathrm{C}$ pulse $(10,000$ scans). The peak at $101 \mathrm{ppm}$ is caused by five-carbon and six-membered ring lactols. Adapted from Georgakilas et al. 2012 [174]

spectrum obtained from unmarked GO, with quantitative data on the relative ratio of all these functionalities to 115 (hydroxyl and epoxy): 3 (lactol O-C-O): 63 (graphitic carbon $\mathrm{sp}^{2}$ ): 10 (lactol + ester + acid carbonyl): 9 (carbonyl ketone) [123].

More advanced SSNMR techniques were later used for GO characterization, including $2 \mathrm{D}-{ }^{13} \mathrm{C}$ double quantum/ 
single-quantum (2Q/SQ) correlation, SSNMR, ${ }^{13} \mathrm{C}$ displacement chemical anisotropy change (CSA) 2D SSNMR/correlation of isotropic change SSNMR, and triple quantum 2D/correlation of single quantum (3Q/SQ) SSNMR [108]. The 2Q/SQ spectrum eliminates diagonal signals in the $2 \mathrm{D}$ spectrum, offering a clearer correlation signal between carbon ${ }^{13} \mathrm{C}-\mathrm{OH}$ and carbon ${ }^{13} \mathrm{C}-\mathrm{O}-{ }^{13} \mathrm{C}$ (Fig. 6).

Interestingly, the theoretical simulation was used simultaneously to fit these data with simplified GO structure models and satisfactory fit obtained by calculations, with the structure model A (with only 1, 2-ether). All of these analyzes significantly helped to identify the chemical structure of GO, leading to greater clarity in its chemical compositions. However, it is also important to note that all these SSNMR characterizations were made on GO samples manufactured using the modified Hummers method. The GO products from other methods differ in the relative proportion of these features [121].
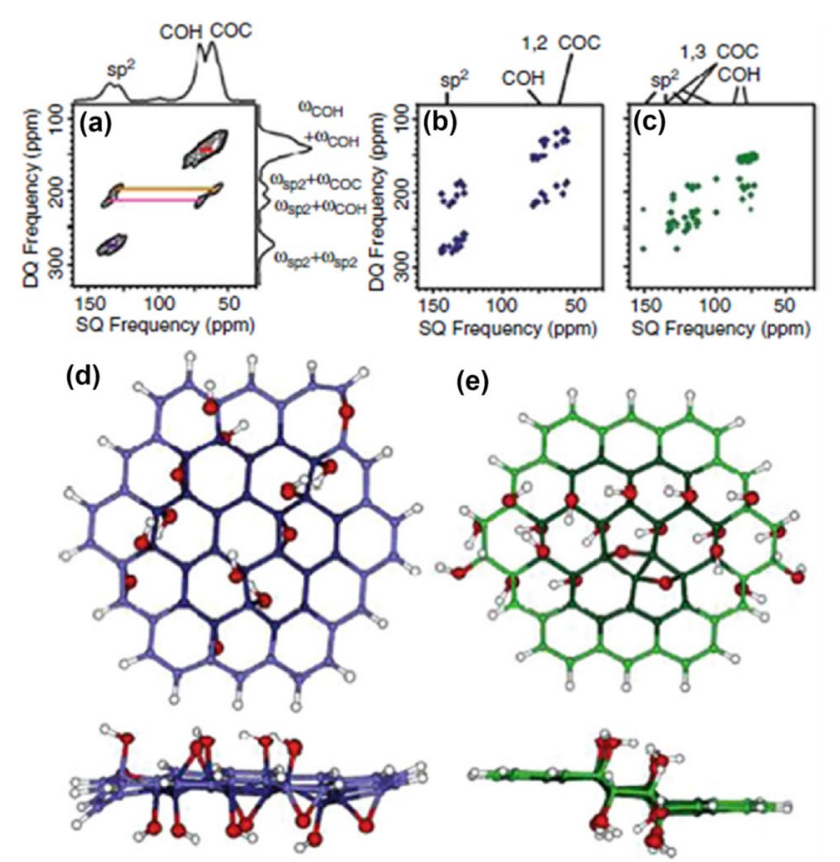

Fig. 6 a Experimental spectrum SSNMR correlation $2 D^{13} C D Q / S Q$ uniformly labeled with $13 \mathrm{C}$ VAI using coherence transfer from ${ }^{13}$ to ${ }^{13} \mathrm{C} \mathrm{J}$. Fast recycling with short recycling delays of $0.3 \mathrm{~s}$ and low power. Decoupling $(7 \mathrm{kHz})$. The signal designations in a are those made by Hummers and Offeman (1958) and confirming the carrier frequency was established at $211.17 \mathrm{ppm} ; \mathbf{b}$, c DQ/SQ correlation predicted spectra based on isotropic chemical shifts calculated for b model A and c model B; (d, e). Structural models for $\mathbf{d}$ model A and $\mathbf{e}$ model $\mathrm{B}$ from the upper and lower side views. Carbons in $\mathbf{d}, \mathbf{e}$ are color-coded to match the spectra in $(\mathbf{b}, \mathbf{c})$. Red and white spheres denote $\mathrm{O}$ and $\mathrm{H}$, respectively. ${ }^{13} \mathrm{C}$ at the edge of the models (light blue or green) was not included in $(\mathbf{b}, \mathbf{c})$. The calculated spectrum b based on model A reproduced the experimental spectrum a well. Adapted from Chen et al. 2013 [119]
Functionalization of graphene-based nanomaterials

The chemical modification of graphene surface, by the insertion of molecules, polymers, copolymers, macromolecules, among other chemical compounds, improves its properties and behavior, promoting characteristics suitable for medical applications [124, 125].

Figure 7 shows the main altered graphene characteristics by its functionalization, comprises its toxicity reduction due to the coupled agents biomimicry and the ability of cellular capture profile. Changes promoted by functionalization of nanosystems uptake have significant interest for the biomedi$\mathrm{cal}$ area. These changes can make nanocomplexes more specific to drugs, genes, or imaging agent applications [126], preventing uptake by phagocytic cells of the reticuloendothelial system [127], and inhibit the binding of opsonin proteins, which lead to recognition by phagocytic cells [128]. Consequently, there is an increase in the circulation time of the nanosystems, and their active targeting results in lesser side effects, reduced doses, greater adherence, and, finally, improvement in the patient's quality of life [129].

Graphene-based nanomaterials require strategies for the functionalization of their surface that allows reaching and exploiting their full potential. Hence, approaches and procedures are developed to expand and improve its characteristics, enabling its applications in materials engineering and nanotechnology.

\section{Methodologies to functionalize graphene and its derivative}

Covalent method Numerous functional groups can be anchored to GO and rGO through typical organic reactions, such as amidation, esterification, and silanization, covering a wide range of molecules, including all types of aliphatic and aromatic amines, amino acids, biomolecules, silanes, polymers, and some nanoparticles [130]. For the characterization of the products obtained by the functionalization of

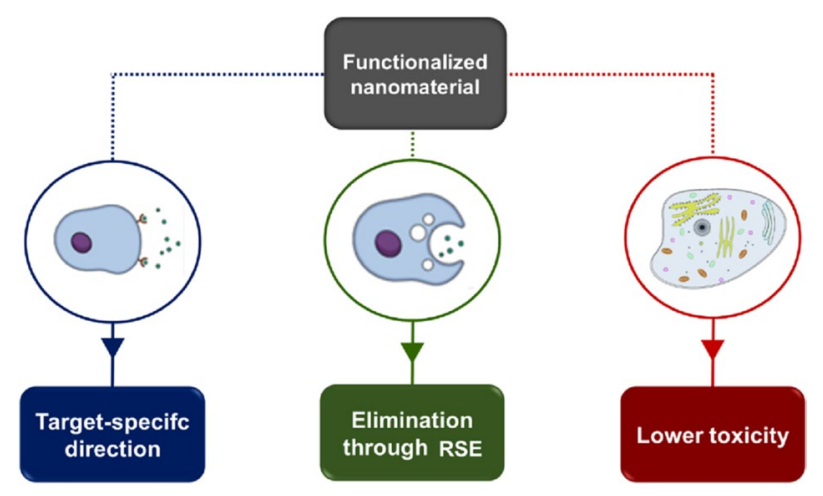

Fig. 7 Main effects of nanomaterials functionalization. Source: authors 
graphene through the covalent method, the electron microscopy techniques, Raman spectroscopy, and infrared spectroscopy are the primary tools applied.

Amidation According to the conventional method, the chemical modification through amidation consists of a two-step synthesis [131]. As shown in Fig. 8, first, the carboxylic acid groups on the nanomaterial surface are activated using coupling agents, such as carbodiimide derivates (EDC or DDC). The nucleophilic addition is performed, in which the nucleophile, represented by the amino group $\left(\mathrm{R}^{2}-\mathrm{NH}_{2}\right)$, attacks the carbonyl of activated-carboxylic acid groups of $\mathrm{GO}$ molecules $\left(\mathrm{R}^{1}-\mathrm{COOH}\right)$.

Graphene amidation allows the synthesis of a material with a wide variety of applications. One of these applications is biosensors production, whose purpose is to detect a specific analyte to quantify it or monitor or diagnose a particular health condition. In this regard, several studies [132-134] exemplified this strategy. Li et al. developed a thrombin biosensor composed of graphene, linked to a double-stranded DNA, and gold nanoparticles linked to the thrombin-binding aptamer [135]. DNA has the function of facilitating the dispersion of GO in the physiological medium and is linked covalently through the amidation reaction. On the other hand, gold nanoparticles provide the appropriate microenvironment for immobilizing DNA and facilitate the transfer of electrons between it and the electrodes. Begun et al. performed the immobilization of chitosan in GO surface through the amidation reaction between chitosan amine and nanomaterial carboxylic groups, allowing the production of a matrix for simultaneous detection of dopamine and uric acid [132].

Ye et al. reported the functionalization of GQDs with bovine serum albumin to identify and quantify tryptophan enantiomers. Yang et al. developed rGO functionalized with polyaniline, a nitrogenous polymer, for the detection of procalcitonin for early diagnosis of sepsis $[136,137]$. As a result, gold nanoparticles were anchored to graphene already functionalized to promote the fixation of the components responsible for detecting and quantifying the biomarker.

In addition, to studies related to biosensors, there is also interest in using these nanocomplexes in the targeted transport of drugs, imaging agents, and genes. Several studies have investigated this application. As a new gene nanocarrier, Imani et al. explored the viability of GO functionalized with octaarginine conjugated amidation [138].

Abdollahi et al., in a study, coated nanocomplexes of GO coupled to iron oxide with poly (ethylene glycol) bis (amine)
(PEGA), covalently linked to graphene from amide bonds, in addition to being related to the drug. This nanoplatform was used as a methotrexate carrier to increase cytotoxicity to tumor cells, increase stability, blood circulation time, and biocompatibility [139].

In their study, Liu et al. developed a docetaxel co-targeting nanosystem, an antineoplastic, with genes (MMP-9 shRNA) associated with apoptosis and tumor cell metastasis. This nanosystem consists of GO functionalized with highly branched poly(amidoamine) (HPPA) by the covalent method. The amidation reaction occurs with the formation of an amide bond between the graphene and HPPA [140]. Finally, Yang et al. developed a functionalized graphene nanoplatform capable of carrying the drug pirfenidone to improve the treatment of subarachnoid hemorrhage. This nanoplatform was produced from amidation reactions between the carboxylic groups of GO and amino groups of the amino methoxy poly (ethylene glycol) $\left(\mathrm{mPEG}-\mathrm{NH}_{2}\right)$ [141].

Another pertinent use of this nanomaterial is to develop material to assist in the remediation of water through the adsorption of contaminants. Sun et al. developed rGO hydrogel functionalized with triethylenetetramine (TETA) capable of removing pollutants from Copper (II) and ciprofloxacin (an antimicrobial drug) from aqueous solutions [142]. In addition, Wang et al. developed a new magnetic nanodevice synthesized with GO covalently functionalized with poly(ethyleneimine) (PEI) [143]. This nanoplatform was established to remove hexavalent chromium from water and soil.

Esterification The esterification reaction is another route used to change the chemistry of graphene. Its application was observed in several areas, such as the development of a biosensitivity [144], used for quantification of di (2-ethylhexyl) phthalate (DEHP). This plasticizing agent can migrate from the packaging to the product and cause various health problems. This biosensor consists of GO functionalized with dendrimer molecules of polyamine terminated with ferrocenecarboxylic acid (FcAED). This functionalization occurred through the esterification reaction between the carboxylic terminal groups of the FcAED molecule and the hydroxyl groups on the GO surface.

Li explored a GO compound covalently coupled to l-borneol, a monoterpene in essential oils from plants such as Dryobalanops aromatic and Mentha spicata, with described antimicrobial and antifungal activity [145]. First, GO was
Fig. 8 General mechanism of the amination reaction; $R$ Compound to be inserted. Source: authors

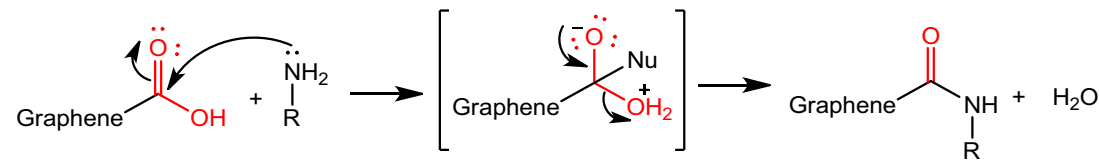


modified with thiomalic acid, which promotes the opening of the epoxide ring and binds to free $\mathrm{sp}^{3}$ carbon to increase the available carboxylic groups in the nanomaterial, potentiating the esterification reaction with borneol. This alcohol will react both with the carbonyls of thiomalic acid anchored on the nanomaterial surface and with the carbonyls of the carboxylic groups of GO. In this way, a nanoplatform capable of inhibiting, in vitro tests, the adhesion and growth of the fungus Mucor racemosus was developed. Meng et al. performed the functionalization of GO by esterifying it with $\beta$-cyclodextrin $(\beta-C D)$, thus producing a nanosystem with great potential for targeting drugs. $\beta$-CD is a cyclic oligosaccharide composed of six units of D-glucose that has a hydrophobic interior and hydrophilic exterior [146].

Sarvari et al. explored the use of graphene nanomaterials in the production of scaffolding used in tissue engineering has been investigated and performed the esterification of rGO with 3-thiophene acetic acid (TAA), to be used later as an initiator of the subsequent polymerization reactions with poly (3-dodecyl thiophene) and poly (3-thiophene ethanol) [147]. Additionally, Wu et al. developed a porous scaffold for use in the engineering of bone or cartilage tissues, formed by starch covalently linked with GO through the ester link between the hydroxyls of the glucose units and the carbonyls of the carboxylic acids present in the nanomaterial [148].

Raheem et al. produced a new electrode based on graphene esterified with glycerol, which detects electrocardiogram signals with high conductivity and less noise, comparatively more efficient than the commercially available electrode. This fact occurs because glycerol is conjugated to nanomaterial through three groups of carboxylic acids present in different sheets of GO connecting them, thus increasing the conductivity of the device [149].

Silanization Organosilane (Fig. 9) are used to form covalent bonds between silicon and GO. Organosilane is a monomer in which silicon is attached to hydrolysable groups and nonhydrolysable groups. The general formula of an organosilane is $\mathrm{RSiY}_{3}$, where $\mathrm{Y}$ is a hydrolysable group, which can be halogen, alkoxy (-CO-), acyloxy (-COO-) or amine ($\mathrm{NH}_{2}$ ), and the group $\mathrm{R}$ is a non-hydrolysable group, which provides the desired characteristics to the final compound [150].

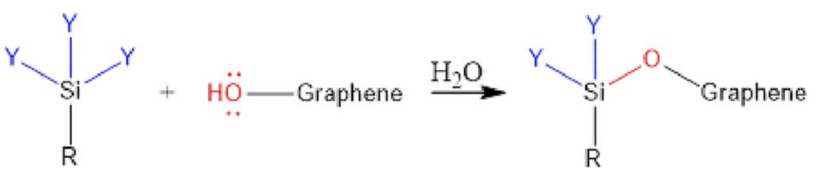

Fig. 9 Reaction of silanization, whose mechanism is not elucidated. Source: authors
Wen et al. developed a complex nanoplatform, in which graphene-based nanocarrier is functionalized with 3-(2-aminoethylamino) propyltrimethoxysilane (AEAPTMS) are incorporated into spheres of mesoporous silica. The objective of using functionalized graphene nanomaterial from silanization is to produce multifunctional nanoplatforms capable of providing high luminescence without unwanted effects, such as blinking or photobleaching, and enabling the transport of substances, such as drugs or other diagnostic agents [151].

In several studies by Hassan et al. (2020) and Yu et al. (2017), graphene nanoplatforms functionalized with silane groups were used to remove metals from aqueous solutions. Hassan et al. developed a new hybrid system composed of a magnetic material (magnetic sporopollenin) coupled to GO through the 3-chloropropyltrimethoxysilane silane group (CTPS), covalently linked to graphene. This hybrid material has improved efficiency for removing Lead (II) from the aqueous medium [152]. Song et al. [153] used Fe3O4modified ultrasmall graphene oxide to eliminate ppm-level pollutants (methylene blue) [154]. Yu et al. prepared a new nanosystem through the silanization reaction between organosilane, $\gamma$-(2,3-epoxypropoxy) propyltrimethoxysilane coupled to $\beta$-cyclodextrin ( $\beta$-CD /GPTMS) and GO for removing copper (II) ion in water [155]. Song et al. [156] have demonstrated the ability of PEGylated-graphene oxide (PEG-GO) to remove water-insoluble pesticides. In this direction, by use of carboxymethyl chitosan-modified carbon nanoparticles (CMC@CNP) have been used to remove emamectin benzoate (widely used insecticide) [157]. Mahmoud et al. [158] have used multifunctionalized GO to remove dyes (crystal violet and black 5), with success. In this direction Malinga and Jarvis [159] used rGO for removal of $\mathrm{Cr}(\mathrm{VI})$. The results showed that rGO could be used, especially in association with Cobalt (complex Co-rGO) as a recycle nanosystem for $\mathrm{Cr}(\mathrm{VI})$ removal. Finally, Menezes et al. have demonstrated the use of GQDs to remove radioactive waste materials, especially uranium $\left({ }^{238} \mathrm{U}\right)$, from complex liquids [160].

Noncovalent method This method offers a means to enhance and adjust the properties of graphene without changing its chemical structure and conductivity since it is based on the adsorption of surfactants, polymers, and biomolecules. Stabilization using surfactants occurs by the formation of micelles or hemi-micelles on the surface of the nanosheet. Thus, interactions between surfactant molecules and graphene can be exploited to achieve desirable colloidal stability [161]. On the other hand, hydrophilic and amphiphilic polymers are efficiently absorbed over the sheets to provide a steric barrier and prevent restacking [161]. In addition, biomolecules such as proteins [162], peptides [163], and vitamins [164] are attractive from the standpoint of their 
safety and non-immunogenicity, enabling the biomedical applications of graphene.

As explained in the previous section, the covalent method occurs by reaction between the oxygenated groups on the GO surface and the functional groups of specific molecules. Meanwhile, the noncovalent method is based on the electrostatic interaction and van der Waals forces. This process does not change the chemical structure, and it is easy to perform [165]. Baek et al. report a simple and generalized synthesis method for preparing nanoparticle/exfoliated graphene (EG) composites by tailored electrostatic interactions [166]. In this work, the authors introduced $\mathrm{Cl}^{-}$and $\mathrm{MoS}_{4}{ }^{2-}$ anions on the surface of metals, semiconductors, and magnetic nanoparticles by a two-phase ligand exchange reaction. Negatively charged nanoparticles were selectively assembled at the positively charged edges of exfoliated graphene treated with polyethyleneimine (PEI) without aggregation.

Intermolecular forces are interactions between molecules and are decisive for defining the macroscopic properties of the substance, such as physical state, viscosity, melting point, and boiling point. The nature of the interaction, polarizability, and contact area are the parameters that define its intensity. The main intermolecular interactions observed in the noncovalent modification of graphene are (1) $\pi-\pi$ interactions and (2) hydrogen bonding.

The $\pi-\pi$ interactions are a type of dispersion force applied to aromatic compounds. Dispersion forces occur between non-polar compounds. At first, it could be considered that there are no intermolecular interactions between non-polar molecules since these interactions are electrostatic, and nonpolar molecules do not have real or partial dipoles since the charge distribution is homogeneous. However, a particular concentration of electrons occurs in one specific region of the molecule at a particular moment. This way, one part will have a partial negative charge and another, a partial positive charge, momentarily generating an instantaneous dipole, spreading to neighboring molecules. This instantaneous dipole originates due to the property of polarizability, which can have the electronic cloud distorted, which depends on a short distance between the molecules [167].

The hydrogen bond is a particular case of dipole-dipole interaction, which is represented by $\mathrm{X}-\mathrm{H}-\mathrm{Y}$, where the hydrogen atom $(\mathrm{H})$ is between two electronegative species and has a pair of free electrons (X and $\mathrm{Y}$ ), which can be oxygen or nitrogen [168]. The electrostatic nature of these interactions has energy less than $20 \mathrm{~kJ} \mathrm{~mol}^{-1}$, which is below the energy present in chemical bonds, which are in the order of $250 \mathrm{~kJ} \mathrm{~mol}^{-1}$. When combined, they can be as strong as some covalent bonds. This method is the strategy of many researchers to produce new materials based on graphene using the noncovalent approach [55]. In this context, Niu et al. used this method to functionalize the pristine graphene through $\pi-\pi$ interactions with 3,4,9,10-tetracarboxylic acid
(PTCA). This process allows the introduction of carboxylic groups to the nanomaterial without causing deformations in its structure and promoting the amidation reaction between the $\mathrm{CH}$ amino group and the perylene carboxylic acid. As a result, they developed a biosensor to detect and quantify tryptophan enantiomers [133].

$\mathrm{Oz}$ et al. reported the noncovalent association of a maleimide-catechol compound (DOPA-MAL) with rGO from $\pi-\pi$ interactions and hydrogen bonds. This nanosystem has been associated with doxorubicin (DOX) to evaluate the treatment of cervical cancer (HeLa) and breast cancer (MDA-MB-231) [169].

Díez-Pascual and Díez-Vicente developed scaffolding for the engineering of synthesized fabrics based on the incorporation of poly (propylene fumarate) (PFF) and poly (ethylene glycol) (PEG) in GO sheets, through van der Waals and numerous hydrogen bonds between nanomaterial and polymers, and between the polymers themselves. Tensile tests showed strong adhesion of polymers to GO and good stiffness and resistance, including biological media, which confirms the ability of this nanoplatform to provide adequate support for the formation of bone tissue [170].

Pristine graphene Pristine graphene has a rich aromatic system and a planar structure, in addition to a strongly hydrophobic character. Moreover, rGO has less aromatic character, limited solubility in an aqueous medium, and still tends to aggregate through $\pi-\pi$ interactions. On the other hand, GO has deformations in the planar structure due to the high density of $\mathrm{sp}^{3}$ carbons, resulting from the insertion of functional groups containing oxygen, making it more soluble in water [130]. The graphene family's large surface and the predominantly planar area provide a platform that makes it possible to anchor several chemical species.

There are two main functionalization methods shown in Fig. 10: (1) covalent method, which involves the oxygenated groups of graphene, and (2) noncovalent method, which occurs through intermolecular interactions. The main advantage of using techniques related to hydrophobic or electrostatic interactions is that it does not promote changes in the structure of graphene. However, there are some

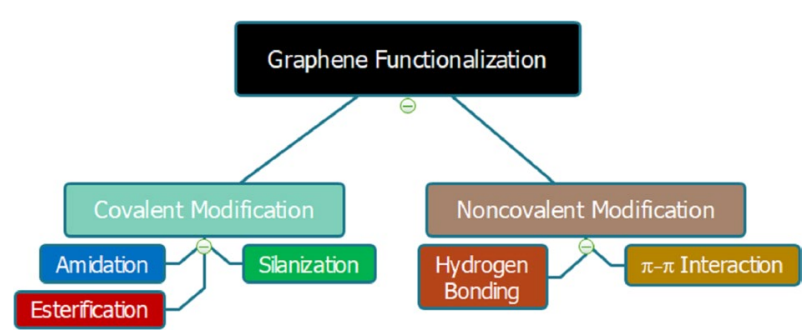

Fig. 10 Methods and mechanisms for the functionalization of graphene nanomaterials. Source: authors 
disadvantages to the noncovalent method that are related to its binding strength. Noncovalent interactions are not as strong as covalent bonds, making the nanoplatform less robust and less stable in vitro and in vivo when interacting with biological systems. On the other hand, covalent functionalization, much explored with the use of GO and rGO, disturbs the $\pi$ system, which means that essential properties such as electrical conductivity and mechanical resistance will be affected [171].

The covalent functionalization of the carbon skeleton can be carried out through an electrophilic attack of the $\mathrm{C}=\mathrm{C}$ bond in the aromatic ring of pristine graphene [172]. Zhong et al. prepared solution-phase graphene dispersed in a $2 \%$ sodium cholate aqueous solution and stirred dispersion 4-propargyloxydiazobenzenetetrafluoroborate as a functionalizing agent at $45{ }^{\circ} \mathrm{C}$ for about $8 \mathrm{~h}$, obtaining 4-propargyloxyphenyl graphene [173]. On the other hand, graphene's organic covalent functionalization reactions also include covalent bonds between free radicals or dienophiles and $\mathrm{C}=\mathrm{C}$ bonds of pristine graphene [174]. When a diazonium salt is heated, a highly reactive free radical is produced, attacking the $\mathrm{sp}^{2}$ carbon atoms of graphene, forming a covalent bond. Tour and co-workers have used this methodology to design graphene with nitrophenyls $[136,175]$. Niyogi and co-workers also showed that the covalent attachment of nitrophenyls to graphene sheets forms a controllable bandgap, making the functionalized graphene potentially useful as semiconducting nanomaterials [137].

Tables 2 and 3 illustrate the general mechanism of the main reactions involved in the functionalization of graphene by the covalent method, intermolecular interactions related to the noncovalent process, and changes observed in data analysis. Some research that developed functionalized graphene nanoplatforms using these methods will be further discussed, evidencing this topic's importance and deepening the knowledge about it.
Table 2 Main functions through the covalent pathway of grapheme oxide and its applications

\begin{tabular}{|c|c|c|c|}
\hline \multicolumn{4}{|c|}{ Covalent functioning method } \\
\hline Mechanism & Reagents & Application & References \\
\hline \multirow[t]{12}{*}{ Amination } & DNA & Biosensor & Li et al. [135] \\
\hline & Chitosan & & Begum et al. [132] \\
\hline & BSA & & Ye et al. [134] \\
\hline & PANI & & Yang et al. [317] \\
\hline & Octa-arginine & Carrier & Imani et al. [138] \\
\hline & PEGA & & Abdollahi et al. [139] \\
\hline & HPAA & & Liu et al. [140] \\
\hline & $\mathrm{mPEG}-\mathrm{NH}_{2}$ & & Yang et al. [141] \\
\hline & TETA & Water of remediation & Sun et al. [318] \\
\hline & PEI & & Wang et al. [143] \\
\hline & $\mathrm{DABu}$ and $\mathrm{DABe}$ & Catalysis & Ahmed and Kim [319] \\
\hline & TPAPAM & Electronic device & Fan et al. [320] \\
\hline \multirow[t]{11}{*}{ Esterification } & FcAED & Biosensor & Xiao et al. [144] \\
\hline & Borneol & Carrier & Li et al. [145] \\
\hline & $\beta$-Cyclodextrin & & Meng et al. [146] \\
\hline & TAA & Tissue engineering & Sarvari et al. [147] \\
\hline & Starch & & Wu et al. [148] \\
\hline & Polyglycerol & Water of remediation & Yu et al. [321] \\
\hline & TBPP & & Yao et al. [322] \\
\hline & $\begin{array}{l}\mathrm{PPy}-\mathrm{COOH} \text { and } \mathrm{PPy}- \\
\mathrm{COOCl}\end{array}$ & Catalysis & $\begin{array}{l}\text { Luceño-Sánchez and } \\
\text { Díez-Pascual [323] }\end{array}$ \\
\hline & Benzyl alcohol & Electronic device & Tachi et al. [324] \\
\hline & Glycerol & & Raheem et al. [149] \\
\hline & PVA & & Li et al. [325] \\
\hline \multirow[t]{4}{*}{ Silanization } & AEAPTMS & Theranostics & Wen et al. [151] \\
\hline & CPTS & Water of remediation & Hassan et al. [152] \\
\hline & $\beta$-CD/GPTMS & & Yu et al. [155] \\
\hline & GPTMS-MTES & Electronic device & Lee et al. [326] \\
\hline
\end{tabular}


Table 3 Main noncovalent features of the graphene family and its applications

\begin{tabular}{|c|c|c|c|c|}
\hline \multicolumn{5}{|l|}{ Noncovalent method of functionalization } \\
\hline Interactions & Reagents & Graphene derivatives & Application & References \\
\hline$\pi-\pi$ interactions & Chitosan & rGO-PTCA-CD & Biosensor & Niu et al. [133] \\
\hline \multirow[t]{3}{*}{$\pi-\pi$ interactions and hydrogen bonds } & DOPA-MAL & rGO/dopa-MAL-c & Carrier & Oz et al. [169] \\
\hline & PPF & PPF/PEG-GO & Tissue engineering & $\begin{array}{l}\text { Díez-Pascual and } \\
\text { Díez-Vicente } \\
\text { [170] }\end{array}$ \\
\hline & AAPDI & CdS/A-GO & Catalysis & Zhu et al. [327] \\
\hline$\pi-\pi$ interactions & PDI & $\mathrm{PDI} / \mathrm{G}$ & Electronic device & Nuin et al. [328] \\
\hline \multirow[t]{2}{*}{$\pi-\pi$ interactions and hydrogen bonds } & APy & PK/GNP/APy & Electronic device & Cho et al. [329] \\
\hline & $\mathrm{AP}-\mathrm{DSS}$ & rGO-AP-DSS & & Ni et al. [330] \\
\hline
\end{tabular}

\section{Biomedical applications of graphene and its derivatives}

\section{Graphene oxide (GO)}

GO has been increasingly used in biomedical research since graphene lacks surface regularity, sharp edges, cavities, and different distances between carbon atoms. These factors can lead to the rupture of the cell membrane's lipid bilayer in the human body, interrupting the transport of electrons in the mitochondrial respiratory chain. Consequently, there is cellular activation of the apoptosis pathway. Additionally, the hydrophobic nature of the nanomaterial results in a lack of solubility in most solvents, significantly limiting its use $[68,176,177]$. Figure 11 shows the possible interaction between graphene family nanomaterials and cell membrane as cytotoxicity effect.

The main difference between GO and its derivatives to graphene is that the first group has a fine atomic sheet structure containing optimized oxygenated groups so, GO can be used in adsorption. There is also a high surface area leading to various applications, including drug delivery and bioimaging [68, 168, 178-183] and an improvement in its aqueous dispersibility and reduction in the aggregation of nanoparticles, decrease in toxicity, and increase in its selectivity $[168,179,180,183]$. These properties led to GO being investigated for targeted cell delivery of anticancer drugs [178-184] and to function as an antibacterial agent [185, $186]$.

The targeted drug administer Hummers aims to achieve the therapeutic target selectively and at a controlled drug release rate. This system improves therapeutic efficacy and minimizes the associated side effects [182-184, 187, 188]. This system has been extensively studied to reduce dose-limiting cytotoxicity and decrease drug resistance [182, 184].

In general, the system consists of a cell recognition portion of interest and a cytotoxic portion connected directly or through a suitable ligand to form a conjugate [178, 184] occurring in two ways: by modifying the surface of the nanoparticle with targeting ligands or in response to the generated microenvironment, such as low $\mathrm{pH}$ and overexpression of a given biomarker [178, 182, 189-191].

Rao et al. developed a new anticancer drug carrier system with controlled and targeted delivery capabilities [183]. Carboxymethylcellulose and GO were covalently conjugated by generating $\mathrm{GO} / \mathrm{CMC}$ using $\mathrm{ADH}$ as a ligand to produce the drug carrier $\mathrm{GO} / \mathrm{CMC}$ complex, later characterized by infrared spectroscopy with infrared spectroscopy Fourier transform spectroscopy, thermogravimetric analysis (TGA), and transmission electron microscopy (TEM). Immediately after this, DOX, an antitumor medication, was loaded onto the surface of the conjugate by $\pi-\pi$ stacking interactions and hydrogen bonding. The in vitro release behavior under different $\mathrm{pH}$ conditions was monitored by spectroscopy analysis in the visible ultraviolet (UV-vis). This release confirmed that this drug carrier has a $\mathrm{pH}$ sensitivity, being able to carry anticancer drugs without reaching the vicinity of the carcinogenic cells, that is, healthy cells (Fig. 12). Therefore, the goal of targeted therapy is achieved. Cancer cells from the human cervix (Hela cells) and those from mouse fibroblasts (NIH-3T3 cells) were treated with the MTT method (3-(4,5-dimethylthiazol-2-yl)-2,5-diphenyltetrazolium bromide) (MTT). The results showed that GO/CMC did not present apparent cytotoxicity but good biocompatibility. In addition, GO/CMC/DOX offers excellent antitumor activity and is safer than the single administration of DOX. It can be concluded that the system improved the bioavailability of drugs and reduced the side effects in clinical application.

These results are aligned with those described by Jiao et al., who developed a drug administration system based on GO modified with methotrexate-loaded carboxymethylcellulose, a clinically used anticancer medicine [192]. The results showed good properties for releasing $\mathrm{pH}$-dependent drugs, reduced cytotoxicity against NIH-3T3 normal cells, high cytotoxicity against HT-29 cancer cells, higher plasma drug concentration, longer duration of action, 


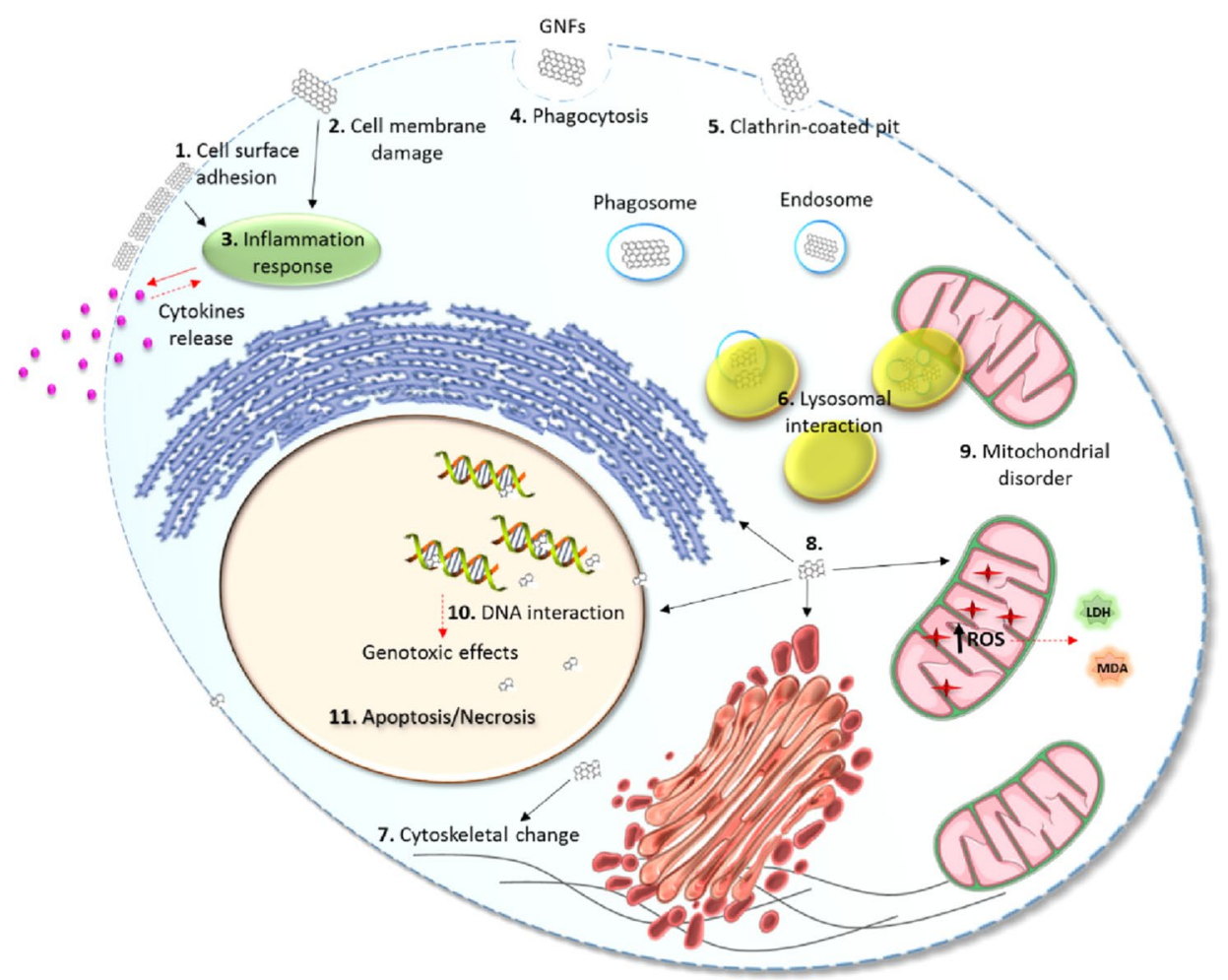

Fig. 11 Possible interactions of graphene family nanomaterials (GNFs) with the plasma membrane and cytotoxic effects. GNFs can interact with the plasma membrane through (1) adhesion to the outer surface of the cell membrane or (2) direct incorporation or translocation by the lipid bilayer of the plasma membrane. (3) The agglomeration and/or penetration of GNFs into the plasma membrane can damage it, inducing an inflammatory response due to the leakage of the cytoplasmic content. Additionally, GNFs can be internalized through (4) phagocytosis by specialized cells and/or (5) internalization by endocytosis mainly mediated by clathrin. Most of the graphene-based nanomaterials are internalized and directed in vesicles (phagosomes and endosomes) to the lysosomes where they are (6) degraded or sent to other cell compartments, however, a small part may remain free in the cytosol. In the cytosol, GNFs can (7) interact with components of the cytoskeleton and (8) with cellular organelles, including the mitochondria. (9) The GNF-mitochondria interaction can cause mitochondrial disorders, mainly by increasing the production of reactive oxygen species (ROS) and generating toxic effects such as increased lactate dehydrogenase ( $\mathrm{LDH})$ release due to reduced mitochondrial membrane potential and increased lipid peroxides, for example, malondialdehyde (MDA). (10) GNFs can also cross the nuclear membrane and interact directly with DNA, causing genotoxic effects and/ or (11) inducing cell death by apoptosis or necrosis in response to the mentioned cytotoxic effects. Source: authors

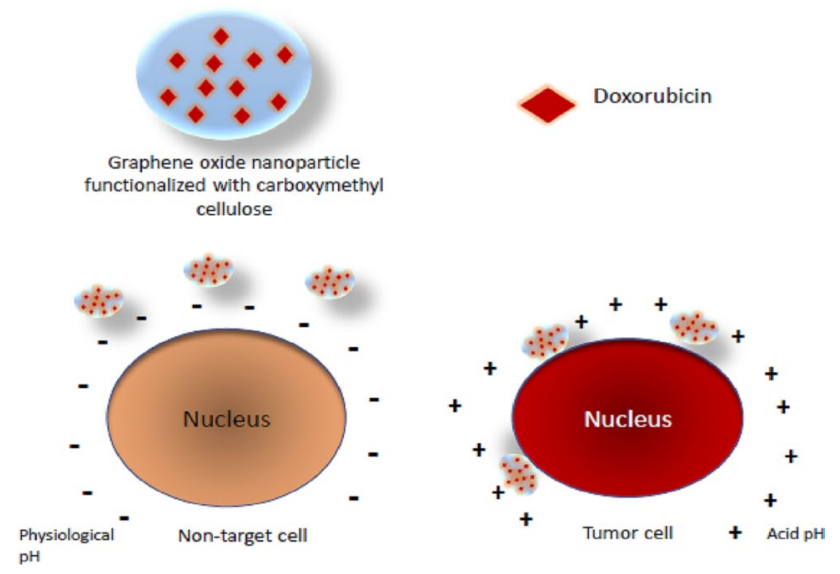

Fig. 12 Selective drug delivery scheme in response to changes in the cellular microenvironment. Source: authors superior tumor inhibitory activities, and inhibiting activities of liver metastasis. Additionally, the survival time of the rats was extended after the administration of MTX/ CMC/GO. Therefore, the MTX/CMC-GO drug delivery system has great potential in the treatment of colon cancer.

Javanbakht and Namazi also proved that DOX was efficiently loaded and released from the CMC/GO nanocomposite [193]. DOX-loaded nanocomposites have increased anticancer properties. In the microenvironment of tumor cells at $\mathrm{pH} 5$, the DOX release rate was significantly higher than under physiological conditions at $\mathrm{pH}$ 7.4. The MTT results showed that DOX/CMC/GO exhibits significant cytotoxicity in K562 cells, an erythroleukemic cell line. The resulting nanocomposite showed that this carrier system could potentially be used in cancer treatment drug delivery and delivery systems. 
Fan et al. developed a new anticancer system with controlled and targeted release skills [187]. Sodium alginate (SA) was covalently conjugated to $\mathrm{GO}$ by forming amide bonds, making it functional. The resulting GO/SA conjugate was characterized by FTIR, TGA, TEM and used as a vehicle to encapsulate DOX, an anticancer medicine chosen to study the release behavior and targeting under different $\mathrm{pH}$ conditions by UV-vis monitoring. The results showed that the nanocomposite exhibited a $\mathrm{pH}$-sensitive DOX release behavior with a faster rate and a more significant amount being released under acidic conditions. The cell viability test was applied to assess cytotoxicity using Hela and NIH$3 \mathrm{~T} 3$ cells. The results showed that GO/SA could specifically transport DOX to Hela cells with increased toxicity. At the same time, GO/SA exhibited extremely low cytotoxicity and no toxic effects evident in NIH-3T3 cells. These positive results suggested that $\mathrm{GO} / \mathrm{SA}$ is an ideal drug carrier for targeted and controlled drug delivery.

Dong et al. developed a medication administration system based on pegylated GO to load DOX [194]. The properties of the carrier, drug release behavior, cell uptake, and cytotoxicity were studied in vitro. They also explored the penetration of the functional transporter into the blood-brain barrier and its accumulation in the tumor region of rats with glioma. In addition, they evaluated the chemotherapeutic effects combined with treatment with GO/PEG/DOX in vivo and in vitro. Chemotherapy combined with GO/PEG/DOX prolonged the survival time of rats with glioma as compared with free DOX. The results of the in vitro and in vivo studies were promising. In conclusion, a potential nanoscale drug delivery system was developed for combined glioma therapy that can effectively decrease side effects and improve therapeutic effects.

These results were recently reaffirmed by Kazempour et al., who developed a new drug administration system [195]. It was made from the functionalization of the GO surface with PEG. The system was characterized with the scanning electron microscope (SEM), X-ray diffraction spectroscopy (XRD), and Fourier-transformed infrared spectroscopy (FTIR), and then DOX as an anticancer drug was immobilized on the GO-PEG surface. The release profile at two different $\mathrm{pH}$ levels and the cell viability assay were performed. The GO/PEG hybrid shows more release in acidic $\mathrm{pH}$, and according to the results of the cell viability test, it is more biocompatible than free GO. Hence, the proposed system has high potential as a drug delivery agent.

Chai also developed a new delivery system for the chemotherapy drugs oridonin and methotrexate (MTX) [196]. PEG was attached to the surface of GO sheets to improve its stability and biocompatibility. When starting the in vivo tests by the WST-8 assay, it was found that the GO/PEG conjugate has virtually no toxicity for normal human $293 \mathrm{~T}$ cells (human renal epithelial cells) and tumor cells of CAL27, MG63, and HepG2. Additionally, oridonin and MTX were combined with GO/PEG through $\pi-\pi$ stacking and hydrophobic interactions. The same tests showed that GO/PEG/oridonin and GO/PEG/MTX were more cytotoxic to tumor cells CAL27 (human tongue squamous cell carcinoma), MG63 (human osteosarcoma), and HepG2 (human liver tumor) than oridonin and methotrexate free, respectively. Therefore, GO/PEG could effectively improve solubility in aqueous media and increase the bioavailability of antitumor drugs to be a favorable and universal nanomaterial.

Recently, other researchers also developed a selective delivery system, and Borandeh et al. grafted $\beta$-cyclodextrin $(\beta-C D)$ into GO using L-phenylalanine (Phe) as a binder to improve stability, load capacity of the medication, and biocompatibility [197]. The GO/Phe/CD conjugate was characterized structurally and morphologically, and DOX was used as a chemotherapeutic model to examine drugs' loading and release properties. At the same time, the $\mathrm{pH}$ effect was also investigated. The GO/Phe/CD's efficiency and load capacity of DOX of the GO/Phe/CD was high, and the highest release rates were in an acid medium generated by the cancer cells. In addition, the overall cytotoxicity of the nanocarriers was examined by the MTT assay and exclusion of the trypan blue dye in the MCF-7 cell lines, where both tests demonstrated significant cytocompatibility. Thus, the GO/Phe/CD loaded with DOX had an excellent capacity to kill MCF-7 cells. The authors concluded that a drug delivery system responds to $\mathrm{pH}$, which may be a suitable candidate for the administration of chemotherapy drugs.

Furthermore, Prabakaran et al. developed a new medication administration system to reduce cytotoxicity in normal cells and increase the anticancer therapeutic effect through more excellent transporter permeability [198]. Here, poly(methyl methacrylate) (ovalbumin) and egg white protein, ovalbumin (OVA) was used to functionalize GO and DOX loaded into the drug delivery system. The formation of the OVA/PMMA and OVA/PMMA/GO hybrids were confirmed by their structure as visualized by different instrumental techniques. The cytotoxicity and in vitro release tests of the OX-PMMA and OVA-PMMA/GO conjugates loaded with DOX were investigated through the gastric cancer cell line and the standard cell line. In vitro studies showed that OVA/PMMA/GO has a pH-dependent release, and the most significant release occurs at acidic $\mathrm{pH}$. The in vitro drug release has also been proven by research using CACO-2 cancer cells, treated with OVA/PMMA/GO loaded with DOX, as OVA/PMMA/GO has the potential for more significant encapsulation/entrapment of the DOX drug, more great permeability, and more outstanding biocompatibility. All these characterizations in this study show that the drug is successfully loaded onto this new drug carrier, and controlled release has been achieved. 
Pourjavadi et al. also developed a new nanocarrier for drug delivery where a live polymerization method was used to engraft poly (glyceryl methacrylate) (PGMA) chains on the surface of GO [199]. The DOX was loaded covalently in the nanocarriers through stacking $\pi-\pi$. Then, the in vitro studies with two different $\mathrm{pH}$ (7.4 and 5.4) showed that the DOX-conjugated nanocarrier exhibits a $\mathrm{pH}$-dependent release behavior. Cytotoxicity and MTT studies have demonstrated excellent biocompatibility. Cell capture studies have shown that the DOX-conjugated nanocarrier can be very efficient for delivering and releasing DOX in HeLa cells. The results showed that this nanocarrier has good potential application as an anticancer drug delivery system.

Deb et al. developed a nanocomposite from the functionalization of GO with PEG decorated with folic acid (FA) for the co-delivery of two anticancer drugs, camptothecin (CPT) and 3,3'-Diindolylmethane (DIM) [200]. The difference in the mechanism of action of the two drugs makes them promising candidates to produce a synergistic effect against breast cancer. The data showed a significant increase in anticancer activity and a delayed and controlled release of drugs. In vivo studies have also shown that DIM has successfully masked the toxic effects produced by CPT. In general, the combinatorial effect produced by the nanocomposite loaded with CPT-DIM could serve as a promising chemotherapeutic agent against breast cancer with greater bioavailability and fewer side effects.

Pourjavadi et al. also developed a new transporter based on modified polyglycerol-branched GO for the co-administration of anticancer drugs, curcumin (CUR) and DOX loaded through the $\pi-\pi$ stack and covalent bond, respectively [201]. Based on the results of the cell viability assay, the carrier showed low toxicity to normal cells. For cancer cells, MCF-7, the cytotoxicity was more significant than that of the carrier loaded with only one of the drugs. It was also observed that the release of both drugs was sensitive to $\mathrm{pH}$, with effective release to acidic $\mathrm{pH}$. There was greater internalization of the drug co-administered compared with the only vehicle loaded with the drug.

More recently, Pei et al. also developed a dual drug delivery system using GO functionalized with PEG (pGO) and cisplatin $(\mathrm{Pt})$, and DOX antitumor drugs were incorporated through amino linkage and noncovalent reaction through $\pi-\pi$ interactions, respectively [202]. The advantage of this dual drug delivery system was shown by detecting its effectiveness, drug release, and cytotoxicity against cancer cells in vitro and in vivo. In vitro studies showed that $\mathrm{pGOq} / \mathrm{Pt} /$ DOX was effectively delivered to tumor cells, indicating more prominent apoptosis and cell necrosis, and then exhibited a higher growth-inhibiting property than the single drug or free drug delivery system. In vivo data confirmed that the pGO-Pt/DOX dual drug delivery system had attenuated toxicity to normal organs and a more effective anticancer effect than free drugs. Additionally, the results of drug release indicated the release of drugs in an acidic environment. As a result, the authors argue that this system has great potential for clinical applications.

In determining the selectivity to acid environments of GO nanocarriers, Gao et al. conducted a study to evaluate the impact of GO on the activity against three Gram-positive (Staphylococcus aureus) and Gram-negative (Escherichia coli) bacteria of three conventional antibiotics: lincomycin hydrochloride (LMH), chloramphenicol (CPC), and gentamicin sulfate (GMS) [203]. These antibiotics were chosen because they are common and used uncontrollably. In addition, these antibiotics demonstrate an affinity for adsorption with GO, and the sensitivity of Gram-negative and Gramnegative bacteria is different. GO was functionalized with PEG and antibiotics adsorbed to it. The effect on the antibacterial activity of the free and combined antibiotic was studied. The results showed a remarkable impact on the efficiency of the antibacterial activity concerning the free antibiotic. However, they emphasized the challenge of building antimicrobial agents based on the drug delivery system using graphene and the need for more study to fully understand the mechanisms of toxicity and cellular responses to the combination of GO and antibiotics.

These findings were reaffirmed by Prabakaran et al., who developed a drug delivery system using GO functionalized with PEG and iron oxide to assess the antibacterial activity against the Gram-positive strain (S. aureus) and Gram-negative strain (E. coli) [198]. The results showed an inhibitory effect against bacterial strains in a concentration-dependent manner: the higher is the concentration, the greater is the antibacterial activity. They also demonstrated a more significant antibacterial activity than antibiotics adsorbed to GO.

Considering these advances, Katuwavila et al. developed a sustained-release transport system for the cephalexin drug (CEF) using GO functionalized with PEG [204]. The results demonstrated that the sustained release of the CEF was achieved. Additionally, the GO/PEG/CEF compound showed improved antibacterial activity as compared with the positive control in gram-positive bacteria. Developing a new controlled release system for CEF would result in a higher concentration of the antimicrobial agent at the infection site, better patient compliance with treatment, and more constant blood levels. In addition, the need for high doses of antibiotics to achieve the therapeutic effect can be avoided through controlled release.

Saifullah et al. developed an anti-tuberculosis formulation using pegylated GO and ethambutol as a drug carried through adsorption. It was found that the sustained release of the drug resulted in better bioavailability [205]. In addition, the designed formulation demonstrated high biocompatibility with mouse fibroblast cells. The results also showed that ethambutol's antimycobacterial activity was not affected by 
the drug's loading and release process. In this way, therapy with reduced dosage frequency would be feasible, reducing the associated side effects and increasing the patient's adherence to treatment. In conclusion, the results of this study demonstrated the potential of this new formulation strategy to produce a more effective antibiotic therapy against tuberculosis.

Karimzadeh et al. developed a drug delivery system from graphene modified with carboxymethylcellulose and a metal-organic structure based on zinc (CMC/MOF5/GO) loaded with tetracycline, an antibiotic used to treat a series of infections [206]. Antibacterial activity tests showed that TC/CMC/MOF-5/GO has a more significant and selective antibacterial activity than free tetracycline confirming its potential for targeted delivery and controlled release of oral delivery.

Yang et al. also showed that angiogenesis is essential in cancer initiation, progression, and metastasis. At that moment, there is overexpression of the follicle-stimulating hormone receptor (FSHR) [207]. They developed a nanocarrier for selective drug administration. At the same time, GO was conjugated to a monoclonal antibody $(\mathrm{mAb})$ selective to the FSHR overexpression and Copper-64 incorporated as a radiomarked, thus allowing these visualization nanodevices using positron emission tomography (PET) images. The lung metastasis model for breast cancer was established by intravenous injection of breast cancer cells MDA-MB-231, and tumor growth was monitored. Systematic in vitro and in vivo studies were carried out to investigate the stability, aiming at the nanocarrier's effectiveness, specificity, and tissue distribution. Examination by flow cytometry and fluorescence microscopy confirmed the specificity of targeting. A more potent and persistent uptake in nodules within the lung was observed compared with nanocarriers not directed towards the FSHR overexpression. The histological evaluation also confirmed the nanocarrier accumulation in the tumor vasculature in early moments; that is, they were captured not specifically in the liver and spleen.

Further, these nanocarriers can serve as suitable drug carriers, for example, for DOX. Improved efficiency of DOX administration in metastatic sites has been demonstrated by fluorescence imaging. GO has proven to be a valuable tool for early detection of metastases and targeted delivery of therapy.

These results are in line with the findings of Pan et al., who carried out a study in which a drug administration system was built by the functionalization of GO with carboxymethyl chitosan (CMQ), fluorescein isothiocyanate, and lactobionic acid (LA), with DOX being carried in the composites by adsorption [184]. The resulting formulations have a high charge content and efficiency (>96\%), $\mathrm{pH}$-sensitive release, and selectively induced the death of cancerous liver cells but were not toxic to a non-cancerous cell line. Based on the in vivo tests, it is concluded that the induction of death in a particular way occurred due to the selective recognition of LA by the asialoglycoprotein receptors. These receptors are overexpressed in the cancerous liver cells, where the free GO compounds were not cytotoxic, but the systems loaded with DOX are effective in inducing cell death, being almost as potent as the free drug. These results are promising, with great potential for targeted anticancer therapy in vivo.

More recently, Bullo et al. developed a new GO nanocarrier functionalized with poly (ethylene glycol) (GO/PEG), loaded with two anticancer drugs: Protocatechuic acid (PCA) and chlorogenic acid (CA) [208]. The anticancer nanocomposite was also coated with folic acid, a specific targeting agent for cancer since cancer cells are overexpressed with folate receptors compared with normal cells (Fig. 13). Anticancer properties have been evaluated against liver cancer cells (HEPG2) and human colon cells (HT-29). Cytocompatibility was assessed in normal 3T3 fibroblast cells. The in vitro release of the PCA and CA drugs occurred sustainably, which took more than $100 \mathrm{~h}$ for release. Additionally, the designed formulation was biocompatible with normal 3T3 cells and showed a better anticancer effect than free drugs.

These results align with Deb, who focused on achieving targeted delivery of drugs using CPT [200]. GO nanoparticles functionalized with PEG were also decorated with FA and used to increase the efficiency and specificity of drug uptake by cancer cells. Then, the CPT was added to pegylated GO. As seen by UV analysis, the GO/PEG/FA/ CPT drug delivery system showed a $\mathrm{pH}$-dependent drug
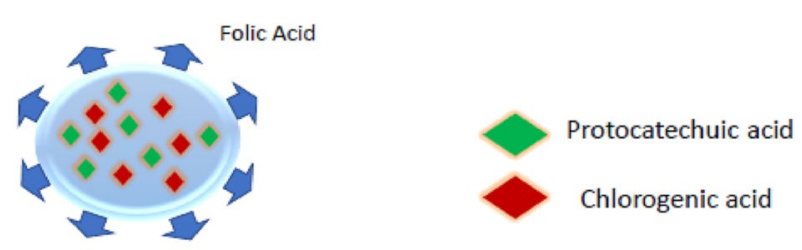

Graphene oxide nanoparticle functionalized with coated polyethylene glycol folic acid
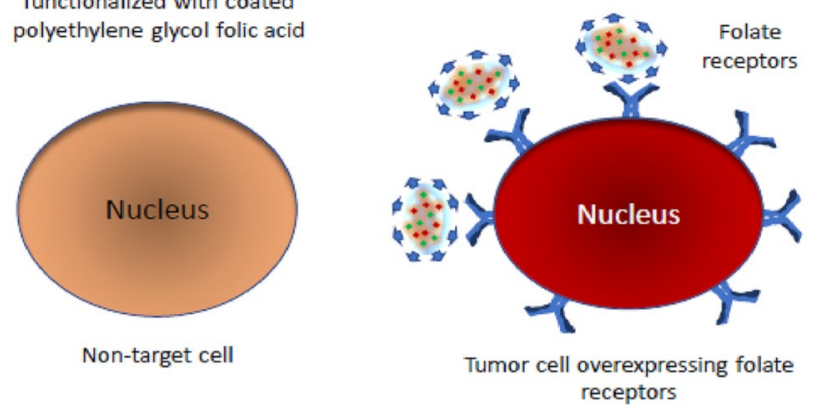

Fig. 13 Endocytosis scheme of PCA and CA-containing graphene oxide nanoparticles functionalized with PEG and coated with folic acid. Source: authors 
release. In addition, the non-toxic nature was confirmed by the cell viability assay.

\section{Graphene quantum dots (GQDs)}

Graphene quantum dots (GQDs) have also become materials of interest for drug delivery, as the unique electronic structure of GQDs gives functional attributes to these nanomaterials promoting high load capacity [209-212].

As demonstrated by De et al., the graphene oxide quantum dot (GOQD) conjugated to chitosan functionalized with folic acid (FA/CH) efficiently carries and delivers the anticancer drug DOX to the cancerous tissue [213]. As a result, the in vitro release pattern was examined in several $\mathrm{pH}$ ranges, where the drug release rate at $\mathrm{pH} 5.5$ was higher than in the physiological range of $\mathrm{pH} 6.5$ and 7.4. Additionally, cell uptake studies have shown that FA/CH/GOQD specifically transported DOX to cancer cells by overexpressing folate receptors. Following this direction, Ko et al. [214] also developed nanoparticles based on GQDs conjugated to poly(ethylene glycol)-disulfide-Herceptin that increased the half-life and conferred the ability to target with DOX. The in vitro analysis confirmed the successful internalization in the cell cytoplasm and the increase in the uptake and therapeutic action of DOX in HER2-positive breast cancer cells.

According to Flak et al., the therapeutic activity of DOX became more efficient through delivery directed by GQDs, where they did not present toxicity against non-target cells and $\mathrm{pH}$-dependent drug release [215]. In another study conducted by Yang et al., a new nanocarrier for pancreatic cancer has been composed of biodegradable polyester-based vectors (BCPVs) and GQDs, demonstrated high stability in physiological media and release in acid medium, and high capacity of a load of the anticancer drug DOX resulting in effective inhibition of the pancreatic cancer cell bioactivity (MiaPaCa-2 cells) [216]. Additionally, Rakhshaei et al. synthesized a nanocarrier with high biocompatibility and high potential to be used as a specific drug delivery system driven by acid $\mathrm{pH}$. Still, they functionalized the GQDs with carboxymethylcellulose and used DOX as an anticancer medicine [217]. The cytotoxicity of the nano-transporter was studied using HT29 cells from human colon adenocarcinoma.

Furthermore, Sui et al. demonstrated the application of GQDs by combining it with cisplatin (CDDP), a drug with antitumor activity attributed to DNA binding [218]. It was shown a significant increase in cell uptake, improving cell permeability and reinforcing its interaction with DNA once inside cells, thus increasing cytotoxicity, cell cycle arrest, and DNA fragmentation, which can increase the effectiveness of drug chemotherapy anticancer.

This idea was reinforced by Wei et al., as they developed a nanocomposite of GQDs functionalized with poly (ethylene glycol) to load cisplatin (GPt) [212]. This result caused the cell cycle to stop leading to apoptosis and increased accumulation of intracellular cisplatin. Therefore, GPt proved to be a promising candidate for anticancer therapy, as it improves the drug's pharmacokinetics, promoting more significant accumulation in the tumor and minimizing systemic toxicity.

Finally, Sheng et al. developed GQDs functionalized with chitosan gels (CS) for loading hydrophilic cytarabine (Cyt), an anticancer drug [219]. The release of Cyt from the designed carrier was sensitive to acidic $\mathrm{pH}$. The incorporation of Cyt in GQDs was achieved by an amidation reaction hydrolyzed in an acid medium.

Ko et al., in their study, developed a drug delivery system for breast cancer with overexpression of the human epidermal growth factor (HER2) type 2 receptor [220]. A new graphene-based nanocarrier (GQD) marked with Herceptin (HER), a drug selective for the biomarker, and $\beta$-CD for particular DOX delivery has been developed as a promising theranostic candidate for the treatment of breast cancer with HER2 overexpression. In response to the acidic environment of cancer cells, the GQD complex was rapidly degraded, and DOX was released in a controlled manner to inhibit cancer cell proliferation. Nanocarriers also provide diagnostic effects due to the blue color emission from GQDs. This multifunctional drug delivery system leads to a synergistically enhanced anticancer strategy that supplies treatment and diagnosis. The theranostic application of GQDs has also been reported by Song et al. [221]. Before that, it is essential to know the toxicity of graphene-based nanostructures. Thus, Table 4 summarizes the main information from toxicological studies of the graphene family nanomaterials.

\section{Application in photothermal application}

Despite the numerous nanomaterials studied and applied in photothermal therapy (PTT), graphene is the newest one [222]. PTT involves an increase in local temperature after exposure of a photothermal agent to electromagnetic radiation, such as visible light or NIR (Near Infra-Red), inducing tumor cell death by converting light energy into heat [223]. Graphene and its derivatives are excellent photothermal agents used for PTT due to their multifunctionality and high photothermal conversion capacity. Robison et al., a pioneer in using $\mathrm{rGO}$ for PTT, demonstrated that nanometric rGO produced by chemical reduction of GO has a NIR absorption rate six times higher than GO, endorsing its preferential use over GO as a photothermal agent [224]. Furthermore, combining graphene-based materials with inorganic particles such as gold nanoparticles can further increase the photothermal effect and lead to a higher rate of tumor cell death during PTT [225]. The rGO photothermal applications have been explored with other nanoparticles and photosensitizers in synergy for tumor therapies. Otari et al. performed the one-step reduction of GO to rGO. They decorated the 


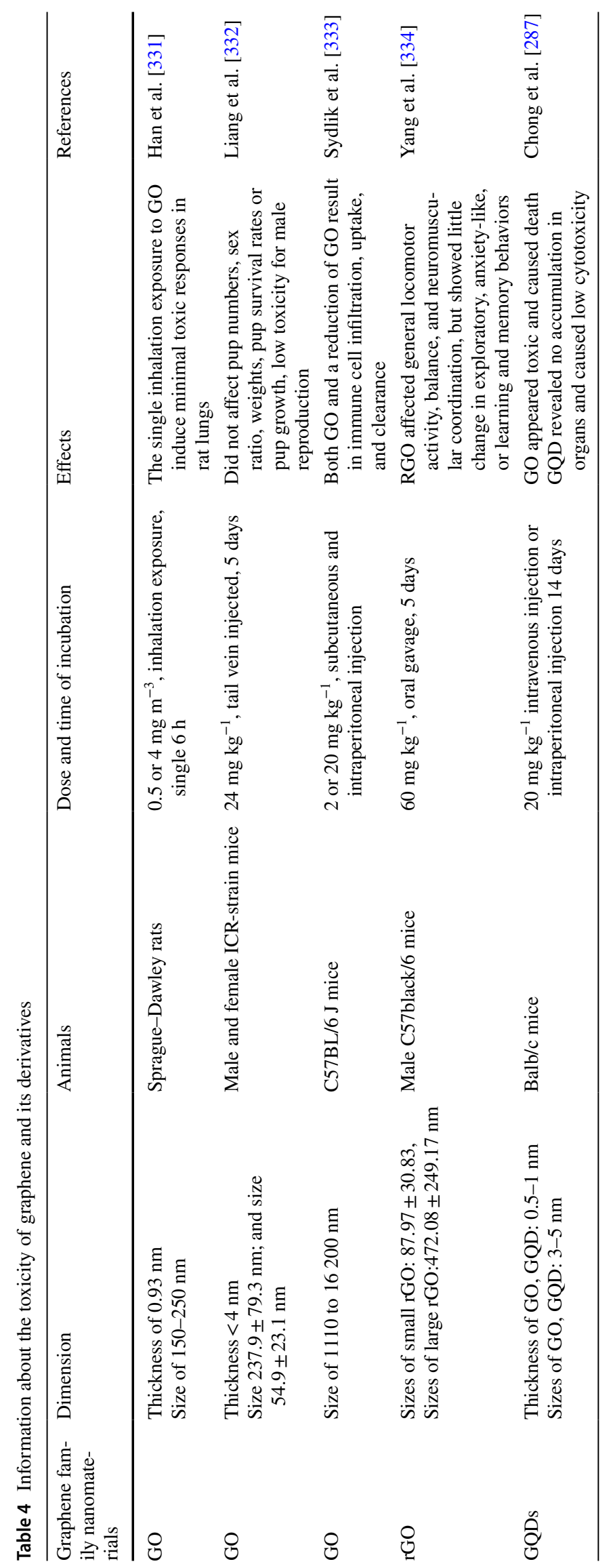


rGO with gold nanoparticles (NAuNPs) using thermostable nisin antimicrobial peptides to form a nanocomposite of reduced graphene oxide (NAu-rGO). High-resolution transmission electron microscopy (HR-TEM) analysis revealed the formation of spherical AuNPs 5-30 nm in size in rGO nanosheets. After treating MCF-7 breast cancer cells with the nanocomposite followed by treatment with $800 \mathrm{~nm}$ diode laser $(0.5 \mathrm{~W} / \mathrm{cm} 2)$ for $5 \mathrm{~min}, 80 \%$ cell growth inhibition was found [225].

More recently, Chen et al. developed a PTT to treat cancer using Alanine-functionalized GO (ARGO) through $\pi-\pi$ interactions. The conjugation between alanine and GO increased by twofold the stability of ARGO compared to rGO in aqueous solution and greatly improved the absorption of NIR radiation, resulting in increased photothermal conversion efficiency when irradiated with an $808 \mathrm{~nm}$ laser. Using $808 \mathrm{~nm}$ laser irradiation, PTT produced an $89 \%$ and $33 \%$ greater photothermal effect than GO and rGO isolated, respectively, and acted as a targeting ligand to selectively kill U87MG tumor cells. Thus, this ARGO platform composed of active functional groups allows precise targeting in PTT without damaging healthy tissues and cells [226].

Another form of therapy involving light and a photosensitizer is photodynamic therapy (PDT). PDT consists of photochemical reactions associated with a photosensitizing substance and a light source, which in conjunction with oxygen can produce molecular oxygen or reactive oxygen species inducing tumor cell death [222]. Studies showed that the combination of PTT and PDT is promising to cause tumor cell death more efficiently compared to PTT or PDT alone [227-229]. Phototherapeutic effects of PTT and PDT combined using graphene and its derivatives as cancer treatments have shown surprising results. Zhang et al. developed an rGO-Ru-PE nanohybrid through hydrophobic $\pi-\pi$ interaction of rGO sheets and a Ru (II) complex modified with phosphorescent polyethylene glycol (Ru-PEG). They applied it as a photothermal agent and a photosensitizer for PTT/ PDT. And A549 lung cancer cells, sequentially exposed to $808 \mathrm{~nm}$ (for PTT) and $450 \mathrm{~nm}$ (for PDT) wave light source. The results showed increased cytotoxicity because of the combined effect of the two therapies [227].

Interestingly, Jiang et al. created a nanoplatform using dopamine-reduced GO nanosheets (rGO-PDA) loaded with chlorin e6 (Ce6) to increase photothermal capacity. The proposed nanoplatform was successfully used to precisely deliver the photosensitizer chlorin e6 (Ce6) into the tumor cells, especially those that overexpressed CD-44. Combining excellent NIR photothermal conversion capability and controllable $\mathrm{Ce} 6$ release gives the nanoplatform enhanced singlet oxygen generation, giving higher cytotoxicity [228]. Similarly, Shim et al. designed a claudin 4 driven dual photodynamic and photothermal system in which Clostridium perfringens enterotoxin (CPE) was linked to the photodynamic agent $\mathrm{Ce} 6$ and anchored in rGO. This nanosystem was intracellularly delivered in U87 glioblastoma cells. The combined therapy was performed using $660 \mathrm{~nm}$ light source for the PDT and $808 \mathrm{~nm}$ for PPT [229].

Recently, photoimmunotherapy has been used for the treatment of metastatic tumors [230-232]. Wang et al. developed a PEGylated nanocomposite through the hybridization of $\mathrm{Fe} 3 \mathrm{O} 4$ nanoparticles (FNPs) with rGO through electrostatic interaction for photothermal immunotherapy. The results in vivo revealed that FNPs/rGO-PEG nanocomposites when combined and irradiated by an $805 \mathrm{~nm}$ laser, could destroy primary tumors. Furthermore, intratumoral injection of FNPs/rGO-PEG nanocomposites in animals inducted with breast tumors and irradiated with near-infrared laser irradiation showed a reduction in tumor volume [230]. Corroborating these findings, a new treatment strategy for metastatic cancer through the synergy of photothermal therapy (PTT), chemotherapy, and immunotherapy using a nanosystem to trigger the host's antitumor immunity was also developed by Zhou et al. The nanosystem was constructed by loading the chemotherapeutic agent mitoxantrone (MTX) and the TGF- $\beta$ inhibitor SB-431542 (SB) in rGO. Intratumoral administration of $\mathrm{rGO} / \mathrm{MTX} / \mathrm{SB}$, followed by non-invasive irradiation of a near-infrared laser, destroyed local primary tumors and inhibited distant metastases, as demonstrated in the murine model. After treatment, $70 \%$ of tumor-bearing mice became long-term survivors and developed specific immunity with increased infiltration of tumor-specific CD8 + cytotoxic $\mathrm{T}$ lymphocytes and decreased infiltration of regulatory $\mathrm{T}$ cells (Tregs) into distal tumors. Thus, the administration of rGO/MTX/SB via local injection combined with laser irradiation provided a synergistic chemo-immunophotothermal effect against tumors with inhibition of the immunosuppressive microenvironment [231].

Finally, multiple combination immunotherapy, which included photothermal therapy (PTT), inhibition of indoleamine-2,3-dioxygenase (IDO), and blockade of programmed cell death ligand 1 (PD-L1), was developed to induce synergistic antitumor immunity. Yan et al. designed rGO nanosheets loaded with a multifunctional IDO inhibitor (IDOi) with the properties of directly killing tumor cells under laser irradiation and triggering the antitumor immune response in situ. The group demonstrated that the three combinations of PTT, IDO inhibition, and PD-L1 blockade could effectively inhibit the growth of irradiated tumors and tumors at distant sites without PTT treatment [232].

\section{Imaging-related applications}

As previously mentioned in this review, graphene and its derivatives have intrinsic fluorescence properties, making them bioimaging agents of cancer cells; these properties are called GQDs, making these fluorescent materials of great 
interest today [220, 233-239]. Just as the functionalization of graphene proved to be an essential step, the functionalization of the GQDs guarantees the viability of its use in biological applications linked to its intrinsic fluorescence property [209-211, 240, 241].

Obtaining GQDs through top-down and bottom-up approaches leads to a significant difference in the intrinsic properties of the resulting product, such as size, layer, shape, or edge configuration, which eventually results in photoluminescence (PL) diversity the final GQDs [242]. The absorption spectrum of GQDs in the UV region is in the range varying from 4.6 to $6.2 \mathrm{eV}$, with a sharp peak at $260 \mathrm{~nm}$ and a tail in the visible region up to $2.1 \mathrm{eV}$, these are attributed to pi-pi excitations $(\pi \rightarrow \pi *)$ from the $\mathrm{C}=\mathrm{C}$ bonds and the excitation $\mathrm{n} \rightarrow \pi *$ from the $\mathrm{C}=\mathrm{O}$ bonds, respectively [243, 244].

The main difference between conventional QDs and GQDs is the PL bandwidth. Unlike traditional quantum dots (QDs), which do not exhibit luminescence behavior for nonzero band gaps, GQDs have a non-zero gap and excellent luminescence behavior under excitation [245]. The PL band spectra of GQDs are vast and change with the excitation wavelength ranging from 300 to $470 \mathrm{~nm}$. The spectrum of the PL band can be assigned due to the transition from the lowest unoccupied molecular orbital (LUMO) to the highest occupied molecular orbital (HOMO) [244]. In this sense, GQDs with larger diameters have smaller band gaps and vice versa. According to Sk et al., GQDs with diameters ranging from 0.46 to $2.31 \mathrm{~nm}$ emit deep UV fluorescence to the nearinfrared (NIR) regions. In comparison, GQDs with diameters ranging from 0.89 to $1.80 \mathrm{~nm}$ emit fluorescence covering the entire visible light spectrum range $(400-770 \mathrm{~nm})$ [246]. The NIR fluorescence of GQDs showed excellent performance due to their remarkable attributes such as deep penetration depth, unblinking fluorescence emission, easy spatiotemporal control, good water solubility, and biocompatibility [247, 248].

\section{Near-Infrared (NIR) GQDs fluorescence}

In recent years, fluorescence imaging of biological systems in the second near-infrared window (NIR-II) has drawn much attention due to their negligible autofluorescence background noise and low tissue scattering [245]. Wang et al. developed dual-doped nitrogen and boron GQDs (N-BGQDs) that served as NIR-II contrast agents and investigated in vivo imaging capability. The N-B-GQDs had an ultrasmall size $(\sim 5 \mathrm{~nm})$, high stability in serum, fluorescent emission peak at $1000 \mathrm{~nm}$, and high photostability. Furthermore, in addition to the NIR-II imaging capability, NB-GQDs absorb and convert NIR light to heat when irradiated by an external NIR source, demonstrating a photothermal therapeutic effect that kills tumor cells in vitro and completely suppresses tumor growth in a glioma xenografted mouse [249]

One of the main issues related to cancer is early detection. The detection of cancer in the early stages increases the probability of the patient's recovery, so the necessity of a more sensitive fluorescent probe is required [238, 250, 251]. In this direction, the use of GQDs as fluorescent probes has been reported by Badrigilan et al., which have exemplified the application of GQDs in the generation of high-performance images by computed tomography [252]. They synthesized bismuth nanoparticles coated with GQDs, and these exhibited good physiological dispersion with blood compatibility and satisfactory cytotoxicity, in addition to offering a solid and constant absorbance profile in near-infrared spectroscopy with excellent photostability.

\section{Modified GQDs for fluorescence and magnetic resonance images}

Regarding biomedical application, Wang et al. demonstrated that pegylated GQDs are suitable for biomedical imaging [253]. As a result, the quantum dots of graphene were functionalized with PEG by the hydrothermal method. Subsequently, the cell viability test results showed that the GQDs-PEG does not exhibit apparent toxicity to the cells. The results of the fluorescence images showed high recognition of GQDs-PEG. Nonetheless, Feng et al. synthesized nitrogen-doped GQDs reacted with indomethacin [254]. This fluorescent probe was then incubated with breast cancer cells (MCF-7) for imaging through a Laser Scanning Confocal Microscope. This compound acted as a fluorescent probe material that rapidly recognized MCF-7 cells within a few hours. It was also noted that the fluorescence intensity increased as the incubation time was extended. Therefore, this compound can be suggested as a promising fluorescent probe for recognizing and labeling cancer cells by laser scanning confocal microscopy. Another study conducted by Campbel et al. demonstrated the excellent applicability of GQDs doped with nitrogen [255]. Nitrogen-doped GQDs exhibited a pH-dependent fluorescence response that is successfully used as a detection mechanism for acidic extracellular environments of cancer cells, allowing differentiation between cancerous environments (HeLa and MCF-7 cells) versus healthy environments (HEK-293 cells). They also had high biocompatibility, effective cell internalization, and emission in near-infrared spectroscopy.

Finally, in a study, Feng et al. demonstrated that the quantum dots of nitrogen-doped graphene reacted with FA to form FA/N/GQDs used for biological imaging, establishing a basis for the preparation of diagnostic materials for cancer [238]. FA can bind to folate receptors overexpressed in MCF-7 cells, and nitrogen-doped GQDs emitted blue fluorescence so that the recognition of cancerous cells was 
carried out. The authors also observed that the increase in the nitrogen content in GQDs provided more binding sites for folic acid, increasing the amount of FA/N/GQDs that enters the cell, improving the recognition of cancer cells, and more accurate diagnosis of cancer by fluorescence. Finally, laser scanning confocal microscopy images showed that FAN-GQDs had strong fluorescence stability for cancer cells over a period, which was more conducive to recognizing cancer cells.

\section{Fluorescence resonance energy transfer (FRET)}

These results are in line with those of Su et al., where the graphene quantum dots terminated in carboxyl (C-GQDs) were conjugated with iron oxide $\left(\mathrm{Fe}_{3} \mathrm{O}_{4}\right)$ and silicon dioxide and then functionalized with FA [256]. After that, the DOX molecules were loaded onto the GQDs surface through $\pi-\pi$ stacking, which resulted in $\mathrm{Fe}_{3} \mathrm{O}_{4} / \mathrm{SiO}_{2} / \mathrm{GQDs} / \mathrm{FA} / \mathrm{DOX}$ conjugates. The nanosystem underwent a viability study, magnetic resonance characterization, and in vitro fluorescence of Hela cells tests. The intracellular drug release process was monitored through fluorescence resonance energy transfer (FRET). The nanosystem proved to be efficient in the generation of imaging by fluorescence and magnetic resonance, aiming at precise diagnosis and detecting drug release based on FRET. It also demonstrated low cytotoxicity and, thus, an exemplary system for diagnosis.

\section{Single-photon emission computed tomography (SPECT)}

In terms of imaging, a study from Wang et al. endorsed the idea once they synthesized GQDs co-functionalized with PEG and FA to improve the capacity for biocompatibility and to target tumors, respectively [257]. Subsequently, the nanocomposites were radiolabeled with I-131 to assess biological behavior. The in vitro results demonstrated low cytotoxicity, high biocompatibility, and good stability. At the same time, the in vivo studies showed that images could examine the tumor through computed tomography by singlephoton emission (SPECT). They also demonstrate a more significant effect of permeability, retention, and active targeting to folate receptors. The uptake of 131I-GQDs-PEGFA at tumor sites can be clearly examined using SPECT images, which can attribute a more excellent permeability and retention effect and active targeting effect of $\mathrm{AF}$ to folate receptors. Also, Nasrollahi et al. developed an image nanostructure based on ferritin protein (AfFtn-AA) nanocages that encapsulate the GQDs and iron $(\mathrm{Fe})$ for fluorescence and magnetic resonance images [258]. It was then demonstrated that the non-target cells were underperforming. At the same time, the contrast in the magnetic resonance imaging (MRI) increased significantly, and the MDA-MB-231 breast cancer cells were imaged selectively since the fluorescence occurs after the release of Nanocages' GQDs in response to the acid environment. The researchers also carried out tests that emphasized the high carrying capacity of the nanosystem, demonstrating its promising application as a bimodal agent in the diagnosis and therapy of cancer. In another study reported by Zhang et al. developed a nanocomposite with diagnostic functionality using the apurinic/apyrimidinic endonuclease 1 (APE1) enzyme as a biomarker, as it is overexpressed in most human cancers [259]. This nanocomposite originated from the unification through adsorption of GQDs and unimolecular DNA architectures acting as a ligand to APE1. After using these nanocomposites as diagnostic probes, accumulation in the target cells was proven through the fluorescence observed selectively in response to APE1 overexpression, without cytotoxicity to neighboring cells. It was also highlighted that these nanocomposites could detect the cancer biomarker APE1 in identical types of cells under different cell conditions and can be applied to various cancer cells in extremely sensitive and specific ways.

\section{Theranostic applications}

Currently, there are a growing interest in developing nanomaterials for the simultaneous treatment and screening of cancer cells, that is, theranostic nanomaterials [216, 234, 239, 260-262]. Theranostic medicine combines diagnosis and treatment in the same procedure; it is a nanotechnological strategy that involves treatment with drugs carried by nanoparticles to target cells and monitoring of the response of therapy simultaneously [216, 239, 261, 263].

Graphene-based nanomaterials can act as agents for developing theranostic platforms to perform the most efficient detection and treatment of cancers [235, 239, 260-262]. In this direction, Chang et al. developed an attractive theranostic agent for efficient cancer diagnosis and treatment [264]. As a result, they cultivated manganese tungsten oxide nanoparticles $\left(\mathrm{MnWO}_{4} \mathrm{NPs}\right)$ in situ on GO surfaces in a polyether hyperthermia medium containing PEG to produce the hydrophilic nanocomposite $\mathrm{GO} / \mathrm{MnWO}_{4} / \mathrm{PEG}$. By comparison, it was observed that the absorbance in the near-infrared (NIR) of the $\mathrm{GO} / \mathrm{MnWO}_{4} / \mathrm{PEG}$ nanocomposite produced is significantly improved. The in vivo magnetic resonance and the generated photoacoustic image indicated that the nanocomposite could serve as an efficient bimodal contrast agent to guide cancer treatment. The therapeutic agent DOX was then loaded into $\mathrm{GO} / \mathrm{MnWO}_{4} / \mathrm{PEG}$ through $\pi-\pi$ interactions and electrostatic forces. GO/MnWO $/ \mathrm{PEG}$ demonstrated a high load capacity of DOX and drug release triggered by acidic $\mathrm{pH}$, without the appearance of toxic effects and apparent side effects. Chemotherapy combined with $\mathrm{GO} / \mathrm{MnWO}_{4} / \mathrm{PEG} / \mathrm{DOX}$ therapy injected intravenously can eliminate $4 \mathrm{~T} 1$ tumors, indicative of an excellent antitumor effect in vivo. 
The results were reaffirmed by Qian et al., who synthesized graphene-manganese oxide nanocomposites (RGO/ $\mathrm{MnFe}_{2} \mathrm{O}_{4}$ ) reduced through a hydrothermal reaction and functionalized with PEG [265]. The results showed that the nanocomposites resulted in an excellent magnetic resonance. After additional marking by Iodine- 125 radioisotopes, the single-photon emission computed tomography (SPECT) image exhibited a high accumulation of the nanocomposite in the tumor. It was also observed that radioisotope therapy and combined chemotherapy, performed using the nanocomposite-loaded DOX drug, significantly inhibited the growth of the 4T1 tumor after intravenous injection.

Recently, Gonzalez-Rodriguez et al. improved the functionality of GO with superparamagnetic iron oxide nanoparticles $\left(\mathrm{NPsFe}_{3} \mathrm{O}_{4}\right.$ ), which served as biocompatible magnetic additives for drug administration and magnetic resonance contrast agents [266]. The synthesized GO/ $\mathrm{Fe}_{3} \mathrm{O}_{4}$ nanoparticles showed low cytotoxicity to healthy cells (HEK-293). Then, DOX was loaded through $\pi-\pi$ stacking interactions and hydrogen bonding, and its imaging capability, cancer detection, and in vitro anticancer drug delivery in HeLa, MCF-7, and HEK-293 cells were tested. The nanoparticles showed intracellular delivery of DOX 2.5 times better when compared with free drugs in low concentrations (Fig. 14). This, in turn, allows the use of an eightfold lower dose of DOX to achieve the same therapeutic effect of approximately $62 \%$ cancer cell death. The dependence on $\mathrm{pH}$ allowed the differentiation between extracellular cancerous environments (MCF-7 and HeLa) and healthy ones (HEK-293). Finally, it was proposed that the $\mathrm{GO} / \mathrm{Fe}_{3} \mathrm{O}_{4}$ nanoparticles can act in delivering highly effective drugs, dose tracking and diagnosis

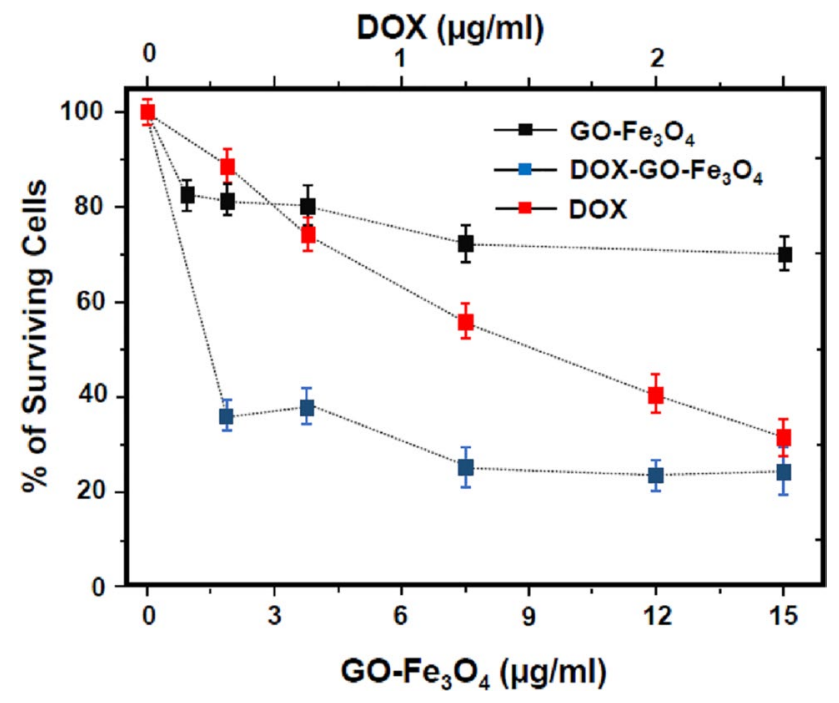

Fig. 14 Cell viability of $\mathrm{HeLa}$ cells subject to: $\mathrm{GO}^{-}-\mathrm{Fe}_{3} \mathrm{O}_{4}$ (black squares), DOX-go- $\mathrm{Fe}_{3} \mathrm{O}_{4}$ (blue squares), and dox (red squares). Adapted from Wei et al. 2018 [212] through magnetic resonance imaging. In this direction, Zhang et al. (2016b) obtained a nanocomposite thermoset from the preparation of nanograft-oxide oxide (NGO/ PEG), which co-administered paclitaxel (PTX) and green indocyanine (ICG), a fluorescent dye approved by the Food and Drug Administration (FDA) [267]. The fluorescence signal granted by the ICG was detected in the cytoplasm by confocal microscopy, demonstrating the high-efficiency human osteosarcoma (MG63) cellular uptake of the NGO/ PEG/ICG/PTX. In turn, the in vivo results indicated a high accumulation of NGO/PEG/ICG/PTX in the MG63 tumor. The results also demonstrated that the tumor-bearing mice had complete tumor suppression without injury to healthy vital organs.

According to Usman et al., a theranostic system promising future chemotherapy for antineoplastic treatment with diagnostic modalities based on co-administration is proposed [268]. They synthesized a theranostic system based on GO composed of a natural chemotherapeutic agent, CA, whereas gadolinium (Gd) and gold (AuNPs) nanoparticles were used as contrast agents for the MRI. Subsequently, in vitro tests demonstrated that $90 \%$ of the chlorogenic acid was released at $\mathrm{pH} 4.8$, and HepG2 cancer cells had their growth inhibited with no significant effect on normal 3T3 cells. The MRI performed indicated an increase in contrast as compared with the simple Gd references. Additionally, Bansal et al. developed a theranostic tool for using GQDs with biosurfactants for cancer therapy. It has less toxicity, minimal side effects, and more biodegradability than other standards chemotherapy drugs used in cancer therapy [269]. This nanomaterial was conjugated with biosurfactant, and FA and the diagnostic and therapeutic effects against cancer cells with overexpression of folate receptors were studied. The cell viability test showed that GQDs were not toxic to cells. It was also observed that the conjugation of FA further increased the specificity of GQDs / bioconjugates concerning tumor cells. This result was strengthened by the laser scanning confocal microscopy results and the drug uptake test. It observed a more significant amount of drug uptake when GQDs / bioconjugates were decorated with folic acid.

On the other hand, Nasrollahi et al. developed nanoparticles of GQD conjugated with the scFvB10 antibody. Subsequently, cisplatin (CDDP) was loaded into GQDs/scFvB10 employing electrostatic interaction and complexation with carboxylic groups of GQDs [270]. The efficient mechanism for capturing and targeting GQDs/scFvB10 through epidermal growth factor receptor (EGFR)-mediated endocytosis was verified using the strong fluorescence of GQDs as bioimaging agents. It was found that GQDs/scFvB10 has a $\mathrm{pH}$ dependent release, higher for acid, and can be effectively absorbed by MDA-MB-231 breast cancer cells that overexpress EGFR. In addition, its high stability in physiological environments for several months, together with its negligible 
cytotoxicity, makes the nanostructure efficient for cellular images and as a carrier of CDDP.

\section{Toxicology}

GO has dose-dependent effects [271], shows mitochondrial damage, generates reactive oxygen species, and has pronounced antimicrobial properties [272-275]. GO and rGO affect cell proliferation, particularly osteogenic and cartilage types [276-279]. The effects on apoptosis of graphene oxide are controversial and dependent on cell type [271, 280]. Otherwise, graphene oxide nanoplatelets (GONP), graphene oxide nanoribbons (GONR), and graphene nano onions (GNO) are considered to be more toxic than GO and rGO. In addition, it has been shown that toxicity depends on the size and functional groups GONR was found to be more toxic than GONP [281] and more toxic than GOS [282]. These forms of graphene exhibit direct time- and dosedependent effects [282-284], causing DNA damage [282]. GONP, GONR, and GNO do not affect the mechanism of mesenchymal stem cell differentiation [283]. More significant cytotoxicity was observed due to increased adsorption of micronutrients on the hydrophobic surface of HHG sheets, limiting their availability [285]. Graphene quantum dots have almost no toxic properties up to a concentration of 200-320 $\mu \mathrm{g} / \mathrm{ml}[286,287]$. The GO affects cell proliferation, particularly osteogenic types [277], but has not shown toxic properties [288]. According to Salesa et al. [289] is essential to notice that toxicological differences between single-layer GO and multilayer GO may be observed and represent an important aspect.

One of the favorite models for toxicologists is the zebrafish. Many studies on the toxicity of graphene and its derivatives have been performed on this object [290-295]. In particular, it was found that graphene affects embryotoxicity, biochemistry, and behavior. The study with environmental concentrations (from 0.1 to $100 \mathrm{mg} / \mathrm{L}$ ) on multi-organ systems in developing zebrafish larvae was experimentally assessed. The pG and GO were found to accumulate in the brain tissue that also caused significant changes in the heartbeat and survival rate. The sizes of hepatocytes were reduced. In another study [291], authors used dosage from 10 to $100 \mathrm{mg} / \mathrm{L} \mathrm{GO}-\mathrm{COOH}$ specifically induced neurodevelopmental abnormalities and the altered tendency of locomotor in larval fish.

Furthermore, GO-COOH exposure led to increased AchE and ATPase activities and oxidative stress upregulation. Interestingly, it disrupted the expression of genes involved in neurodevelopment and neurotransmitter pathway, showing that Parkinson's disease-related genes' expression was disordered after $\mathrm{GO}-\mathrm{COOH}$ treatment. These results suggest that $\mathrm{GO}-\mathrm{COOH}$ can induce neurotoxicity and Parkinson's disease-like symptoms in zebrafish larvae.
Another case with s-GO has been shown to selectively inhibit glutamatergic transmission in neuronal cultures in vitro and brain explants obtained from animals injected with the nanomaterial. This raises the exciting prospect that s-GO can be developed as a platform for novel nervous system therapeutics [296]. Fascinating results were obtained by researchers [290] who studied the behavior of fish in the model of predator avoidance. An alteration in predator avoidance behavior was observed in all treated groups, while GR-treated fish exhibited abnormal exploratory behavior. The authors discovered that a high concentration of GR exposure significantly decreased several neurotransmitters and cortisol levels from the ELISA results.

There are relatively fewer works on the effect of graphene on insects. In many cases was shown that higher concentrations ( $>25 \mathrm{mg} / \mathrm{L}$ ), graphene, and derivates led to oxidative stress, ROS accumulation, and DNA damage and significantly reduced their survival rate [297-300]. Nowadays, graphene and its derivatives are seen as promising insecticides [301, 302].

\section{Conclusion}

Over the past few years, the potential applicability of nanomaterials of the graphene family has been gaining momentum due to their excellent optical, electronic, thermal, and mechanical properties.

In this direction, this review has shown the applicability of the graphene family in several biomedical modalities, with particular attention for cancer therapy and diagnosis, as a potent theranostic. This ability is derivative from the considerable number of forms that the graphene family can assume. It is essential to notice that the quality of the material produced governs biological behavior. Thus, the quality of production of these materials, especially for clinical application, must be appropriately addressed. Despite the growing increase in the bioavailability and toxicity studies of graphene and its derivatives, there is still much to be unveiled that would allow safe and effective formulations.

Acknowledgements The authors would like to thank CAPES (Finance Code 001, PROEX 23038.000509/2020-82), Funcap (PNE-011200048.01.00/16), FAPERJ (Rede NanoSaude), and Basal Program for Centers of Excellence, Conicyt Basal CEDENNA AFB180001, Fondecyt 1200782 for financial support rendered during this review.

\section{Declarations}

Conflict of interest The authors declare that there are no conflicts of interest. 


\section{References}

1. Park, W., Shin, H., Choi, B., Rhim, W.K., Na, K., Keun Han, D.: Advanced hybrid nanomaterials for biomedical applications. Prog. Mater. Sci. 114, 1-110 (2020)

2. Liu, Y., Ge, Z., Li, Z., Chen, Y.: High-power instant-synthesis technology of carbon nanomaterials and nanocomposites. Nano Energy 80, 1-61 (2021)

3. Zhang, M., Wang, W., Wu, F., Zheng, T., Ashley, J., Mohammadniaei, M., et al.: Biodegradable Poly( $\gamma$-glutamic acid)@ glucose oxidase@ carbon dot nanoparticles for simultaneous multimodal imaging and synergetic cancer therapy. Biomaterials 252, 120106 (2020)

4. Liu, Y., Lv, S., Liu, D., Song, F.: Recent development of amorphous metal coordination polymers for cancer therapy. Acta Biomater. 116, 16-31 (2020)

5. Yang, Y., Wang, S., Zhou, Y., Wang, X., Liu, X., Xie, A., et al.: Structurally accurate lipophilic Pt1 Ag28 nanoclusters based cancer theranostic micelles for dual-targeting/aggregation enhanced fluorescence imaging and photothermal/photodynamic therapies. Colloids Surf. B Biointerfaces. 196, 111346 (2020)

6. Shetty, A., Chandra, S.: Inorganic hybrid nanoparticles in cancer theranostics: understanding their combinations for better clinical translation. Mater. Today Chem. 18, 100381 (2020)

7. Khajeali, A., Khodadadi, R., Pirayesh Islamian, J.: Metal-based nanoparticles as radio-sensitizer in gastric cancer therapy. J. Drug Deliv. Sci. Technol. 56, 101576 (2020)

8. Wu, D., Shou, X., Zhang, Y., Li, Z., Wu, G., Wu, D., et al.: Cell membrane-encapsulated magnetic nanoparticles for enhancing natural killer cell-mediated cancer immunotherapy. Nanomedicine 32, 102333 (2021)

9. Gao, X., Li, L., Cai, X., Huang, Q., Xiao, J., Cheng, Y.: Targeting nanoparticles for diagnosis and therapy of bone tumors: Opportunities and challenges. Biomaterials 265, 120404 (2021)

10. Serrano-Aroca, Á., Takayama, K., Tuñón-Molina, A., Seyran, M., Hassan, S.S., Choudhury, P.P., et al.: Carbon-based nanomaterials: promising antiviral agents to combat COVID-19 in the microbial-resistant era. ACS Nano 15(5), 8069-8086 (2021)

11. Veclani, D., Tolazzi, M., Melchior, A.: Molecular interpretation of pharmaceuticals' adsorption on carbon nanomaterials: theory meets experiments. Process 8(6), 642 (2020)

12. Aliyev, E., Filiz, V., Khan, M.M., Lee, Y.J., Abetz, C., Abetz, V.: Structural characterization of graphene oxide: surface functional groups and fractionated oxidative debris. Nanomaterials 9(8), 1180 (2019)

13. Eckhart, K.E., Holt, B.D., Laurencin, M.G., Sydlik, S.A.: Covalent conjugation of bioactive peptides to graphene oxide for biomedical applications. Biomater. Sci. 7, 3876-3885 (2019)

14. Tabish, T., Pranjol, M., Horsell, D., Rahat, A., Whatmore, J., Winyard, P., et al.: Graphene oxide-based targeting of extracellular cathepsin $\mathrm{D}$ and cathepsin $\mathrm{L}$ as a novel anti-metastatic enzyme cancer therapy. Cancers (Basel). 11(3), 319 (2019)

15. Nakajima, T., Matsuo, Y.: Formation process and structure of graphite oxide. Carbon N Y. 32(3), 469-475 (1994)

16. Brodie, B.C.: XXIV-On the combination of carbonic oxide with potassium. Q. J. Chem. Soc. Lond. 12, 269-272 (1860)

17. Staudenmaier, L.: Verfahren zur Darstellung der Graphitsäure. Berichte der Dtsch. Chem. Gesellschaft. 32(2), 1394-1399 (1899)

18. Hummers, W.S., Offeman, R.E.: Preparation of graphitic oxide. J. Am. Chem. Soc. 80(6), 1339 (1958)

19. Yu, H., Zhang, B., Bulin, C., Li, R., Xing, R.: High-efficient synthesis of graphene oxide based on improved hummers method. Sci. Reports. 6(1), 36143 (2016)
20. Dimiev, A.M., Tour, J.M.: Mechanism of graphene oxide formation. ACS Nano 8(3), 3060-3068 (2014)

21. Abdelkader, A.M., Kinloch, I.A., Dryfe, R.A.W.: High-yield electro-oxidative preparation of graphene oxide. Chem. Commun. 50(61), 8402-8404 (2014)

22. Ženata, O., Panáček, A., Kvítek, L., Vrzal, R.: The impact of graphene oxide on androgen receptor signalling in prostate cancer cells. Chemosphere 269, 128759 (2021)

23. Wang, P., Wang, X., Tang, Q., Chen, H., Zhang, Q., Jiang, H., et al.: Functionalized graphene oxide against U251 glioma cells and its molecular mechanism. Mater. Sci. Eng. C. 116, 111187 (2020)

24. Liu, Q., Liu, J., Yang, H., Wang, X., Kong, J., Zhang, X.: Highly sensitive lung cancer DNA detection via GO enhancing eATRP signal amplification. Microchem. J. 160, 105766 (2021)

25. Gai, L.X., Wang, W.Q., Wu, X., Su, X.J., Yang, F.C.: NIR absorbing reduced graphene oxide for photothermal radiotherapy for treatment of esophageal cancer. J. Photochem. Photobiol. B Biol. 194, 188-193 (2019)

26. Slekiene, N., Snitka, V.: Impact of graphene oxide functionalized with doxorubicin on viability of mouse hepatoma MH-22A cells. Toxicol. In Vitro 65, 104821 (2020)

27. Qiu, Z., Hu, J., Li, Z., Yang, X., Hu, J., You, Q., et al.: Graphene oxide-based nanocomposite enabled highly efficient targeted synergistic therapy for colorectal cancer. Colloids Surf. A Physicochem. Eng. Asp. 593, 124585 (2020)

28. Thapa, R.K., Youn, Y.S., Jeong, J.H., Choi, H.G., Yong, C.S., Kim, J.O.: Graphene oxide-wrapped PEGylated liquid crystalline nanoparticles for effective chemo-photothermal therapy of metastatic prostate cancer cells. Colloids Surf. B Biointerfaces. 143, 271-277 (2016)

29. Fiorillo, M., Verre, A.F., Iliut, M., Peiris-Pagés, M., Ozsvari, B., Gandara, R., et al.: Graphene oxide selectively targets cancer stem cells, across multiple tumor types: Implications for nontoxic cancer treatment, via "differentiation-based nano-therapy." Oncotarget 6, 3553-3562 (2015)

30. Unal, M.A., Bayrakdar, F., Nazir, H., Besbinar, O., Gurcan, C., Lozano, N., et al.: Graphene oxide nanosheets interact and interfere with SARS-CoV-2 surface proteins and cell receptors to inhibit infectivity. Small 17(25), 2101483 (2021)

31. Hassanpour, S., Behnam, B., Baradaran, B., Hashemzaei, M., Oroojalian, F., Mokhtarzadeh, A., et al.: Carbon based nanomaterials for the detection of narrow therapeutic index pharmaceuticals. Talanta 221, 121610 (2021)

32. Priyadarsini, S., Mohanty, S., Mukherjee, S., Basu, S., Mishra, M.: Graphene and graphene oxide as nanomaterials for medicine and biology application. J. Nanostruct. Chem. 8, 123-137 (2018)

33. Alexander, A., Agrawal, M., Yadav, P., Jeswani, G., Verma, V.S., Siddique, S., et al.: Chapter 17 - Targeted delivery through carbon nanomaterials: applications in bioactive delivery systems. In: Singh, M.R., Singh, D., Kanwar, J.R., Chauhan, N.S. (eds.) Advances and avenues in the development of novel carriers for bioactives and biological agents, pp. 509-524. Academic Press, Massachusetts (2020)

34. Su, D., Li, H., Yan, X., Lin, Y., Lu, G.: Biosensors based on fluorescence carbon nanomaterials for detection of pesticides. Trends. Analyt. Chem. 134, 116126 (2021)

35. Salih, E., Mekawy, M., Hassan, R.Y.A., El-Sherbiny, I.M.: Synthesis, characterization and electrochemical-sensor applications of zinc oxide/graphene oxide nanocomposite. J. Nanostruct. Chem. 6, 137-144 (2016)

36. Khand, N.H., Palabiyik, I.M., Buledi, J.A., Ameen, S., Memon, A.F., Ghumro, T., et al.: Functional $\mathrm{Co} 3 \mathrm{O} 4$ nanostructure-based electrochemical sensor for direct determination of ascorbic acid in pharmaceutical samples. J. Nanostructure Chem. 11, 455-468 (2021) 
37. Aragaw, B.A.: Reduced graphene oxide-intercalated graphene oxide nano-hybrid for enhanced photoelectrochemical water reduction. J. Nanostructure Chem. 10, 9-18 (2019)

38. Rani, A., Reddy, R., Sharma, U., Mukherjee, P., Mishra, P., Kuila, A., et al.: A review on the progress of nanostructure materials for energy harnessing and environmental remediation. J. Nanostructure Chem. 8, 255-291 (2018)

39. Kumar, R., Aadil, K.R., Ranjan, S., Kumar, V.B.: Advances in nanotechnology and nanomaterials based strategies for neural tissue engineering. J. Drug Deliv. Sci. Technol. 57, 101617 (2020)

40. Rivera-Briso, A.L., Aachmann, F.L., Moreno-Manzano, V., Serrano-Aroca, Á.: Graphene oxide nanosheets versus carbon nanofibers: Enhancement of physical and biological properties of poly(3-hydroxybutyrate-co-3-hydroxyvalerate) films for biomedical applications. Int. J. Biol. Macromol. 143(15), 1000-1008 (2020)

41. Martí, M., Frígols, B., Salesa, B., Serrano-Aroca, Á.: Calcium alginate/graphene oxide films: reinforced composites able to prevent Staphylococcus aureus and methicillin-resistant Staphylococcus epidermidis infections with no cytotoxicity for human keratinocyte HaCaT cells. Eur. Polym. J. 110, 14-21 (2019)

42. Frígols, B., Martí, M., Salesa, B., Hernández-Oliver, C., Aarstad, O., Ulset, A.-S.T., et al.: Graphene oxide in zinc alginate films: antibacterial activity, cytotoxicity, zinc release, water sorption/ diffusion, wettability and opacity. PLoS ONE 14(3), 212819 (2019)

43. Salesa, B., Martí, M., Frígols, B., Serrano-Aroca, Á.: Carbon nanofibers in pure form and in calcium alginate composites films: new cost-effective antibacterial biomaterials against the life-threatening multidrug-resistant Staphylococcus epidermidis. Polymers 11(3), 453 (2019)

44. Elias, L., Taengua, R., Frígols, B., Salesa, B., Serrano-Aroca, Á.: Carbon nanomaterials and LED irradiation as antibacterial strategies against gram-positive multidrug-resistant pathogens. Int. J. Mol. Sci. 20(14), 3603 (2019)

45. Serrano-Aroca, Á., Ruiz-Pividal, J.-F., Llorens-Gámez, M.: Enhancement of water diffusion and compression performance of crosslinked alginate films with a minuscule amount of graphene oxide. Sci. Rep. 7, 1-8 (2017)

46. Serrano-Aroca, Á., Iskandar, L., Deb, S.: Green synthetic routes to alginate-graphene oxide composite hydrogels with enhanced physical properties for bioengineering applications. Eur. Polym. J. 103, 198-206 (2018)

47. Llorens-Gámez, M., Serrano-Aroca, Á.: Low-cost advanced hydrogels of calcium alginate/carbon nanofibers with enhanced water diffusion and compression properties. Polymers 10(4), 405 (2018)

48. Sánchez-Correa, F., Vidaurre-Agut, C., Serrano-Aroca, Á., Campillo-Fernández, A.J.: Poly(2-hydroxyethyl acrylate) hydrogels reinforced with graphene oxide: Remarkable improvement of water diffusion and mechanical properties. J. Appl. Polym. Sci. 135, 46158 (2018)

49. Llorens-Gámez, M., Salesa, B., Serrano-Aroca, Á.: Physical and biological properties of alginate/carbon nanofibers hydrogel films. Int. J. Biol. Macromol. 151, 499-507 (2020)

50. Salesa, B., Llorens-Gámez, M., Serrano-Aroca, Á.: Study of 1D and $2 \mathrm{D}$ carbon nanomaterial in alginate films. Nanomaterials 10(2), 206 (2020)

51. Serra, R.S., Molina-Mateo, J., Torregrosa-Cabanilles, C., Andrio-Balado, A., Dueñas, J.M.M., Serrano-Aroca, Á.: Bionanocomposite hydrogel based on zinc alginate/graphene oxide: morphology, structural conformation, thermal behavior/degradation, and dielectric properties. Polymers 12(3), 702 (2020)

52. Grasseschi, D., Silva, W.C., Souza Paiva, R., Starke, L.D., Nascimento, A.S.: Surface coordination chemistry of graphene:
Understanding the coordination of single transition metal atoms. Coord. Chem. Rev. 422, 213469 (2020)

53. Homaeigohar, S., Elbahri, M.: Graphene membranes for water desalination. NPG Asia Mater. 9, 427 (2017)

54. Zhou, F., Fathizadeh, M., Yu, M.: Single- to few-layered, graphene-based separation membranes. Annu. Rev. Chem. Biomol. Eng. 9, 17-39 (2018)

55. Georgakilas, V., Tiwari, J.N., Kemp, K.C., Perman, J.A., Bourlinos, A.B., Kim, K.S., et al.: Noncovalent functionalization of graphene and graphene oxide for energy materials, biosensing, catalytic, and biomedical applications. Chem. Rev. 116(9), 54645519 (2016)

56. Bhuyan, M.S.A., Uddin, M.N., Islam, M.M., Bipasha, F.A., Hossain, S.S.: Synthesis of graphene. Int. Nano. Lett. 6, 65-83 (2016)

57. Torres, T.: Graphene chemistry. Chem. Soc. Rev. 46, 4385-4386 (2017)

58. Moaddeli, M., Salehi, H., Amiri, P.: Physisorption mechanism in graphene/noble metal (111)/Ni(111) heterostructures: an abinitio study. J. Appl. Phys. 120, 75301 (2016)

59. Dong, J.C., Li, H., Sun, F.W., Li, Y.F.: Importance of coupling pattern and chemical decoration for graphene nanotransistors. J. Phys. Chem. C. 116(11), 6762-6769 (2012)

60. Arvizu-Rodriguez, L.E., Paramo-García, U., Caballero-Briones, F.: Low resistance, high mobility reduced graphene oxide films prepared with commercial antioxidant supplements. Mater. Lett. 276, 128176 (2020)

61. Palanisamy, S., Thirumalraj, B., Chen, S.-M., Wang, Y.-T., Velusamy, V., Ramaraj, S.K.: A facile electrochemical preparation of reduced graphene oxide@ polydopamine composite: a novel electrochemical sensing platform for amperometric detection of chlorpromazine. Sci. Rep. 6, 1-9 (2016)

62. Kavitha, C.: A review on reduced Graphene oxide hybrid nano composites and their prominent applications. Mater. Today Proc. (2021). https://doi.org/10.1016/J.MATPR.2021.05.343

63. Rout, D.R., Jena, H.M.: Removal of malachite green dye from aqueous solution using reduced graphene oxide as an adsorbent. Mater. Today Proc. (2021). https://doi.org/10.1016/J.MATPR. 2021.03.406

64. Ansari, M.Z., Siddiqui, W.A.: Deoxygenation of graphene oxide using biocompatible reducing agent Ficus carica (dried ripe fig). J. Nanostructure Chem. 8, 431-440 (2018)

65. Guex, L.G., Sacchi, B., Peuvot, K.F., Andersson, R.L., Pourrahimi, A.M., Ström, V., et al.: Experimental review: chemical reduction of graphene oxide (GO) to reduced graphene oxide (rGO) by aqueous chemistry. Nanoscale 9(27), 9562-9571 (2017)

66. Lavin-Lopez, M.P., Paton-Carrero, A., Sanchez-Silva, L., Valverde, J.L., Romero, A.: Influence of the reduction strategy in the synthesis of reduced graphene oxide. Adv. Powder Technol. 28(12), 3195-3203 (2017)

67. Yoon, Y., Kye, H., Yang, W.S., Kang, J.-W.: Comparing graphene oxide and reduced graphene oxide as blending materials for polysulfone and polyvinylidene difluoride membranes. Appl. Sci. 10(6), 1-10 (2020)

68. Nimbalkar, A.S., Tiwari, S.K., Ha, S.K., Hong, C.K.: An efficient water saving step during the production of graphene oxide via chemical exfoliation of graphite. Mater. Today Proc. 21, 17491754 (2020)

69. Balaji, A., Yang, S., Wang, J., Zhang, J.: Graphene Oxide-Based Nanostructured DNA Sensor. Biosensors 9(2), 74 (2019)

70. Halim, A., Luo, Q., Ju, Y., Song, G.: A mini review focused on the recent applications of graphene oxide in stem cell growth and differentiation. Nanomater. 8(9), 736 (2018)

71. Lin, L., Peng, H., Liu, Z.: Synthesis challenges for graphene industry. Nat. Mater. 18, 520-524 (2019) 
72. Huang, S., Panes-Ruiz, L.A., Croy, A., Löffler, M., Khavrus, V., Bezugly, V., et al.: Highly sensitive room temperature ammonia gas sensor using pristine graphene: The role of biocompatible stabilizer. Carbon N Y. 173, 262-270 (2021)

73. Taniselass, S., Arshad, M.K., Gopinath, S.C.B.: Current state of green reduction strategies: solution-processed reduced graphene oxide for healthcare biodetection. Mater. Sci. Eng. C. 96, 904-914 (2019)

74. Ryan, A.J., Kearney, C.J., Shen, N., Khan, U., Kelly, A.G., Probst, C., et al.: Electroconductive biohybrid collagen/pristine graphene composite biomaterials with enhanced biological activity. Adv. Mater. 30(15), 1706442 (2018)

75. Lin, T.-N., Santiago, S.R.M., Yuan, C.-T., Chiu, K.-P., Shen, J.-L., Wang, T.-C., et al.: Enhanced performance of GaN-based ultraviolet light emitting diodes by photon recycling using graphene quantum dots. Sci Rep. 7, 1-9 (2017)

76. Fasbender, S., Zimmermann, L., Cadeddu, R.P., Luysberg, M., Moll, B., Janiak, C., Heinzel, , et al.: The low toxicity of graphene quantum dots is reflected by marginal gene expression changes of primary human hematopoietic stem cells. Sci. Rep. 9, 1-13 (2019)

77. Iravani, S., Varma, R.S.: Green synthesis, biomedical and biotechnological applications of carbon and graphene quantum dots. A review. Environ. Chem. Lett. 18, 703-727 (2020)

78. Tian, P., Tang, L., Teng, K.S., Lau, S.P.: Graphene quantum dots from chemistry to applications. Mater. Today Chem. 10, 221-258 (2018)

79. Tran, H.L., Darmanto, W., Doong, R.A.: Electrochemical immunosensor for ultra-sensitive detection of attomolar prostate specific antigen with sulfur-doped graphene quantum dot@gold nanostar as the probe. Electrochim. Acta 389, 138700 (2021)

80. Xin, Q., Shah, H., Xie, W., Wang, Y., Jia, X., Nawaz, A., et al.: Preparation of blue- and green-emissive nitrogen-doped graphene quantum dots from graphite and their application in bioimaging. Mater. Sci. Eng C. 119, 111642 (2021)

81. Campbell, E., Hasan, M.T., Gonzalez-Rodriguez, R., Truly, T., Lee, B.H., Green, K.N., et al.: Graphene quantum dot formulation for cancer imaging and redox-based drug delivery. Nanomedicine 38, 102408 (2021)

82. Yu, Z., Ma, W., Wu, T., Wen, J., Zhang, Y., Wang, L., et al.: Coumarin-modified graphene quantum dots as a sensing platform for multicomponent detection and its applications in fruits and living cells. ACS Omega 5(13), 7369-7378 (2020)

83. Orsu, P., Koyyada, A.: Recent progresses and challenges in graphene based nano materials for advanced therapeutical applications: a comprehensive review. Mater. Today Commun. 22, 100823 (2020)

84. Serrano-Aroca, Á., Deb, S.: Synthesis of irregular graphene oxide tubes using green chemistry and their potential use as reinforcement materials for biomedical applications. PLoS ONE 12(9), 185235 (2017)

85. Vieira Segundo, J.E.D., Vilar, E.O.: Grafeno: Uma revisão sobre propriedades, mecanismos de produção e potenciais aplicações em sistemas energéticos. REMAP 11(12), 54-57 (2016)

86. Zhang, T., Xue, Q., Zhang, S., Dong, M.: Theoretical approaches to graphene and graphene-based materials. Nano Today 7(3), 180-200 (2012)

87. Morozov, S.V., Novoselov, K.S., Katsnelson, M.I., Schedin, F., Elias, D.C., Jaszczak, J.A., et al.: giant intrinsic carrier mobilities in graphene and its bilayer. Phys. Rev. Lett. 100(1), 1-11 (2008)

88. Lee, J.-U., Yoon, D., Cheong, H.: Estimation of Young's Modulus of Graphene by Raman Spectroscopy. Nano Lett. 12(9), 4444-4448 (2012)

89. Fu, Y., Hansson, J., Liu, Y., Chen, S., Zehri, A., Samani, M.K., et al.: Graphene related materials for thermal management. 2D Mater. 7(1), 12001 (2019)
90. Dahanayaka, M., Liu, B., Srikanth, N., Zhou, K.: Ionised graphene oxide membranes for seawater desalination. Desalination 496, 114637 (2020)

91. Dash, P., Rout, T.K., Biswal, S.K.: Study on the preparation of GO and RGO by chemical and mechanical exfoliation of natural graphite for the aluminum industry. J. Sustain. Metall. 6, 26-33 (2019)

92. Price, R.J., Ladislaus, P.I., Smith, G.C., Davies, T.J.: A novel 'bottom-up' synthesis of few- and multi-layer graphene platelets with partial oxidation via cavitation. Ultrason. Sonochem. 56, 466-473 (2019)

93. Tour, J.M.: Top-down versus bottom-up fabrication of graphenebased electronics. Chem. Mater. 26(1), 163-171 (2013)

94. Reina, G., González-Domínguez, J.M., Criado, A., Vázquez, E., Bianco, A., Prato, M.: Promises, facts and challenges for graphene in biomedical applications. Chem. Soc. Rev. 46(15), 4400-4416 (2017)

95. Sturala, J., Luxa, J., Pumera, M., Sofer, Z.: Chemistry of graphene derivatives: synthesis, applications, and perspectives. Chemistry 24(23), 5992-6006 (2018)

96. Bhuyan, M.S.A., Uddin, M.N., Islam, M.M., Bipasha, F.A., Hossain, S.S.: Synthesis of graphene. Int. Nano Lett. 6, 65-83 (2016)

97. Lee, X.J., Hiew, B.Y.Z., Lai, K.C., Lee, L.Y., Gan, S., Thangalazhy-Gopakumar, S., et al.: Review on graphene and its derivatives: synthesis methods and potential industrial implementation. J. Taiwan Inst. Chem. Eng. 98, 163-180 (2019)

98. Yan, Y., Nashath, F.Z., Chen, S., Manickam, S., Lim, S.S., Zhao, H., et al.: Synthesis of graphene: potential carbon precursors and approaches. Nanotechnol. Rev. 9(1), 1284-1314 (2020)

99. Guerrero-Contreras, J., Caballero-Briones, F.: Graphene oxide powders with different oxidation degree, prepared by synthesis variations of the Hummers method. Mater. Chem. Phys. 153, 209-220 (2015)

100. Patil, R., Patel, H., Pillai, S.B., Jha, P.K., Bahadur, P., Tiwari, S.: Influence of surface oxygen clusters upon molecular stacking of paclitaxel over graphene oxide sheets. Mater. Sci. Eng. C. 116, $111232(2020)$

101. Hofmann, U., Holst, R.: Über die Säurenatur und die Methylierung von Graphitoxyd. Berichte der Dtsch. Chem. Gesellschaft. 72(4), 754-771 (1939)

102. Ruess, G., Vogt, F.: Höchstlamellarer Kohlenstoff aus Graphitoxyhydroxyd. Monatsh. Chem. 78, 222-242 (1948)

103. Scholz, W., Boehm, H.P.: Untersuchungen am GraphitoxidVI Betrachtungen zur Struktur des Graphitoxids. Z. Anorg. Allg Chem. 369(36), 327-340 (1969)

104. Lerf, A., He, H., Forster, M., Klinowski, J.: Structure of graphite oxide revisited. J. Phys. Chem. B. 102(23), 4477-4482 (1998)

105. Savazzi, F., Risplendi, F., Mallia, G., Harrison, N.M., Cicero, G.: Unravelling some of the structure-property relationships in graphene oxide at low degree of oxidation. J. Phys. Chem. Lett. 9(7), 1746-1749 (2018)

106. Szabó, T., Berkesi, O., Forgó, P., Josepovits, K., Sanakis, Y., Petridis, D., et al.: Evolution of surface functional groups in a series of progressively oxidized graphite oxides. Chem. Mater. 18(11), 2740-2749 (2006)

107. Cai, W., Piner, R.D., Stadermann, F.J., Park, S., Shaibat, M.A., Ishii, Y., et al.: Synthesis and solid-state NMR structural characterization of 13C-labeled graphite oxide. Science 321(5897), 1815-1817 (2008)

108. Casabianca, L.B., Shaibat, M.A., Cai, W.W., Park, S., Piner, R., Ruoff, R.S., et al.: NMR-based structural modeling of graphite oxide using multidimensional 13C solid-state NMR and ab initio chemical shift calculations. J. Am. Chem. Soc. 132(16), 56725676 (2010)

109. Bagri, A., Mattevi, C., Acik, M., Chabal, Y.J., Chhowalla, M., Shenoy, V.B.: Structural evolution during the reduction 
of chemically derived graphene oxide. Nat. Chem. 2, 581-587 (2010)

110. Gandhiraman, R.P., Nordlund, D., Javier, C., Koehne, J.E., Chen, B., Meyyappan, M.: X-ray absorption study of graphene oxide and transition metal oxide nanocomposites. J. Phys. Chem. C. 118(32), 18706-18712 (2014)

111. Ganguly, A., Sharma, S., Papakonstantinou, P., Hamilton, J.: Probing the thermal deoxygenation of graphene oxide using high-resolution in situ X-ray-based spectroscopies. J. Phys. Chem. C. 115(34), 17009-17019 (2011)

112. Lee, D.W., De Los Santos, L.V., Seo, J.W., Felix, L.L., Bustamante, D.A., Cole, J.M., et al.: The structure of graphite oxide: investigation of its surface chemical groups. J. Phys. Chem. B. 114(17), 5723-5728 (2010)

113. Kudin, K.N., Ozbas, B., Schniepp, H.C., Prudhomme, R.K., Aksay, I.A., Car, R.: Raman spectra of graphite oxide and functionalized graphene sheets. Nano Lett. 8(1), 36-41 (2007)

114. Gao, W., Alemany, L.B., Ci, L., Ajayan, P.M.: New insights into the structure and reduction of graphite oxide. Nat. Chem. 1, 403-408 (2009)

115. Erickson, K., Erni, R., Lee, Z., Alem, N., Gannett, W., Zettl, A.: Determination of the local chemical structure of graphene oxide and reduced graphene oxide. Adv. Mater. 22(40), 4467-4472 (2010)

116. Fujii, S., Enoki, T.: Clar's aromatic sextet and $\pi$-electron distribution in nanographene. Angew. Chemie Int. Ed. 51(29), 7236-7241 (2012)

117. Mkhoyan, K.A., Contryman, A.W., Silcox, J., Stewart, D.A., Eda, G., Mattevi, C., et al.: Atomic and electronic structure of graphene-oxide. Nano Lett. 9(3), 1058-1063 (2009)

118. Kanakia, S., Toussaint, J.D., Chowdhury, S.M., Tembulkar, T., Lee, S., Jiang, Y.P., et al.: Dose ranging, expanded acute toxicity and safety pharmacology studies for intravenously administered functionalized graphene nanoparticle formulations. Biomaterials 35(25), 7022-7031 (2014)

119. Chen, J., Yao, B., Li, C., Shi, G.: An improved Hummers method for eco-friendly synthesis of graphene oxide. Carbon N Y. 64, 225-229 (2013)

120. Eigler, S.: Graphite sulphate-a precursor to graphene. Chem. Commun. 51(15), 3162-3165 (2015)

121. Marcano, D.C., Kosynkin, D.V., Berlin, J.M., Sinitskii, A., Sun, Z., Slesarev, A., et al.: Improved synthesis of graphene oxide. ACS Nano 4(8), 4806-4814 (2010)

122. Chen, J., Zhang, Y., Zhang, M., Yao, B., Li, Y., Huang, L., et al.: Water-enhanced oxidation of graphite to graphene oxide with controlled species of oxygenated groups. Chem. Sci. 7(3), 18741881 (2016)

123. Gao, W.: Synthesis, structure, and characterizations. In: Gao, W. (ed.) Graphene oxide, pp. 1-28. Springer, Cham (2015)

124. Kumar, N., Sinha Ray, S.: Synthesis and functionalization of nanomaterials. In: Sinha Ray, S. (ed.) Processing of polymerbased nanocomposites, pp. 15-55. Springer, Cham (2018)

125. Popescu, R.C., Andronescu, E., Vasile, B.S.: Recent advances in magnetite nanoparticle functionalization for nanomedicine. Nanomaterials 9(12), 1791 (2019)

126. Mirshafiee, V., Kim, R., Park, S., Mahmoudi, M., Kraft, M.L.: Impact of protein pre-coating on the protein corona composition and nanoparticle cellular uptake. Biomaterials 75, 295-304 (2016)

127. Russell, L.M., Liu, C.H., Grodzinski, P.: Nanomaterials innovation as an enabler for effective cancer interventions. Biomaterials 242, 119926 (2020)

128. Patel, P., et al.: Surface modification of nanoparticles for targeted drug delivery. In: Pathak, Y. (ed.) Surface modification of nanoparticles for targeted drug delivery, pp. 19-31. Springer, Cham (2019)
129. Sun, X., Cai, W., Chen, X.: Positron emission tomography imaging using radiolabeled inorganic nanomaterials. Acc. Chem. Res. 48(2), 286-294 (2015)

130. Lonkar, S.P., Deshmukh, Y.S., Abdala, A.A.: Recent advances in chemical modifications of graphene. Nano Res. 8, 10391074 (2015)

131. Yan, F., Jiang, Y., Sun, X., Bai, Z., Zhang, Y., Zhou, X.: Surface modification and chemical functionalization of carbon dots: a review. Microchim. Acta. 185, 1-34 (2018)

132. Begum, H., Ahmed, M.S., Jeon, S.: New approach for porous chitosan-graphene matrix preparation through enhanced amidation for synergic detection of dopamine and uric acid. ACS Omega 2(6), 3043-3054 (2017)

133. Niu, X., Yang, X., Mo, Z., Guo, R., Liu, N., Zhao, P., et al.: Perylene-functionalized graphene sheets modified with $\beta$-cyclodextrin for the voltammetric discrimination of phenylalanine enantiomers. Bioelectrochemistry 129, 189-198 (2019)

134. Ye, Q., Guo, L., Wu, D., Yang, B., Tao, Y., Deng, L., et al.: Covalent functionalization of bovine serum albumin with graphene quantum dots for stereospecific molecular recognition. Anal. Chem. 91(18), 11864-11871 (2019)

135. Li, Y., Wang, Q., Zhang, Y., Deng, D., He, H., Luo, L., et al.: A label-free electrochemical aptasensor based on graphene oxide/double-stranded DNA nanocomposite. Colloids Surf. B Biointerfaces. 145, 160-166 (2016)

136. Kosynkin, D.V., Higginbotham, A.L., Sinitskii, A., Lomeda, J.R., Dimiev, A., Price, B.K., et al.: Longitudinal unzipping of carbon nanotubes to form graphene nanoribbons. Nature $\mathbf{4 5 8}$, 872-876 (2009)

137. Niyogi, S., Bekyarova, E., Itkis, M.E., Zhang, H., Shepperd, K., Hicks, J., et al.: Spectroscopy of covalently functionalized graphene. Nano Lett. 10(10), 4061-4066 (2010)

138. Imani, R., Emami, S.H., Faghihi, S.: Synthesis and characterization of an octaarginine functionalized graphene oxide nanocarrier for gene delivery applications. Phys. Chem. Chem. Phys. 17(9), 6328-6339 (2015)

139. Abdollahi, Z., Taheri-Kafrani, A., Bahrani, S.A., Kajani, A.A.: PEGAylated graphene oxide/superparamagnetic nanocomposite as a high-efficiency loading nanocarrier for controlled delivery of methotrexate. J. Biotechnol. 298, 88-97 (2019)

140. Liu, T., Li, J., Wu, X., Zhang, S., Lu, Z., Li, G., et al.: Transferrin-targeting redox hyperbranched poly(amido amine)functionalized graphene oxide for sensitized chemotherapy combined with gene therapy to nasopharyngeal carcinoma. Drug Deliv. 26(1), 744-755 (2019)

141. Yang, L., Wang, F., Han, H., Yang, L., Zhang, G., Fan, Z.: Functionalized graphene oxide as a drug carrier for loading pirfenidone in treatment of subarachnoid hemorrhage. Colloids Surf. B Biointerfaces. 129, 21-29 (2015)

142. Sun, L., Fugetsu, B.: Mass production of graphene oxide from expanded graphite. Mater. Lett. 109, 207-210 (2013)

143. Wang, D., Zhang, G., Zhou, L., Wang, M., Cai, D., Wu, Z.: Synthesis of a multifunctional graphene oxide-based magnetic nanocomposite for efficient removal of $\mathrm{Cr}(\mathrm{VI})$. Langmuir 33(28), 7007-7014 (2017)

144. Xiao, F., Guo, M., Wang, J., Yan, X., Li, H., Qian, C., et al.: Ferrocene-terminated dendrimer functionalized graphene oxide layered sensor toward highly sensitive evaluation of Di(2-ethylhexyl) phthalate in liquor samples. Anal. Chim. Acta. 1043, 35-44 (2018)

145. Li, G., Zhao, H., Hong, J., Quan, K., Yuan, Q., Wang, X.: Antifungal graphene oxide-borneol composite. Colloids Surf. B Biointerfaces. 160, 220-227 (2017)

146. Meng, N., Su, Y.T., Zhou, N., Zhang, M., Shao, M., Fan, Y., et al.: Carboxylated graphene oxide functionalized with 
$\beta$-cyclodextrin-engineering of a novel nanohybrid drug carrier. Int. J. Biol. Macromol. 93, 117-122 (2016)

147. Sarvari, R., Sattari, S., Massoumi, B., Agbolaghi, S., BeygiKhosrowshahi, Y., Kahaie-Khosrowshahi, A.: Composite electrospun nanofibers of reduced graphene oxide grafted with poly(3dodecylthiophene) and poly(3-thiophene ethanol) and blended with polycaprolactone. J. Biomater. Sci. Polym. Ed. 28(15), 1740-1761 (2017)

148. Wu, D., Bäckström, E., Hakkarainen, M.: Starch derived nanosized graphene oxide functionalized bioactive porous starch scaffolds. Macromol. Biosci. 17(6), 1600397 (2017)

149. Raheem, A.A., Mahroos, A., Mahmoud, M.S., Ashour, I.: Fabrication of conductive human bio-nanoelectrode from graphene oxide modified with polyvinyl alcohol. IET Nanobiotechnol. 13(1), 1-5 (2019)

150. Velasco-Santos, C., Martínez-Hernández, A.L., Lozada-Cassou, M., Alvarez-Castillo, A., Castaño, V.M.: Chemical functionalization of carbon nanotubesthrough an organosilane. Nanotechnology 13(4), 495 (2002)

151. Wen, T., Yang, B., Guo, Y., Sun, J., Zhao, C., Zhang, S., et al.: Organosilane-functionalized graphene quantum dots and their encapsulation into bi-layer hollow silica spheres for bioimaging applications. Phys. Chem. Chem. Phys. 16(42), 23188-23195 (2014)

152. Hassan, A.M., Wan Ibrahim, W.A., Bakar, M.B., Sanagi, M.M., Sutirman, Z.A., Nodeh, H.R., et al.: New effective 3-aminopropyltrimethoxysilane functionalized magnetic sporopolleninbased silica coated graphene oxide adsorbent for removal of $\mathrm{Pb}$ (II) from aqueous environment. J. Environ. Manag. 253, $109658(2020)$

153. Song, S., Wang, Y., Shen, H., Zhang, J., Mo, H., Xie, J., et al.: Ultrasmall graphene oxide modified with $\mathrm{Fe} 3 \mathrm{O} 4$ nanoparticles as a fenton-like agent for methylene blue degradation. ACS Appl. Nano Mater. 2(11), 7074-7084 (2019)

154. Song, S., Ma, Y., Shen, H., Zhang, M., Zhang, Z.: Removal and recycling of ppm levels of methylene blue from an aqueous solution with graphene oxide. RSC Adv. 5(35), 27922-27932 (2015)

155. Yu, Z., Chen, Q., Lv, L., Pan, Y., Zeng, G., He, Y.: Attached $\beta$-cyclodextrin/ $\gamma$-(2,3-epoxypropoxy) propyl trimethoxysilane to graphene oxide and its application in copper removal. Water Sci. Technol. 75(10), 2403-2411 (2017)

156. Song, S., Wan, M., Feng, W., Zhang, J., Mo, H., Jiang, X., et al.: Graphene oxide as the potential vector of hydrophobic pesticides: ultrahigh pesticide loading capacity and improved antipest activity. ACS Agric. Sci. Technol. 1(3), 182-191 (2021)

157. Song, S., Wang, Y., Xie, J., Sun, B., Zhou, N., Shen, H., et al.: Carboxymethyl chitosan modified carbon nanoparticle for controlled emamectin benzoate delivery: improved solubility, $\mathrm{pH}$ responsive release, and sustainable pest control. ACS Appl. Mater. Interfaces. 11(37), 34258-34267 (2019)

158. Mahmoud, M.E., Amira, M.F., Seleim, S.M., Nabil, G.M., Abouelanwar, M.E.: Multifunctionalized graphene oxide@ nanopolyaniline@zirconium silicate nanocomposite for rapid microwable removal of dyes. J. Nanostruct. Chem. (2021). https://doi. org/10.1007/s40097-021-00390-0

159. Malinga, N.N., Jarvis, A.L.L.: Synthesis, characterization and magnetic properties of $\mathrm{Ni}, \mathrm{Co}$ and $\mathrm{FeCo}$ nanoparticles on reduced graphene oxide for removal of $\mathrm{Cr}(\mathrm{VI})$. J. Nanostruct. Chem. 10, 55-68 (2020)

160. de Menezes, F.D., Alencar, L.M.R., dos Santos, C.C., da Silva, M.I.B., Santos-Oliveira, R.: Using graphene quantum dots for treating radioactive liquid waste. Environ. Sci. Pollut. Res. 27, 3508-3512 (2019)

161. Patil, R., Bahadur, P., Tiwari, S.: Dispersed graphene materials of biomedical interest and their toxicological consequences. Adv. Colloid Interface Sci. 275, 102051 (2020)
162. Wu, Y., Okesola, B.O., Xu, J., Korotkin, I., Berardo, A., Corridori, I., et al.: Disordered protein-graphene oxide co-assembly and supramolecular biofabrication of functional fluidic devices. Nat. Commun. 11, 1-12 (2020)

163. Cao, M., Wang, N., Wang, L., Zhang, Y., Chen, Y., Xie, Z., et al.: Direct exfoliation of graphite into graphene in aqueous solutions of amphiphilic peptides. J. Mater. Chem. B. 4(1), 152-161 (2015)

164. Saravanan, N., Balamurugan, M., Devi, K.S.S., Nam, K.T., Kumar, A.S.: Vitamin B12-immobilized graphene oxide for efficient electrocatalytic carbon dioxide reduction reaction. Chemsuschem 13(21), 5620-5624 (2020)

165. Ismail, N.A., Zulkifli, N.W.M., Chowdhury, Z.Z., Johan, M.R.: Functionalization of graphene-based materials: effective approach for enhancement of tribological performance as lubricant additives. Diam. Relat. Mater. 115, 108357 (2021)

166. Baek, S., Kim, J., Kim, H., Park, S., Ban, H.W., Gu, D.H., et al.: Controlled grafting of colloidal nanoparticles on graphene through tailored electrostatic interaction. ACS Appl. Mater. Interfaces. 11(12), 11824-11833 (2019)

167. Matthews, R.P., Welton, T., Hunt, P.A.: Competitive pi interactions and hydrogen bonding within imidazolium ionic liquids. Phys. Chem. Chem. Phys. 16(7), 3238-3253 (2014)

168. Chauhan, N., Maekawa, T., Kumar, D.N.S.: Graphene based biosensors-accelerating medical diagnostics to new-dimensions. J. Mater. Res. 32, 2860-2882 (2017)

169. Oz, Y., Barras, A., Sanyal, R., Boukherroub, R., Szunerits, S., Sanyal, A.: Functionalization of reduced graphene oxide via thiol-maleimide "Click" chemistry: facile fabrication of targeted drug delivery vehicles. ACS Appl. Mater. Interfaces. 9(39), 34194-34203 (2017)

170. Díez-Pascual, A.M., Díez-Vicente, A.L.: Poly(propylene fumarate)/polyethylene glycol-modified graphene oxide nanocomposites for tissue engineering. ACS Appl. Mater. Interfaces. 8(28), 17902-17914 (2016)

171. Plachá, D., Jampilek, J.: Graphenic materials for biomedical applications. Nanomaterials 9(12), 1758 (2019)

172. Yu, W., Sisi, L., Haiyan, Y., Jie, L.: Progress in the functional modification of graphene/graphene oxide: a review. RSC Adv. 10(26), 15328-15345 (2020)

173. Jin, Z., McNicholas, T.P., Shih, C.-J., Wang, Q.H., Paulus, G.L.C., Hilmer, A.J., et al.: Click chemistry on solution-dispersed graphene and monolayer CVD graphene. Chem. Mater. 23(14), 3362-3370 (2011)

174. Georgakilas, V., Otyepka, M., Bourlinos, A.B., Chandra, V., Kim, N., Kemp, K.C., et al.: Functionalization of graphene: covalent and non-covalent approaches, derivatives and applications. Chem. Rev. 112(11), 6156-6214 (2012)

175. Sinitskii, A., Dimiev, A., Corley, D.A., Fursina, A.A., Kosynkin, D.V., Tour, J.M.: Kinetics of diazonium functionalization of chemically converted graphene nanoribbons. ACS Nano 4(4), 1949-1954 (2010)

176. Pieklarz, K., Tylman, M., Modrzejewska, Z.: Applications of chitosan-graphene oxide nanocomposites in medical science: a review. Prog. Chem. Appl. Chitin. Deriv. 23, 5-24 (2018)

177. Mahdavi, M., Rahmani, F., Nouranian, S.: Molecular simulation of $\mathrm{pH}$-dependent diffusion, loading, and release of doxorubicin in graphene and graphene oxide drug delivery systems. J. Mater. Chem. B. 4(46), 7441-7451 (2016)

178. Wei, Y., Zhou, F., Zhang, D., Chen, Q., Xing, D.: A graphene oxide based smart drug delivery system for tumor mitochondriatargeting photodynamic therapy. Nanoscale 8(6), 3530-3538 (2016)

179. Li, J., Lyv, Z., Li, Y., Liu, H., Wang, J., Zhan, W., et al.: A theranostic prodrug delivery system based on Pt(IV) conjugated nanographene oxide with synergistic effect to enhance the therapeutic efficacy of Pt drug. Biomaterials 51, 12-21 (2015) 
180. Tang, Y., Hu, H., Zhang, M.G., Song, J., Nie, L., Wang, S., et al.: An aptamer-targeting photoresponsive drug delivery system using "off-on" graphene oxide wrapped mesoporous silica nanoparticles. Nanoscale 7(14), 6304-6310 (2015)

181. Luo, H., Ao, H., Li, G., Li, W., Xiong, G., Zhu, Y., et al.: Bacterial cellulose/graphene oxide nanocomposite as a novel drug delivery system. Curr. Appl. Phys. 17(2), 249-254 (2017)

182. Masoudipour, E., Kashanian, S., Maleki, N.: A targeted drug delivery system based on dopamine functionalized nano graphene oxide. Chem. Phys. Lett. 668(16), 56-63 (2017)

183. Rao, Z., Ge, H., Liu, L., Zhu, C., Min, L., Liu, M., et al.: Carboxymethyl cellulose modified graphene oxide as $\mathrm{pH}$-sensitive drug delivery system. Int. J. Biol. Macromol. 107, 1184-1192 (2018)

184. Pan, Q., Lv, Y., Williams, G.R., Tao, L., Yang, H., Li, H., et al.: Lactobionic acid and carboxymethyl chitosan functionalized graphene oxide nanocomposites as targeted anticancer drug delivery systems. Carbohydr. Polym. 151, 812-820 (2016)

185. Yousefi, M., Dadashpour, M., Hejazi, M., Hasanzadeh, M., Behnam, B., de la Guardia, M., et al.: Anti-bacterial activity of graphene oxide as a new weapon nanomaterial to combat multidrug-resistance bacteria. Mater. Sci. Eng. C. 74, 568-581 (2017)

186. Pi, J., Shen, L., Shen, H., Yang, E., Wang, W., Wang, R., et al.: Mannosylated graphene oxide as macrophage-targeted delivery system for enhanced intracellular M. tuberculosis killing efficiency. Mater. Sci. Eng. C. 103, 109777 (2019)

187. Fan, L., Ge, H., Zou, S., Xiao, Y., Wen, H., Li, Y., et al.: Sodium alginate conjugated graphene oxide as a new carrier for drug delivery system. Int. J. Biol. Macromol. 93, 582-590 (2016)

188. Hashemi, H., Namazi, H.: Sonochemically synthesized blue fluorescent functionalized graphene oxide as a drug delivery system. Ultrason. Sonochem. 42, 124-133 (2018)

189. Wang, L., Hu, C., Shao, L.: The antimicrobial activity of nanoparticles: present situation and prospects for the future. Int. J. Nanomed. 12, 1227-1249 (2017)

190. Gao, W., Chen, Y., Zhang, Y., Zhang, Q., Zhang, L.: Nanoparticle-based local antimicrobial drug delivery. Adv. Drug. Deliv. Rev. 127, 46-57 (2018)

191. Raza, A., Sime, F.B., Cabot, P.J., Maqbool, F., Roberts, J.A., Falconer, J.R.: Solid nanoparticles for oral antimicrobial drug delivery: a review. Drug Discov. Today. 24(3), 858-866 (2019)

192. Jiao, Z., Zhang, B., Li, C., Kuang, W., Zhang, J., Xiong, Y., et al.: Carboxymethyl cellulose-grafted graphene oxide for efficient antitumor drug delivery. Nanotechnol. Rev. 7(4), 291-301 (2018)

193. Javanbakht, S., Namazi, H.: Doxorubicin loaded carboxymethyl cellulose/graphene quantum dot nanocomposite hydrogel films as a potential anticancer drug delivery system. Mater. Sci. Eng. C. 87, 50-59 (2018)

194. Dong, H., Jin, M., Liu, Z., Xiong, H., Qiu, X., Zhang, W., et al.: In vitro and in vivo brain-targeting chemo-photothermal therapy using graphene oxide conjugated with transferrin for Gliomas. Lasers Med. Sci. 31, 1123-1131 (2016)

195. Kazempour, M., Namazi, H., Akbarzadeh, A., Kabiri, R.: Synthesis and characterization of PEG-functionalized graphene oxide as an effective $\mathrm{pH}$-sensitive drug carrier. Artif. Cells. Nanomed. Biotechnol. 47(1), 90-94 (2019)

196. Chai, D., Hao, B., Hu, R., Zhang, F., Yan, J., Sun, Y., et al.: Delivery of oridonin and methotrexate via PEGylated graphene oxide. ACS Appl. Mater. Interfaces. 11(26), 22915-22924 (2019)

197. Borandeh, S., Abdolmaleki, A., Abolmaali, S.S., Tamaddon, A.M.: Synthesis, structural and in-vitro characterization of $\beta$-cyclodextrin grafted L-phenylalanine functionalized graphene oxide nanocomposite: a versatile nanocarrier for $\mathrm{pH}$-sensitive doxorubicin delivery. Carbohydr. Polym. 201, 151-161 (2018)
198. Prabakaran, S., Jeyaraj, M., Nagaraj, A., Sadasivuni, K.K., Rajan, M.: Polymethyl methacrylate-ovalbumin @ graphene oxide drug carrier system for high anti-proliferative cancer drug delivery. Appl. Nanosci. 9, 1487-1500 (2019)

199. Pourjavadi, A., Kohestanian, M., Yaghoubi, M.: Poly(glycidyl methacrylate)-coated magnetic graphene oxide as a highly efficient nanocarrier: preparation, characterization, and targeted DOX delivery. New J. Chem. 43(47), 18647-18656 (2019)

200. Deb, A., Vimala, R.: Camptothecin loaded graphene oxide nanoparticle functionalized with polyethylene glycol and folic acid for anticancer drug delivery. J. Drug Deliv. Sci. Technol. 43, 333-342 (2018)

201. Pourjavadi, A., Asgari, S., Hosseini, S.H.: Graphene oxide functionalized with oxygen-rich polymers as a $\mathrm{pH}$-sensitive carrier for co-delivery of hydrophobic and hydrophilic drugs. J. Drug Deliv. Sci. Technol. 56, 101542 (2020)

202. Pei, X., Zhu, Z., Gan, Z., Chen, J., Zhang, X., Cheng, X., et al.: PEGylated nano-graphene oxide as a nanocarrier for delivering mixed anticancer drugs to improve anticancer activity. Sci. Rep. 10, 1-15 (2020)

203. Gao, Y., Wu, J., Ren, X., Tan, X., Hayat, T., Alsaedi, A., et al.: Impact of graphene oxide on the antibacterial activity of antibiotics against bacteria. Environ. Sci. Nano. 4(5), 1016-1024 (2017)

204. Katuwavila, N.P., Amarasekara, Y., Jayaweera, V., Rajaphaksha, C., Gunasekara, C., Perera, I.C., et al.: Graphene oxide-based nanocomposite for sustained release of cephalexin. J. Pharm. Sci. 109(2), 1130-1135 (2020)

205. Saifullah, B., Chrzastek, A., Maitra, A., Naeemullah, B., Fakurazi, S., Bhakta, S., et al.: Novel anti-tuberculosis nanodelivery formulation of ethambutol with graphene oxide. Molecules 22(10), 1560 (2017)

206. Karimzadeh, Z., Javanbakht, S., Namazi, H.: Carboxymethylcellulose/MOF-5/Graphene oxide bio-nanocomposite as antibacterial drug nanocarrier agent. Bioimpacts. 9(1), 5-13 (2018)

207. Yang, D., Feng, L., Dougherty, C.A., Luker, K.E., Chen, D., Cauble, M.A., et al.: In vivo targeting of metastatic breast cancer via tumor vasculature-specific nano-graphene oxide. Biomaterials 104, 361-371 (2016)

208. Bullo, S., Buskaran, K., Baby, R., Dorniani, D., Fakurazi, S., Hussein, M.Z.: Dual drugs anticancer nanoformulation using graphene oxide-PEG as nanocarrier for protocatechuic acid and chlorogenic acid. Pharm. Res. 36, 1-11 (2019)

209. Javanbakht, S., Shaabani, A.: Encapsulation of graphene quantum dot-crosslinked chitosan by carboxymethylcellulose hydrogel beads as a pH-responsive bio-nanocomposite for the oral delivery agent. Int. J. Biol. Macromol. 123, 389-397 (2019)

210. Şenel, B., Demir, N., Büyükköroğlu, G., Yıldız, M.: Graphene quantum dots: synthesis, characterization, cell viability, genotoxicity for biomedical applications. Saudi Pharm. J. 27(6), 846-858 (2019)

211. Bayoumy, A.M., Refaat, A., Yahia, I.S., Zahran, H.Y., Elhaes, H., Ibrahim, M.A., et al.: Functionalization of graphene quantum dots (GQDs) with chitosan biopolymer for biophysical applications. Opt. Quantum. Electron. 52, 1-14 (2019)

212. Wei, Z., Yin, X., Cai, Y., Xu, W., Song, C., Wang, Y., et al.: Antitumor effect of a Pt-loaded nanocomposite based on graphene quantum dots combats hypoxia-induced chemoresistance of oral squamous cell carcinoma. Int. J. Nanomed. 13, 1505-1524 (2018)

213. De, S., Patra, K., Ghosh, D., Dutta, K., Dey, A., Sarkar, G., et al.: Tailoring the efficacy of multifunctional biopolymeric graphene oxide quantum dot-based nanomaterial as nanocargo in cancer therapeutic application. ACS Biomater. Sci. Eng. 4(2), 514-531 (2018)

214. Ko, N.R., Hong, S.H., Nafiujjaman, M., An, S.Y., Revuri, V., Lee, S.J., et al.: Glutathione-responsive PEGylated GQD-based 
nanomaterials for diagnosis and treatment of breast cancer. J. Ind. Eng. Chem. 71, 301-307 (2019)

215. Flak, D., Przysiecka, Ł, Nowaczyk, G., Scheibe, B., Kościński, M., Jesionowski, T., et al.: GQDs-MSNs nanocomposite nanoparticles for simultaneous intracellular drug delivery and fluorescent imaging. J. Nanoparticle. Res. 20, 1-19 (2018)

216. Yang, C., Chan, K.K., Xu, G., Yin, M., Lin, G., Wang, X., et al.: Biodegradable polymer-coated multifunctional graphene quantum dots for light-triggered synergetic therapy of pancreatic cancer. ACS Appl. Mater. Interfaces. 11(3), 2768-2781 (2018)

217. Rakhshaei, R., Namazi, H., Hamishehkar, H., Rahimi, M.: Graphene quantum dot cross-linked carboxymethyl cellulose nanocomposite hydrogel for $\mathrm{pH}$-sensitive oral anticancer drug delivery with potential bioimaging properties. Int. J. Biol. Macromol. 150, 1121-1129 (2020)

218. Sui, X., Luo, C., Wang, C., Zhang, F., Zhang, J., Guo, S.: Graphene quantum dots enhance anticancer activity of cisplatin via increasing its cellular and nuclear uptake. Nanomedicine 12(7), 1997-2006 (2016)

219. Sheng, Y., Dai, W., Gao, J., Li, H., Tan, W., Wang, J., et al.: $\mathrm{pH}$-sensitive drug delivery based on chitosan wrapped graphene quantum dots with enhanced fluorescent stability. Mater. Sci. Eng. C. 112, 110888 (2020)

220. Ko, N.R., Nafiujjaman, M., Lee, J.S., Lim, H.-N., Lee, Y.-K., Kwon, I.K.: Graphene quantum dot-based theranostic agents for active targeting of breast cancer. RSC Adv. 7(19), 11420-11427 (2017)

221. Song, S., Shen, H., Wang, Y., Chu, X., Xie, J., Zhou, N., et al.: Biomedical application of graphene: from drug delivery, tumor therapy, to theranostics. Colloids Surf. B Biointerfaces. 185, 110596 (2020)

222. Dash, B.S., Jose, G., Lu, Y.-J., Chen, J.-P.: Functionalized reduced graphene oxide as a versatile tool for cancer therapy. Int. J. Mol. Sci. 22(6), 2989 (2021)

223. Nomura, S., Morimoto, Y., Tsujimoto, H., Arake, M., Harada, M., Saitoh, D., et al.: Highly reliable, targeted photothermal cancer therapy combined with thermal dosimetry using a nearinfrared absorbent. Sci. Rep. 10, 1-7 (2020)

224. Robinson, J.T., Tabakman, S.M., Liang, Y., Wang, H., Casalongue, H.S., Vinh, D., et al.: Ultrasmall reduced graphene oxide with high near-infrared absorbance for photothermal therapy. J. Am. Chem. Soc. 133(17), 6825-6831 (2011)

225. Otari, S.V., Kumar, M., Anwar, M.Z., Thorat, N.D., Patel, S.K.S., Lee, D., et al.: Rapid synthesis and decoration of reduced graphene oxide with gold nanoparticles by thermostable peptides for memory device and photothermal applications. Sci. Rep. 7, 1-14 (2017)

226. Chen, X., Li, C., Wang, X., Zhao, X.: Infrared heating of reduced graphene oxide nanosheets as photothermal radiation therapeutic agents for tumor regressions. Mater. Res. Express. 6, 085080 (2019)

227. Zhang, D.-Y., Zheng, Y., Tan, C.-P., Sun, J.-H., Zhang, W., Ji, L.-N., et al.: Graphene oxide decorated with Ru(II)-polyethylene glycol complex for lysosome-targeted imaging and photodynamic/photothermal therapy. ACS Appl. Mater. Interfaces. 9(8), 6761-6771 (2017)

228. Jiang, W., Mo, F., Jin, X., Chen, L., Xu, L.J., Guo, L., et al.: Tumor-targeting photothermal heating-responsive nanoplatform based on reduced graphene oxide/mesoporous silica/hyaluronic acid nanocomposite for enhanced photodynamic therapy. Adv. Mater. Interfaces. 4(20), 1700425 (2017)

229. Shim, G., Kim, M.-G., Jin, H., Kim, J., Oh, Y.-K.: Claudin 4-targeted nanographene phototherapy using a Clostridium perfringens enterotoxin peptide-photosensitizer conjugate. Acta. Pharmacol. Sin. 38, 954-962 (2017)
230. Wang, L., Wang, M., Zhou, B., Zhou, F., Murray, C., Towner, R.A., et al.: PEGylated reduced-graphene oxide hybridized with Fe3O4 nanoparticles for cancer photothermal-immunotherapy. J. Mater. Chem. B. 7(46), 7406-7414 (2019)

231. Zhou, F., Wang, M., Luo, T., Qu, J., Chen, W.R.: Photo-activated chemo-immunotherapy for metastatic cancer using a synergistic graphene nanosystem. Biomaterials 265, 120421 (2021)

232. Yan, M., Liu, Y., Zhu, X., Wang, X., Liu, L., Sun, H., et al.: Nanoscale reduced graphene oxide-mediated photothermal therapy together with IDO inhibition and PD-L1 blockade synergistically promote antitumor immunity. ACS Appl. Mater. Interfaces. 11(2), 1876-1885 (2018)

233. Schroeder, K.L., Goreham, R.V., Nann, T.: Graphene quantum dots for theranostics and bioimaging. Pharm. Res. 33, 2337-2357 (2016)

234. Roy, S., Jaiswal, A.: Graphene-based nanomaterials for theranostic applications. Rep. Adv. Phys. Sci. 1(4), 1750011 (2018)

235. Dong, J., Wang, K., Sun, L., Sun, B., Yang, M., Chen, H., et al.: Application of graphene quantum dots for simultaneous fluorescence imaging and tumor-targeted drug delivery. Sens. Actuators B Chem. 256, 616-623 (2018)

236. Ju, J., Regmi, S., Fu, A., Lim, S., Liu, Q.: Graphene quantum dot based charge-reversal nanomaterial for nucleus-targeted drug delivery and efficiency controllable photodynamic therapy. J. Biophotonics 12(6), e201800367 (2019)

237. Sweetman, M.J., Hickey, S.M., Brooks, D.A., Hayball, J.D., Plush, S.E.: A practical guide to prepare and synthetically modify graphene quantum dots. Adv. Funct. Mater. 29(14), 1808740 (2019)

238. Feng, S., Pan, J., Li, C., Zheng, Y.: Folic acid-conjugated nitrogen-doped graphene quantum dots as a fluorescent diagnostic material for MCF-7 cells. Nanotechnology 31(13), 135701 (2020)

239. Costa, A.M., Silva, V.V.: Estratégias nanotecnológicas para diagnóstico e tratamento do câncer. Rev Saúde e Meio Ambient. 5(2), $1-13(2017)$

240. Matea, C.T., Mocan, T., Tabaran, F., Pop, T., Mosteanu, O., Puia, C., et al.: Quantum dots in imaging, drug delivery and sensor applications. Int. J. Nanomed. 12, 5421-5431 (2017)

241. Yao, X., Tian, Z., Liu, J., Zhu, Y., Hanagata, N.: Mesoporous silica nanoparticles capped with graphene quantum dots for potential chemo-photothermal synergistic cancer therapy. Langmuir 33(2), 591-599 (2016)

242. Hai, X., Feng, J., Chen, X., Wang, J.: Tuning the optical properties of graphene quantum dots for biosensing and bioimaging. J. Mater. Chem. B. 6(20), 3219-3234 (2018)

243. Li, L., Wu, G., Yang, G., Peng, J., Zhao, J., Zhu, J.-J.: Focusing on luminescent graphene quantum dots: current status and future perspectives. Nanoscale 5(20), 4015-4039 (2013)

244. Eda, G., Lin, Y.-Y., Mattevi, C., Yamaguchi, H., Chen, H.-A., Chen, I.-S., et al.: Blue photoluminescence from chemically derived graphene oxide. Adv. Mater. 22(4), 505-509 (2010)

245. Biswas, M.C., Islam, M.T., Nandy, P.K., Hossain, M.M.: Graphene quantum dots (GQDs) for bioimaging and drug delivery applications: a review. ACS Mater. Lett. 3(6), 889-911 (2021)

246. Sk, M.A., Ananthanarayanan, A., Huang, L., Lim, K.H., Chen, P.: Revealing the tunable photoluminescence properties of graphene quantum dots. J. Mater. Chem. C. 2(34), 6954-6960 (2014)

247. Hasan, M.T., Gonzalez-Rodriguez, R., Lin, C.-W., Campbell, E., Vasireddy, S., Tsedev, U., et al.: Rare-earth metal ions doped graphene quantum dots for near-IR in vitro/in vivo/ex vivo imaging applications. Adv. Opt. Mater. 8(21), 2000897 (2020)

248. Younis, M.R., He, G., Lin, J., Huang, P.: Recent advances on graphene quantum dots for bioimaging applications. Front. Chem. 8, 424 (2020) 
249. Wang, H., Mu, Q., Wang, K., Revia, R.A., Yen, C., Gu, X., et al.: Nitrogen and boron dual-doped graphene quantum dots for near-infrared second window imaging and photothermal therapy. Appl. Mater. Today. 14, 108-117 (2019)

250. Hasanzadeh, M., Shadjou, N.: What are the reasons for low use of graphene quantum dots in immunosensing of cancer biomarkers? Mater. Sci. Eng. C. 71, 1313-1326 (2017)

251. Yukawa, H., YBaba, Y.: In vivo fluorescence imaging and the diagnosis of stem cells using quantum dots for regenerative medicine. Anal. Chem. 89(5), 2671-2681 (2017)

252. Badrigilan, S., Shaabani, B., Aghaji, N.G., Mesbahi, A.: Graphene quantum dots-coated bismuth nanoparticles for improved CT imaging and photothermal performance. Int. J. Nanosci. 19(1), 1850043 (2018)

253. Wang, K.-Q., Li, K.-R., Yu, B., Shen, Y.-Q., Cong, H.-L.: Application of PEGylated graphene quantum dots in cell imaging. Ferroelectrics 547(1), 21-26 (2019)

254. Feng, S., Li, H., Pan, J., Li, C., Zheng, Y.: Preparation and cell imaging of nitrogen-doped graphene quantum dot conjugated indomethacin. IOP Conf. Ser. Earth. Environ. Sci. 358, 032006 (2019)

255. Campbell, E., Hasan, M.T., Rodriguez, R.G., Akkaraju, G.R., Naumov, A.V.: Doped graphene quantum dots for intracellular multicolor imaging and cancer detection. ACS Biomater. Sci. Eng. 5(9), 4671-4682 (2019)

256. Su, X., Chan, C., Shi, J., Tsang, M.K., Pan, Y., Cheng, C., et al.: A graphene quantum dot@ $\mathrm{Fe} 3 \mathrm{O} 4 @ \mathrm{SiO} 2$ based nanoprobe for drug delivery sensing and dual-modal fluorescence and MRI imaging in cancer cells. Biosens. Bioelectron. 92, 489-495 (2017)

257. Wang, Y., Song, H., Wang, G., Yang, X., Wang, J., Wei, H.: 131I-labeled PEG and folic acid co-functionalized graphene quantum dots for tumor-targeted imaging. J. Radioanal. Nucl. Chem. 319, 1119-1125 (2019)

258. Nasrollahi, F., Sana, B., Paramelle, D., Ahadian, S., Khademhosseini, A., Lim, S.: Incorporation of graphene quantum dots, iron, and doxorubicin in/on ferritin nanocages for bimodal imaging and drug delivery. Adv. Ther. 3(3), 1900183 (2020)

259. Zhang, H., Ba, S., Yang, Z., Wang, T., Lee, J.Y., Li, T., Shao, F.: Graphene quantum dot-based nanocomposites for diagnosing cancer biomarker APE1 in living cells. ACS Appl. Mater. Interfaces. 12(12), 13634-13643 (2020)

260. Javanbakht, S., Pooresmaeil, M., Namazi, H.: Green one-pot synthesis of carboxymethylcellulose/Zn-based metal-organic framework/graphene oxide bio-nanocomposite as a nanocarrier for drug delivery system. Carbohydr. Polym. 208, 294-301 (2019)

261. Prasad, R., Yadav, A.S., Gorain, M., Chauhan, D.S., Kundu, G.C., Srivastava, R., et al.: Graphene oxide supported liposomes as red emissive theranostics for phototriggered tissue visualization and tumor regression. ACS Appl. Bio. Mater. 2(8), 3312-3320 (2019)

262. Xu, Y., Hu, X., Guan, P., Du, C., Tian, Y., Ding, S., et al.: A novel controllable molecularly imprinted drug delivery system based on the photothermal effect of graphene oxide quantum dots. J. Mater. Sci. 54, 9124-9139 (2019)

263. Badrigilan, S., Shaabani, B., Gharehaghaji, N., Mesbahi, A.: Iron oxide/bismuth oxide nanocomposites coated by graphene quantum dots: "Three-in-one" theranostic agents for simultaneous CT/MR imaging-guided in vitro photothermal therapy. Photodiagn Photodyn. Ther. 25, 504-514 (2019)

264. Chang, X., Zhang, Y., Xu, P., Zhang, M., Wu, H., Yang, S.: Graphene oxide / MnWO4 nanocomposite for magnetic resonance / photoacoustic dual-model imaging and tumor photothermo-chemotherapy. Carbon N Y. 138, 397-409 (2018)
265. Qian, R., Maiti, D., Zhong, J., Xiong, S.S., Zhou, H., Zhu, R., et al.: Multifunctional nano-graphene based nanocomposites for multimodal imaging guided combined radioisotope therapy and chemotherapy. Carbon N Y. 149, 55-62 (2019)

266. Gonzalez-Rodriguez, R., Campbell, E., Naumov, A.: Multifunctional graphene oxide/iron oxide nanoparticles for magnetic targeted drug delivery dual magnetic resonance/fluorescence imaging and cancer sensing. PLoS ONE 14(6), 217072 (2019)

267. Zhang, B., Wang, Y., Zhai, G.: Biomedical applications of the graphene-based materials. Mater. Sci. Eng. C Mater. Biol. Appl. 61(9), 53-64 (2016)

268. Usman, M.S., Hussein, M.Z., Fakurazi, S., Masarudin, M.J., Saad, F.F.A.: A bimodal theranostic nanodelivery system based on [graphene oxide-chlorogenic acid-gadolinium/gold] nanoparticles. PLoS ONE 13(7), e0200760 (2018)

269. Bansal, S., Singh, J., Kumari, U., Kaur, I.P., Barnwal, R.P., Kumar, R., et al.: Development of biosurfactant-based graphene quantum dot conjugate as a novel and fluorescent theranostic tool for cancer. Int. J. Nanomed. 14, 809-818 (2019)

270. Nasrollahi, F., Koh, Y.R., Chen, P., Varshosaz, J., Khodadadi, A.A., Lim, S.: Targeting graphene quantum dots to epidermal growth factor receptor for delivery of cisplatin and cellular imaging. Mater. Sci. Eng. C 94, 247-257 (2019)

271. Yuan, J., Gao, H., Sui, J., Duan, H., Chen, W.N., Ching, C.B.: Cytotoxicity evaluation of oxidized single-walled carbon nanotubes and graphene oxide on human hepatoma HepG2 cells: an iTRAQ-coupled 2D LC-MS/MS proteome analysis. Toxicol. Sci. 126(1), 149-161 (2012)

272. Gurunathan, S., Han, J.W., Abdal Daye, A., Eppakayala, V., Kim, J.: Oxidative stress-mediated antibacterial activity of graphene oxide and reduced graphene oxide in Pseudomonas aeruginosa. Int. J. Nanomed. 7, 5901-5914 (2012)

273. Horváth, L., Magrez, A., Burghard, M., Kern, K., Forró, L., Schwaller, B.: Evaluation of the toxicity of graphene derivatives on cells of the lung luminal surface. Carbon N Y. 64, 45-60 (2013)

274. Lu, X., Feng, X., Werber, J.R., Chu, C., Zucker, I., Kim, J.-H., et al.: Enhanced antibacterial activity through the controlled alignment of graphene oxide nanosheets. Proc. Natl. Acad. Sci. 114(46), 9793-9801 (2017)

275. Diiulio, M., Zappacosta, R., Diodovico, S., Campli, E., Diiani, G., Fontana, A., et al.: Antimicrobial and antibiofilm efficacy of graphene oxide against chronic wound microorganisms. Antimicrob. Agents. Chemother. 62(7), e00547-e618 (2018)

276. Wei, C., Liu, Z., Jiang, F., Zeng, B., Huang, M., Yu, D.: Cellular behaviours of bone marrow-derived mesenchymal stem cells towards pristine graphene oxide nanosheets. Cell Prolif. 50, 1-10 (2017)

277. Kumar, S., Raj, S., Kolanthai, E., Sood, A.K., Sampath, S., Chatterjee, K.: Chemical functionalization of graphene to augment stem cell osteogenesis and inhibit biofilm formation on polymer composites for orthopedic applications. ACS Appl. Mater. Interfaces. 7(5), 3237-3252 (2015)

278. Zhou, M., Lozano, N., Wychowaniec, J.K., Hodgkinson, T., Richardson, S.M., Kostarelos, K., et al.: Graphene oxide: a growth factor delivery carrier to enhance chondrogenic differentiation of human mesenchymal stem cells in 3D hydrogels. Acta Biomater. 96, 271-280 (2019)

279. Jiao, D., Wang, J., Yu, W., Zhang, N., Zhang, K., Bai, Y.: Gelatin reduced graphene oxide nanosheets as kartogenin nanocarrier induces rat ADSCs chondrogenic differentiation combining with autophagy modification. Materials 14(5), 1053 (2021)

280. Lv, M., Zhang, Y., Liang, L., Wei, M., Hu, W., Li, X., et al.: Effect of graphene oxide on undifferentiated and retinoic aciddifferentiated SH-SY5Y cells line. Nanoscale 4(13), 3861-3866 (2012) 
281. Khim Chng, E.L., Chua, C.K., Pumera, M.: Graphene oxide nanoribbons exhibit significantly greater toxicity than graphene oxide nanoplatelets. Nanoscale 6(18), 10792-10797 (2014)

282. Akhavan, O., Ghaderi, E., Emamy, H., Akhavan, F.: Genotoxicity of graphene nanoribbons in human mesenchymal stem cells. Carbon N Y. 54, 419-431 (2013)

283. Talukdar, Y., Rashkow, J.T., Lalwani, G., Kanakia, S., Sitharaman, B.: The effects of graphene nanostructures on mesenchymal stem cells. Biomaterials 35(18), 4863-4877 (2014)

284. Mullick Chowdhury, S., Lalwani, G., Zhang, K., Yang, J.Y., Neville, K., Sitharaman, B.: Cell specific cytotoxicity and uptake of graphene nanoribbons. Biomaterials 34(1), 283-293 (2013)

285. Chng, E.L.K., Sofer, Z., Pumera, M.: Cytotoxicity profile of highly hydrogenated graphene. Chemistry 20(21), 6366-6373 (2014)

286. Yuan, X., Liu, Z., Guo, Z., Ji, Y., Jin, M., Wang, X.: Cellular distribution and cytotoxicity of graphene quantum dots with different functional groups. Nanoscale Res. Lett. 9, 1-9 (2014)

287. Chong, Y., Ma, Y., Shen, H., Tu, X., Zhou, X., Xu, J., et al.: The in vitro and in vivo toxicity of graphene quantum dots. Biomaterials 35(19), 5041-5048 (2014)

288. Chen, J., Yang, L., Li, S., Ding, W.: Various physiological response to graphene oxide and amine-functionalized graphene oxide in wheat (Triticum aestivum). Molecules 23(5), 1104 (2018)

289. Salesa, B., Serrano-Aroca, Á.: Multi-layer graphene oxide in human keratinocytes: time-dependent cytotoxicity, proliferation, and gene expression. Coatings (Basel). 11(4), 414 (2021)

290. Audira, G., Lee, J.S., Siregar, P., Malhotra, N., Rolden, M.J.M., Huang, J.C., et al.: Comparison of the chronic toxicities of graphene and graphene oxide toward adult zebrafish by using biochemical and phenomic approaches. Environ. Pollut. 278, 116907 (2021)

291. Cao, Z., Su, M., Wang, H., Zhou, L., Meng, Z., Xiong, G., et al.: Carboxyl graphene oxide nanoparticles induce neurodevelopmental defects and locomotor disorders in zebrafish larvae. Chemosphere 270, 128611 (2021)

292. Chen, Z., Yu, C., Khan, I.A., Tang, Y., Liu, S., Yang, M.: Toxic effects of different-sized graphene oxide particles on zebrafish embryonic development. Ecotoxicol. Environ. Saf. 197, 110608 (2020)

293. Damora, M., Lamberti, A., Fontana, M., Giordani, S.: Toxicity assessment of laser-induced graphene by zebrafish during development. J. Phys. Mater. 3, 34008 (2020)

294. Manjunatha, B., Seo, E., Park, S.H., Kundapur, R.R., Lee, S.J.: Pristine graphene and graphene oxide induce multi-organ defects in zebrafish (Danio rerio) larvae/juvenile: an in vivo study. Environ. Sci. Pollut. Res. 28, 34664-34675 (2021)

295. Zou, W., Zhang, X., Ouyang, S., Hu, X., Zhou, Q.: Graphene oxide nanosheets mitigate the developmental toxicity of TDCIPP in zebrafish via activating the mitochondrial respiratory chain and energy metabolism. Sci. Total Environ. 727, 138486 (2020)

296. Cellot, G., Vranic, S., Shin, Y., Worsley, R., Rodrigues, A.F., Bussy, C., et al.: Graphene oxide nanosheets modulate spinal glutamatergic transmission and modify locomotor behaviour in an in vivo zebrafish model. Nanoscale Horiz. 5(8), 1250-1263 (2020)

297. Dziewiecka, M., Karpeta-Kaczmarek, J., Augustyniak, M., Majchrzycki, Ł, Augustyniak-Jabłokow, M.A.: Evaluation of in vivo graphene oxide toxicity for Acheta domesticus in relation to nanomaterial purity and time passed from the exposure. J. Hazard. Mater. 305, 30-40 (2016)

298. Dziewięcka, M., Flasz, B., Rost-Roszkowska, M., Kędziorski, A., Kochanowicz, A., Augustyniak, M.: Graphene oxide as a new anthropogenic stress factor-multigenerational study at the molecular, cellular, individual and population level of Acheta domesticus. J. Hazard. Mater. 396, 122775 (2020)

299. Hrynko, I., Łozowicka, B., Kaczyński, P.: Development of precise micro analytical tool to identify potential insecticide hazards to bees in guttation fluid using LC-ESI-MS/MS. Chemosphere 263, 128143 (2021)

300. Fang, Y., Lu, Z., Li, M., Qu, J., Ye, W., Li, F., et al.: An assessment of the reproductive toxicity of GONPs exposure to Bombyx mori. Ecotoxicol. Environ. Saf. 210, 111888 (2021)

301. Benelli, G.: Mode of action of nanoparticles against insects. Environ. Sci. Pollut. Res. 25, 12329-12341 (2018)

302. Wang, X., Xie, H., Wang, Z., He, K., Jing, D.: Graphene oxide as a multifunctional synergist of insecticides against lepidopteran insect. Environ. Sci. Nano. 6(1), 75-84 (2019)

303. Ling, F., Hong-bo, L., Yan-hong, Z., Bo, L.: Technology research on oxidative degree of graphite oxide prepared by hummers method. Carbon N Y. 124, 10-14 (2005)

304. Shen, J., Hu, Y., Shi, M., Lu, X., Qin, C., Li, C., et al.: Fast and facile preparation of graphene oxide and reduced graphene oxide nanoplatelets. Chem. Mater. 21(15), 3514-3520 (2009)

305. Su, C.-Y., Xu, Y., Zhang, W., Zhao, J., Tang, X., Tsai, C.-H., et al.: Electrical and spectroscopic characterizations of ultralarge reduced graphene oxide monolayers. Chem. Mater. 21(23), 5674-5680 (2009)

306. Eigler, S., Enzelberger-Heim, M., Grimm, S., Hofmann, P., Kroener, W., Geworski, A., et al.: Wet chemical synthesis of graphene. Adv. Mater. 25(26), 3583-3587 (2013)

307. Panwar, V., Chattree, A., Pal, K.: A new facile route for synthesizing of graphene oxide using mixture of sulfuric-nitric-phosphoric acids as intercalating agent. Phys E Low Dimens. Syst. Nanostruct. 73, 235-241 (2015)

308. Peng, L., Xu, Z., Liu, Z., Wei, Y., Sun, H., Li, Z., et al.: An ironbased green approach to 1 -h production of single-layer graphene oxide. Nat. Commun. 6, 1-9 (2015)

309. Rosillo-Lopez, M., Salzmann, C.G.: A simple and mild chemical oxidation route to high-purity nano-graphene oxide. Carbon $\mathrm{N}$ Y. 106, 56-63 (2016)

310. Dimiev, A.M., Ceriotti, G., Metzger, A., Kim, N.D., Tour, J.M.: Chemical mass production of graphene nanoplatelets in $\sim 100 \%$ yield. ACS Nano 10(1), 274-279 (2015)

311. Pei, S., Wei, Q., Huang, K., Cheng, H.-M., Ren, W.: Green synthesis of graphene oxide by seconds timescale water electrolytic oxidation. Nat. Commun. 9, 1-9 (2018)

312. Ranjan, P., Agrawal, S., Sinha, A., Rao, T.R., Balakrishnan, J., Thakur, A.D.: A low-cost non-explosive synthesis of graphene oxide for scalable applications. Sci. Rep. 8, 1-13 (2018)

313. Tian, J., Wu, S., Yin, X., Wu, W.: Novel preparation of hydrophilic graphene/graphene oxide nanosheets for supercapacitor electrode. Appl. Surf. Sci. 496, 143696 (2019)

314. Hou, Y., Lv, S., Liu, L., Liu, X.: High-quality preparation of graphene oxide via the Hummers' method: Understanding the roles of the intercalator, oxidant, and graphite particle size. Ceram. Int. 46(2), 2392-2402 (2020)

315. Silva, L.M.C., Gonçalves, B.S., Braga, J.O., Souza, T.C., Castro, V.G., Silva, G.G., et al.: Preparation of titania-reduced graphene oxide composite coatings with electro- and photosensitive properties. Appl. Surf. Sci. 538, 148029 (2021)

316. Sun, L.: Structure and synthesis of graphene oxide. Chinese J. Chem. Eng. 27(10), 2251-2260 (2019)

317. Yang, L., Xue, J., Jia, Y., Zhang, Y., Wu, D., Ma, H., et al.: Construction of well-ordered electrochemiluminescence sensing interface using peptide-based specific antibody immobilizer and $N$-(aminobutyl)- $N$-(ethylisoluminol) functionalized ferritin as signal indicator for procalcitonin analysis. Biosens. Bioelectron. 142, 111562 (2019) 
318. Sun, Y., Zhou, T., Li, W., Yu, F., Ma, J.: Amino-functionalized alginate/graphene double-network hydrogel beads for emerging contaminant removal from aqueous solution. Chemosphere 241, 125110 (2020)

319. Ahmed, M.S., Kim, Y.-B.: 3D graphene preparation via covalent amide functionalization for efficient metal-free electrocatalysis in oxygen reduction. Sci. Rep. 7, 1-13 (2017)

320. Fan, F., Zhang, B., Cao, Y., Yang, X., Gu, J., Chen, Y.: Conjugated polymer covalently modified graphene oxide quantum dots for ternary electronic memory devices. Nanoscale 9(30), 10610-10618 (2017)

321. Yu, B., Wang, J., Yang, X., Wang, W., Cai, X.: Preparation of polyglycerol mediated superparamagnetic graphene oxide nanocomposite and evaluation of its adsorption properties on tetracycline. Environ. Sci. Pollut. Res. 26, 32345-32359 (2019)

322. Yao, B., Li, C., Ma, J., Shi, G.: Porphyrin-based graphene oxide frameworks with ultra-large d-spacings for the electrocatalyzation of oxygen reduction reaction. Phys. Chem. Chem. Phys. 17(29), 19538-19545 (2015)

323. Luceño-Sánchez, J.A., Díez-Pascual, A.M.: Grafting of polypyrrole-3-carboxylic acid to the surface of hexamethylene diisocyanate-functionalized graphene oxide. Nanomaterials 9(8), 1095 (2019)

324. Tachi, S., Morita, H., Takahashi, M., Okabayashi, Y., Hosokai, T., Sugai, T., et al.: Quantum yield enhancement in graphene quantum dots via esterification with benzyl alcohol. Sci. Reports. 9, 1-7 (2019)

325. Li, Y., Li, J., Li, Y., Li, Y., Song, Y., Niu, S., et al.: Ultrasonicassisted preparation of graphene oxide carboxylic acid polyvinyl alcohol polymer film and studies of thermal stability and surface resistivity. Ultrason. Sonochem. 40, 798-807 (2018)

326. Lee, C.Y., Le, Q.V., Kim, C., Kim, S.Y.: Use of silane-functionalized graphene oxide in organic photovoltaic cells and organic light-emitting diodes. Phys. Chem. Chem. Phys. 17(14), 93699374 (2015)
327. Zhu, L., Liu, Y., Peng, X., Li, Y., Men, Y.-L., Liu, P., et al.: Noble-metal-free CdS nanoparticle-coated graphene oxide nanosheets favoring electron transfer for efficient photoreduction of CO2. ACS Appl. Mater. Interfaces. 12(11), 12892-12900 (2020)

328. Nuin, E., Lloret, V., Amsharov, K., Hauke, F., Abellán, G., Hirsch, A.: Isomerically pure star-shaped triphenylene-perylene hybrids involving highly extended $\pi$-conjugation. Chemistry 24(18), 4671-4679 (2018)

329. Cho, J., Jeon, I., Kim, S.Y., Lim, S., Jho, J.Y.: Improving dispersion and barrier properties of polyketone/graphene nanoplatelet composites via noncovalent functionalization using aminopyrene. ACS Appl. Mater. Interfaces. 9(33), 27984-27994 (2017)

330. Ni, H., Xu, F., Tomsia, A.P., Saiz, E., Jiang, L., Cheng, Q.: Robust bioinspired graphene film via $\pi-\pi$ cross-linking. ACS Appl. Mater. Interfaces. 9(29), 24987-24992 (2017)

331. Han, S.G., Kim, J.K., Shin, J.H., Hwang, J.H., Lee, J.S., Kim, T.G., et al.: Pulmonary responses of Sprague-Dawley rats in single inhalation exposure to graphene oxide nanomaterials. Biomed. Res. Int. 25, 1-9 (2015)

332. Liang, S., Xu, S., Zhang, D., He, J., Chu, M.: Reproductive toxicity of nanoscale graphene oxide in male mice. Nanotoxicology 9(1), 92-105 (2015)

333. Sydlik, S.A., Jhunjhunwala, S., Webber, M.J., Anderson, D.G., Langer, R.: In vivo compatibility of graphene oxide with differing Oxidation states. ACS Nano 9(4), 3866-3874 (2015)

334. Yang, K., Gong, H., Shi, X., Wan, J., Zhang, Y., Liu, Z.: In vivo biodistribution and toxicology of functionalized nano-graphene oxide in mice after oral and intraperitoneal administration. Biomaterials 34(11), 2787-2795 (2013)

Publisher's Note Springer Nature remains neutral with regard to jurisdictional claims in published maps and institutional affiliations.

\section{Authors and Affiliations}

Tais Monteiro Magne ${ }^{1} \cdot$ Thamires de Oliveira Vieira $^{1} \cdot$ Luciana Magalhães Rebelo Alencar $^{2} \cdot$
Francisco Franciné Maia Junior $^{3} \cdot$ Sara Gemini-Piperni $^{4} \cdot$ Samuel V. Carneiro $^{5} \cdot$ Lillian M. U. D. Fechine $^{5} \cdot$
Rafael M. Freire $^{6} \cdot$ Kirill Golokhvast $^{7,8} \cdot$ Pierangelo Metrangolo $^{9} \cdot$ Pierre B. A. Fechine $^{5} \cdot$ Ralph Santos-Oliveira $^{1,10}$

Ralph Santos-Oliveira

presidenciaradiofarmacia@gmail.com; roliveira@ien.gov.br

1 Brazilian Nuclear Energy Commission, Nuclear Engineering Institute, Rio de Janeiro 21941906, Brazil

2 Biophysics and Nanosystems Laboratory, Department of Physics, Federal University of Maranhão, São Luis, Maranhão 65080805, Brazil

3 Department of Natural Sciences, Mathematics and Statistics, Federal Rural University of the Semi-Arid, Mossoró, RN 59625-900, Brazil

4 Laboratory of Advanced Science, Universidade Unigranrio, Duque de Caxias, RJ 25071-202, Brazil

5 Group of Chemistry of Advanced Materials (GQMat)-Department of Analytical Chemistry and Physic-Chemistry, Federal University of Ceará-Campus do Pici, Fortaleza, Ceará 60451-970, Brazil
6 Institute of Applied Chemical Sciences, Universidad Autónoma de Chile, 8910060 Santiago, Chile

7 Education and Scientific Center of Nanotechnology, School of Engineering, Far Eastern Federal University, Vladivostok, Russia

8 N.I. Vavilov All-Russian Institute of Plant Genetic Resources, Saint-Petersburg, Russia

9 Laboratory of Supramolecular and Bio-Nanomaterials, Department of Chemistry, Materials, and Chemical Engineering "Giulio Natta" Politecnico Di Milano, Via L. Mancinelli 7, 20131 Milano, Italy

10 Laboratory of Nanoradiopharmacy and Synthesis of Radiopharmaceuticals, Zona Oeste State University, Av Manuel Caldeira de Alvarenga, 200, Campo Grande, Rio de Janeiro 2100000, Brazil 